\title{
Minorations des hauteurs normalisées des sous-variétés de variétés abeliennes II
}

\author{
Sinnou David et Patrice Philippon
}

\begin{abstract}
Résumé. E. UlLMO and S. ZhANG ont montré que les points de hauteur arbitrairement petite sur une sous-variété algébrique (non « dégénérée ») d'une variété abelienne (toutes deux définies sur un corps de nombres), ne peuvent être ZARISKI dense dans cette variété. Nous avons donné une autre preuve quantitative de ce résultat; dans le cas des tores, nous en avons ensuite donné une version totalement explicite. Ce travail a trouvé des applications, notamment en liaison avec le théorème du sous-espace. Nous consacrons donc ce texte à des minorations totalement explicites pour la hauteur normalisée, ainsi que pour les minimums successifs (de la hauteur normalisée) des sous-variétés de variétés abeliennes définies sur un corps de nombres. Ces minorations ont déjà trouvé une application dans un travail récent de G. RÉMOND, qui compte les variétés exceptionnelles dans les théorèmes de P. Vojta and G. Faltings (ex conjectures de Mordell et $\mathrm{S}$. LANG respectivement).
\end{abstract}

Abstract. E. UlLMO and S. ZHANG have shown that the points of arbitrarily small normalized heights on an algebraic subvariety of an abelian variety (defined over a number field), which is not of a special type, cannot be ZARISKI dense. We gave an alternative quantitative proof of this property that we made completely explicit in the analogous toric case. This latter work has found applications to diophantine geometry, especially in connection with the subspace theorem. The present paper is devoted to establishing completely explicit lower bounds for normalized heights and successive minima (of the normalized height) of algebraic subvarieties of abelian varieties defined over a number field. The results presented and proved here have found an application in the recent work of G. RÉMOND, counting exceptional varieties in the theorems of P. VOJTA and G. FALtings (ex MoRdelL's and LANG's conjectures respectively).

Mathematics Subject Classification (2000). 11G10, 11J81, 14G40.

Mots clés. Hauteur, variétés abeliennes, géométrie diophantienne, effectivité.

\section{Introduction et résultats}

Le problème maintenant classique, dit de BogOMOLOV, revient à demander si l'ensemble des points algébriques d'une sous-variété algébrique d'une variété abelienne, de hauteurs arbitrairement petites, peut être ZARISKI dense dans la variété. Cette question qualitative a été résolue (négativement, sauf dégénérescences évidentes) par S. ZHANG (confer [Zh2]) en s'appuyant sur les propriétés 
d'équirépartition de L. SzPiro, E. Ullmo et S. Zhang (confer [Sz-Ul-Zh]) et le travail d'E. UlLmo (confer [Ul]) concernant le cas des courbes plongées dans leur jacobienne. Nous avons aussi donné une démonstration alternative quantitative de cette même propriété dans [Da-Phi1], puis une version totalement explicite dans le cas des sous-variétés des tores multiplicatifs (confer [Da-Phi2]). L'intérêt pour les applications de ces résultats toriques étant réel, nous reprenons ici la démonstration de [Da-Phi1] pour donner dans le cas abelien des minorations également explicites de la hauteur normalisée et des minimums successifs de la hauteur normalisée des points d'une sous-variété algébrique d'une variété abelienne, définies sur $\overline{\mathbb{Q}}$. Les résultats présentés et établis ici trouvent une première application dans le travail récent de G. RÉMOND [Ré].

On supposera dorénavant que $A$ désigne une variété abelienne définie sur un corps de nombres $\boldsymbol{k}$ et munie d'un fibré ample et symétrique $\mathcal{M}$, qui permet de définir une notion de hauteur normalisée $\hat{h}:=\hat{h}_{\mathcal{M} \otimes 16}$ sur les sous-variétés algébriques de $A$ définies sur $\overline{\mathbb{Q}}$.

Soit $X$ une sous-variété algébrique de $A$ définie sur $\overline{\mathbb{Q}}$, on s'intéresse aux points de petites hauteurs de $X(\overline{\mathbb{Q}})$. Introduisons, comme dans [Da-Phi2], une série de minimums successifs pour $j=1, \ldots, \operatorname{dim}(X)$ :

$$
\hat{\mu}_{j}^{\circ}(X):=\sup _{Y} \inf \{\hat{h}(x) ; x \in(X \backslash Y)(\overline{\mathbb{Q}})\},
$$

où le supremum est pris sur les sous-ensembles algébriques $Y$ de $X$, définis sur $\overline{\mathbb{Q}}$, constitués de sous-variétés de $X$ de codimension $j$ dans $X$ et de translatées de sousvariétés abeliennes (non nécessairement par des points de torsion) contenues dans $X$, de codimension $<j$ dans $X$. En particulier, les $\hat{\mu}_{j}^{\circ}(X)$ minorent les minimums successifs de la hauteur normalisée sur le complémentaire $X^{\circ}$ dans $X$ de l'union des translatées (mais non nécessairement par des points de torsion) de sous-variétés abeliennes de A contenues dans $X$, de dimension $\geqslant 1$.

On remarquera que $\hat{\mu}_{1}^{\circ}(X)$ n'est autre que le minimum essentiel ${ }^{1}$ de $\hat{h}$ sur $X$, aussi noté $\hat{\mu}^{\text {ess }}(X)$, qui pour sa part, est généralement défini par :

$$
\hat{\mu}^{\text {ess }}(X):=\sup _{Y} \inf \{\hat{h}(x) ; x \in(X \backslash Y)(\overline{\mathbb{Q}})\},
$$

où le supremum est cette fois pris sur les sous-ensembles algébriques $Y$ de $X$ de codimension 1.

Par ailleurs, on a bien évidemment, par définition,

$$
\hat{\mu}_{1}^{\circ}(X) \geq \cdots \geq \hat{\mu}_{\operatorname{dim}(X)}^{\circ}(X) .
$$

Nous pouvons maintenant décrire les résultats que nous obtenons. Nous supposerons dans les énoncés qui suivent que la variété $A$ est principalement polarisée

\footnotetext{
${ }^{1}$ Sauf bien sûr dans le cas où $X$ est un translaté d'une sous-variété abelienne de $A$.
} 
par $\mathcal{M}$ et nous notons $g$ sa dimension. Nous désignerons par $h(A)$ la hauteur projective de l'origine de $A$ dans le plongement associé à $\mathcal{M}^{\otimes 16}$ (voir notation 3.2 ).

Théorème 1.1. Soit $X \subset A$ une sous-variété algébrique d'une variété abelienne, toutes deux définies sur $\overline{\mathbb{Q}}$, qui n'est pas translatée d'une sous-variété abelienne, alors

$$
(\operatorname{dim}(X)+1) \hat{\mu}^{\mathrm{ess}}(X) \geq \frac{\hat{h}(X)}{\operatorname{deg}(X)} \geq \frac{\min \left\{1 ; \mathcal{R}_{\text {inj }}\right\}^{2(b+1)}}{2^{9 g^{3}} \operatorname{deg}(X)^{2 k(b+1)}}
$$

où $k$ désigne le nombre minimal de copies de $X-X$ dont la somme est une sous-variété abelienne de A, b la dimension de cette sous-variété abelienne et $\mathcal{R}_{\text {inj }}$ désigne la plus petite norme de Riemann d'une période d'une conjuguée de A sur $\mathbb{Q}$ (voir la définition 4.4 pour plus de précisions).

On en déduit les théorèmes suivants sur le dernier des minimums successifs introduits précédemment. On se donne une sous-variété algébrique $X$ de $A$ définie sur $\overline{\mathbb{Q}}$ et on pose avec les notations du théorème 1.1 :

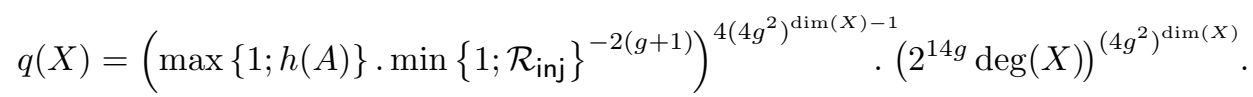

Théorème 1.2. Soit $X \subset A$ une sous-variété algébrique d'une variété abelienne toutes deux définies sur $\overline{\mathbb{Q}}$, les points $x \in X^{\circ}(\overline{\mathbb{Q}})$ satisfaisant $\hat{h}(x)<1 / q(X)$ sont en nombre fini majoré par $q(X)$. En particulier, $\hat{\mu}_{\operatorname{dim}(X)}^{\circ}(X) \geqslant 1 / q(X)$.

On dispose bien entendu également d'estimations explicites pour les minimums intermédiaires $\hat{\mu}_{j}^{0}(\cdot), 1 \leq j \leq \operatorname{dim}(X)-1$; le lecteur pourra se reporter au paragraphe 5 , théorème 5.3 pour les trouver.

On déduit du théorème 1.2 le résultat de décompte suivant :

Théorème 1.3. Soit $X \subset A$ une sous-variété algébrique d'une variété abelienne toutes deux définies sur $\overline{\mathbb{Q}}$ et $\Gamma \subset A(\overline{\mathbb{Q}})$ un sous-groupe de rang fini $r$. Pour tout nombre réel $a \geqslant 1$, il existe au plus $q(X)(5 a q(X))^{r / 2}$ points $x \in \Gamma \cap X^{\circ}(\overline{\mathbb{Q}})$ satisfaisant $\hat{h}(x) \leqslant a$.

On sait estimer (voir lemme 6.8) le rayon d'injectivité $\mathcal{R}_{\text {inj }}$ en termes de la hauteur $h(A)$ et du degré $d$ d'un corps $\boldsymbol{k}$ de définition de la variété abelienne polarisée $(A, \mathcal{M})$. On obtient ainsi :

Théorème 1.4. Soit $X \subset A$ une sous-variété algébrique d'une variété abelienne toutes deux définies sur $\overline{\mathbb{Q}}$, avec les notations introduites dans les énoncés précédents et en posant $h_{0}(A)=d \max \{1 ; h(A)\}$, où $d=[\boldsymbol{k}: \mathbb{Q}]$, on a

$$
q(X) \leq\left(2^{16 g} \cdot h_{0}(A)^{2 / g} \cdot \operatorname{deg}(X)\right)^{\left(4 g^{2}\right)^{\operatorname{dim}(X)},},
$$

et si $X$ n'est pas translatée d'une sous-variété abelienne :

$$
(\operatorname{dim}(X)+1) \hat{\mu}^{\text {ess }}(X) \geq \frac{\hat{h}(X)}{\operatorname{deg}(X)} \geq 2^{-11 g^{3}} \cdot \operatorname{deg}(X)^{-2 k(b+1)} \cdot h_{0}(A)^{-g-1} .
$$


Un des intérêts de ces résultats réside dans leur totale effectivité, et on notera qu'en dehors de la dimension et du degré de la variété algébrique considérée, les seuls paramètres qui interviennent sont la dimension de la variété abelienne ambiante (sa hauteur relative à un corps de définition pour les théorèmes 1.2 et 1.3) et son « rayon d'injectivité absolu », au sens de J.-B. Bost (confer [Bo]). En appendice, on minore explicitement cette dernière quantité en fonction de la dimension de la variété abelienne et de sa hauteur relative à un corps de définition dans le plongement considéré, ce qui conduit au théorème 1.4 ci-dessus. Les plongements projectifs des variétés abeliennes obtenus à l'aide des coordonnées de MUMFORD ( confer $[\mathrm{Mu}]$ ), qui généralisent les plongements thêta classiques des variétés abeliennes, sont l'outil essentiel qui permet de mener tous les calculs de façon explicite. En particulier, on écrit des formules de duplication et d'addition dans ces plongements et on en estime degrés, hauteurs et croissances. Ceci permet d'étendre les résultats de Y. MANIN et Y. ZARHIN ( confer [Ma-Za]) sur la constante de comparaison entre hauteurs projectives et hauteurs normalisées des points des variétés abeliennes aux sous-variétés de dimensions supérieures.

Un mot enfin sur les constantes numériques obtenues. Si nous n'avons pas cherché systématiquement à les miminiser, nous nous sommes toutefois efforcés de respecter les ordres de grandeurs naturellement fournis par nos arguments. Ainsi, dans le théorème 1.1, notre méthode nous conduit à une constante du type $\exp \left(c g^{2} \log (g)\right)$, où $c$ est universelle (voir la note de bas de page dans la démonstration du théorème 1.1, à la fin du paragraphe 4.3).

Le théorème 1.3 ci-dessus est un des ingrédients du travail récent de G. RÉMOND (confer [Ré]) sur le dénombrement des points rationnels des sous-variétés de variétés abeliennes. Il est remarquable que dans le cas des courbes, les seuls paramètres intervenant dans la majoration sont, comme pour les théorèmes ci-dessus, le genre de la courbe, le degré d'un corps de définition et la hauteur de la jacobienne de la courbe. Un énoncé conjectural de L. CAPoraso, J. Harris et B. MAzur ( confer $[\mathrm{Ca}-\mathrm{Ha}-\mathrm{Ma}]$ ) prédit que la dépendance en la hauteur devrait même pouvoir être supprimée. Rapportée à notre travail, cette hypothèse semble indiquer que l'intervention du rayon d'injectivité dans la démonstration du théorème 1.1 ne devrait être considérée que comme une défaillance technique de notre méthode et qu'il existe un moyen de contourner la constante de comparaison entre hauteurs projectives et normalisées mentionnées plus haut pour en déduire les théorèmes 1.2 et 1.3 .

Le présent travail est l'exact parallèle abelien de [Da-Phi2], qui lui-même raffine les travaux antérieurs de W. M. SchmidT (confer [Schm]). De même, les travaux de G. RÉmond (confer [Ré]) constituent le pendant abelien des énoncés obtenus dans les tores multiplicatifs et dont on trouvera une présentation dans l'article de synthèse de J.-H. EverTse (confer [Ev]). Citons la version explicite (simplifiée) 
du théorème 2.1 de [Ré] que nous a fourni G. RÉmond en utilisant le théorème 1.4 ci-dessus :

Théorème (G. Rémond). Soient $X \subset A$ une sous-variété algébrique d'une variété abelienne toutes deux définies sur $\overline{\mathbb{Q}}$ et $\Gamma \subset A(\overline{\mathbb{Q}})$ un sous-groupe de rang fini $r$. Alors, avec les notations du théorème 1.4, il existe un entier naturel $S$ satisfaisant

$$
S \leq\left(2^{34} \cdot h_{0}(A) \cdot \operatorname{deg}(X)\right)^{(r+1) g^{5(\operatorname{dim}(X)+1)^{2}}},
$$

des éléments $x_{1}, \ldots, x_{S} \in X(\overline{\mathbb{Q}}) \cap \Gamma$ et des sous-variétés abeliennes $B_{1}, \ldots, B_{S}$ de $A$ tels que $x_{i}+B_{i} \subset X$ pour $i=1, \ldots, S$ et

$$
X(\overline{\mathbb{Q}}) \cap \Gamma=\bigcup_{i=1}^{S}\left(x_{i}+B_{i}\right)(\overline{\mathbb{Q}}) \cap \Gamma .
$$

Le paragraphe 2 introduit les coordonnées de Mumford (confer [Mu]) et explicite les relations qui les lient. Si on les compare aux relations entre fonctions thêta classiques, on vérifie facilement que ce sont les mêmes, à des racines de l'unité près, qui sont sans importance lorsqu'on traite des questions de degré et de hauteur. On utilise les formules obtenues pour majorer l'écart entre hauteurs projective et normalisée d'une sous-variété d'une variété abelienne, de façon totalement explicite. Le paragraphe 3 démontre le théorème 1.1, on y reprend les arguments de [Da-Phi1] mais on fait disparaître beaucoup des paramètres diophantiens en construisant une fonction auxiliaire petite (plutôt que nulle) sur la fibre spéciale et en moyennant les inégalités obtenues sur les translatés de la sous-variété considérée par les points de torsion de la variété abelienne. Le paragraphe 4 est consacré à la descente qui permet de déduire d'une minoration du minimum essentiel une estimation des minimums suivants sur $X^{\circ}$. La démarche, fondée sur une récurrence, est calquée sur celle de [Da-Phi2] qui introduit malheureusement un exposant exponentiel en la dimension de la variété. On en déduit les théorèmes 1.2 et 1.3 ci-dessus. Enfin, l'appendice donne des estimations explicites de croissance des fonctions thêta et minore le rayon d'injectivité en fonction du degré d'un corps de définition et de la hauteur de la variété abelienne sous-jacente.

Nous remercions chaleureusement Gaël RÉMOND pour ses nombreux et pertinents commentaires sur une première version de ce texte, ainsi que l'arbitre de la publication pour son considérable travail exégétique qui nous permet de présenter au lecteur un texte plus compréhensible et notablement épuré.

\section{Notations}

Les questions de hauteurs étant primordiales dans ce texte, nous fixons une fois pour toutes dans ce paragraphe les notions de hauteurs, normes et mesures locales qui prévalent dans l'introduction et dans la suite de notre travail, en indiquant 
les différences (essentiellement de normalisation) avec celles des résultats que nous utiliserons en références.

La notion de hauteur projective des points et des variétés dans les espaces projectifs est celle de [Ph]-III. On la notera $h(x)$ ou $h(X)$ si $x$ et $X$ désignent un point et une variété définis sur $\overline{\mathbb{Q}}$ dans un espace projectif $\mathbb{P}_{N}$. Elle diffère de celle apparaissant dans [Ph]-I par la contribution des places archimédiennes. Précisément, si $\boldsymbol{k}$ est un corps de définition de $X$ et $f_{X}$ une de ses formes éliminantes (ou de CHOw) définie sur $\boldsymbol{k}$, la hauteur projective de $X$ s'écrit en termes de mesures locales

$$
h(X):=\sum_{v} \frac{\left[\boldsymbol{k}_{v}: \mathbb{Q}_{v}\right]}{[\boldsymbol{k}: \mathbb{Q}]} \cdot \log \left(M_{v}\left(f_{X}\right)\right),
$$

où $v$ parcourt l'ensemble des places de $\boldsymbol{k}$, et $\boldsymbol{k}_{v}$ (respectivement $\mathbb{Q}_{v}$ ) désigne le complété de $\boldsymbol{k}$ (respectivement $\mathbb{Q}$ ) pour la place $v$ (respectivement la place induite par $v$ ). La mesure locale $M_{v}$ d'une forme est le maximum des valeurs absolues $v$-adiques des coefficients de la forme pour les places ultramétriques et pour une place archimédienne associée à un plongement $\sigma_{v}$ de $\boldsymbol{k}$ dans $\mathbb{C}$

$$
\log \left(M_{v}\left(f_{X}\right)\right):=\int_{S_{N+1}^{r}} \log \left|\sigma_{v}\left(f_{X}\right)\right| \cdot \sigma_{N+1}^{\wedge r}+d^{\circ} f_{X} \cdot \sum_{i=1}^{N} \frac{1}{2 i},
$$

où $r=\operatorname{dim}(X)+1, S_{N+1}$ désignant pour sa part la sphère unité de $\mathbb{C}^{N+1}$ et $\sigma_{N+1}$ la mesure invariante de masse totale 1 sur $S_{N+1}$. En particulier, la hauteur projective d'un point diffère de la hauteur de WEIL par la contribution des places archimédiennes où la norme euclidienne remplace la norme du maximum d'un système de coordonnées projectives du point. De même, la hauteur projective d'une variété diffère de celle de [Ph]-I par la contribution des places archimédiennes où la mesure $M_{v}$ ci-dessus remplace la classique mesure de MAHLER.

On rappelle que si l'on désigne par $\sigma_{\delta}$ le plongement de VERONESE de l'espace projectif $\mathbb{P}_{N}$ dans l'espace projectif $\mathbb{P}_{N^{\prime}}$ avec $N^{\prime}+1:=\left(\begin{array}{c}N+\delta \\ N\end{array}\right)$ défini par

$$
\begin{aligned}
\sigma_{\delta}: \mathbb{P}_{N} & \longrightarrow \mathbb{P}_{N^{\prime}} \\
\sigma(x) & \longmapsto\left(\cdots:\left(\begin{array}{c}
\delta \\
\alpha
\end{array}\right)^{1 / 2} x^{\alpha}: \cdots\right)_{\substack{\alpha \in \mathbb{N} N+1 \\
|\alpha|=\delta}},
\end{aligned}
$$

on a, avec les notations précédentes, $\operatorname{deg}\left(\sigma_{\delta}(X)\right)=\delta^{r-1} \cdot \operatorname{deg}(X)$ et $h\left(\sigma_{\delta}(X)\right)=$ $\delta^{r} . h(X)$, voir $[\mathrm{Ph}]$-III, page 347 .

Si $\boldsymbol{x}$ est un vecteur à coordonnées dans $\boldsymbol{k}_{v}$ on notera $\|\boldsymbol{x}\|_{v}$ la norme du maximum des coordonnées de $\boldsymbol{x}$ si $v$ est ultramétrique et la norme euclidienne de $\boldsymbol{x}$ si $v$ est archimédienne. Si $\boldsymbol{x}$ désigne un système de coordonnées d'un point projectif $x$, on a

$$
h(x):=\sum_{v} \frac{\left[\boldsymbol{k}_{v}: \mathbb{Q}_{v}\right]}{[\boldsymbol{k}: \mathbb{Q}]} \cdot \log \left(\|\boldsymbol{x}\|_{v}\right)
$$


On utilisera à l'occasion la hauteur de WeIL d'un vecteur $\boldsymbol{x}:=\left(x_{1}, \ldots, x_{M}\right)$

$$
h_{W}(\boldsymbol{x}):=\sum_{v} \frac{\left[\boldsymbol{k}_{v}: \mathbb{Q}_{v}\right]}{[\boldsymbol{k}: \mathbb{Q}]} \cdot \log \max \left(\left|x_{1}\right|_{v}, \ldots,\left|x_{M}\right|_{v}\right),
$$

pour des vecteurs dont les composantes seront les coefficients d'un polynôme, voire d'une famille de polynômes (《hauteur homogène »), mais aussi en adjoignant 1 à ces coefficients (《hauteur inhomogène »).

\section{Plongements et hauteurs normalisées}

Nous allons établir ici des comparaisons effectives entre la hauteur projective d'une sous-variété d'une variété abelienne et la hauteur normalisée correspondante. Dans le cas des points, il s'agit là d'estimations bien connues : en effet, ce problème a été traité par Y. MANin et Y. ZARHin (voir [Ma-Za]), ainsi que par J. TATE et H. Zimmer dans le cas particulier des courbes elliptiques (voir par exemple [Zi]). Dans le cas des variétés de dimensions supérieures, ces questions ont été traitées dans [Ph]-III, §. 3, mais sans expliciter les constantes de comparaison. Nous suivrons les preuves de cette référence, et les compléterons afin d'obtenir des constantes numériques. Ce travail sera effectué au paragraphe 3.3, mais dans un premier temps nous donnons des estimations effectives des degrés et hauteurs des formules de duplication, addition et translations par des points de torsion sur les variétés abeliennes.

Pour plus de généralité, et bien qu'à partir du paragraphe 4 suivant nous nous restreindrons au cas principalement polarisé et n'utiliserons que des estimations archimédiennes (on pourrait donc travailler exclusivement avec des fonctions thêta classiques), nous montrerons ces résultats à l'aide des plongements décrits par Mumford (voir $[\mathrm{Mu}]$ ), en termes de « coordonnées de MumFord », qui ne sont rien d'autre que des avatars algébriques des fonctions thêta. Le paragraphe 3.1 sera donc consacré à une étude préliminaire des coordonnées de MuMFord.

Enfin, comme la technique pour comparer les hauteurs projective et normalisée consiste à étudier la variation de la hauteur sous l'action d'itérés de la multiplication par 2, le paragraphe 3.2 sera consacré à une description explicite des formules de duplication ainsi que de leurs inverses dans les coordonnées de Mumford. Nous donnerons de surcroît une estimation effective de la hauteur des formules d'addition (également obtenues à partir de formules explicites).

\subsection{Coordonnées de Mumford}

Nous commencerons par rappeler le plus succintement possible les notations et définitions de base relatives aux plongements associés à des structures thêta (au paragraphe 3.1.1) ; pour plus de détails, on pourra se reporter à l'article original de D. Mumford (confer [Mu]), ou par exemple au chapitre 6 de [Bi-La]. Nous modi- 
fierons ensuite ces coordonnées projectives, à l'aide d'une transformation linéaire très simple afin d'obtenir des estimations plus agréables, et nous expliciterons le dictionnaire permettant de passer des bases canoniques de MUMFORD à ces nouvelles coordonnées (paragraphe 3.1.2). Au paragraphe suivant 3.1.3, nous précisons les transformations linéaires induites par les translations par certains points de torsion, et enfin, nous conclurons au paragraphe 3.1.4 par une discussion sur le parallélisme avec les fonctions thêta classiques.

\subsubsection{Notations et rappels}

On supposera donnés : une variété abelienne $A$, de dimension $g$, définie sur $\boldsymbol{k}=\overline{\mathbb{Q}}$, un fibré très ample et totalement symétrique $\mathcal{L}$ sur $A$, au sens de la définition $^{2}$ page 305 de $[\mathrm{Mu}$ ( voir aussi [Bi-La], exercice 12, page 180). On notera $\mathcal{H}(\mathcal{L})$ le sous-groupe de $A$ formé des points $x \in A$ tels que $\tau_{x}^{\star} \mathcal{L} \simeq \mathcal{L}$, où $\tau_{x}$ désigne la translation par $x$, et $\mathcal{G}(\mathcal{L})$ l'ensemble des couples $(x, \varphi)$, où $x \in \mathcal{H}(\mathcal{L})$ et $\varphi$ est un isomorphisme $\varphi: \mathcal{L} \stackrel{\sim}{\longrightarrow} \tau_{x}^{\star} \mathcal{L}$. On vérifie alors que l'on a la suite exacte (voir $[\mathrm{Mu}]$, page 290) :

$$
\{1\} \longrightarrow \boldsymbol{k}^{\star} \longrightarrow \mathcal{G}(\mathcal{L}) \longrightarrow \mathcal{H}(\mathcal{L}) \longrightarrow 0,
$$

et que $\boldsymbol{k}^{\star}$ est le centre de $\mathcal{G}(\mathcal{L})$ pour sa structure de groupe naturelle, et l'on peut définir une forme bilinéaire alternée non dégénérée $e^{\mathcal{L}}$ sur $\mathcal{H}(\mathcal{L})$ comme suit :

$$
\left\{\begin{array}{l}
\text { soient } x \text { et } y \text { dans } \mathcal{H}(\mathcal{L}), \text { et } \tilde{x}, \tilde{y} \text { dans } \mathcal{G}(\mathcal{L}) \text { au-dessus de } x, y, \\
\text { on pose }: e^{\mathcal{L}}(x, y)=\tilde{x} \cdot \tilde{y} \cdot \tilde{x}^{-1} \cdot \tilde{y}^{-1} \in \boldsymbol{k}^{\star}
\end{array}\right.
$$

On vérifie également que l'on a une décomposition $\mathcal{H}(\mathcal{L})=\mathcal{K}(\mathcal{L}) \oplus \mathcal{K}^{\prime}(\mathcal{L})$ en sousgroupes isotropes et $e^{\mathcal{L}}$ permet d'identifer $\mathcal{K}^{\prime}(\mathcal{L})$ au dual $\widehat{\mathcal{K}(\mathcal{L})}$ de $\mathcal{K}(\mathcal{L})$ (voir $[\mathrm{Mu}]$, page 293).

La théorie des diviseurs élémentaires appliquée à $\mathcal{K}(\mathcal{L})$ nous donne maintenant un $g$-uplet $\boldsymbol{d}=\left(d_{1}, \ldots, d_{g}\right)$ d'entiers positifs, $d_{i+1} \mid d_{i}, i=1, \ldots, g-1$, et l'on pose

$$
\begin{gathered}
K(\boldsymbol{d})=\bigoplus_{i=1}^{g} \mathbb{Z} / d_{i} \mathbb{Z}, \\
\widehat{K(\boldsymbol{d})}=\operatorname{hom}\left(K(\boldsymbol{d}), \boldsymbol{k}^{\star}\right)
\end{gathered}
$$

et enfin :

$$
H(\boldsymbol{d})=K(\boldsymbol{d}) \oplus \widehat{K(\boldsymbol{d})} .
$$

Le $g$-uplet $\boldsymbol{d}$ sera appelé le type de $\mathcal{L}$.

Avec ces données, on introduit le groupe $G(\boldsymbol{d})$ qui est le produit :

$$
\boldsymbol{k}^{\star} \times K(\boldsymbol{d}) \times \widehat{K(\boldsymbol{d})},
$$

$\overline{2 \text { Définition que nous rappelons ci-dessous. }}$ 
muni d'une loi de groupe via :

$$
(\alpha, x, l) \cdot\left(\alpha^{\prime}, x^{\prime}, l^{\prime}\right)=\left(\alpha \cdot \alpha^{\prime} \cdot l^{\prime}(x), x+x^{\prime}, l . l^{\prime}\right)
$$

et l'on vérifie que l'on a une suite exacte :

$$
\{1\} \longrightarrow \boldsymbol{k}^{\star} \longrightarrow G(\boldsymbol{d}) \longrightarrow H(\boldsymbol{d}) \longrightarrow 0 \text {. }
$$

On définit de même que pour $\mathcal{H}(\mathcal{L})$, par les commutateurs, une forme bilinéaire alternée non dégénérée sur $H(\boldsymbol{d})$ qui admet pour sous-espaces isotropes les $K(\boldsymbol{d})$, $\widehat{K(\boldsymbol{d})}$; on notera cette forme $e^{\boldsymbol{d}}$.

Le groupe $\mathcal{G}(\mathcal{L})$ admet alors une représentation naturelle dans le $\boldsymbol{k}$-espace vectoriel $\Gamma(A, \mathcal{L})$,

$$
\left\{\begin{array}{l}
\text { soit } z=(x, \varphi) \in \mathcal{G}(\mathcal{L}) \text { et } s \in \Gamma(A, \mathcal{L}), \\
\text { on définit } U_{z}: \Gamma(A, \mathcal{L}) \rightarrow \Gamma(A, \mathcal{L}) \text {, en posant : } U_{z}(s)=\tau_{-x}^{\star}(\varphi(s)) .
\end{array}\right.
$$

Cette représentation fait de $\Gamma(A, \mathcal{L})$ un $\mathcal{G}(\mathcal{L})$-module irréductible (confer $[\mathrm{Mu}]$, théorème 2, page 297).

Soit maintenant $V(\boldsymbol{d})$ l'espace des fonctions sur $K(\boldsymbol{d})$ à valeurs dans $\boldsymbol{k}$, on définit une représentation $U$ de $G(\boldsymbol{d})$ dans $V(\boldsymbol{d})$, en posant :

$$
\forall y \in K(\boldsymbol{d}), \quad\left(U_{(\alpha, x, l)}(f)\right)(y)=\alpha \cdot l(y) \cdot f(x+y) .
$$

Cette représentation est également irréductible (ibidem proposition 3, page 295).

On peut alors résumer les résultats de la première partie de l'article de D. MUMFORD $[\mathrm{Mu}]$ en l'énoncé suivant :

Proposition-Définition 3.1. Il existe un isomorphisme de $\mathcal{G}(\mathcal{L})$ vers $G(\boldsymbol{d})$ qui agit trivialement sur le sous-groupe $\boldsymbol{k}^{\star}$. De tels isomorphismes sont en nombre fini. Supposons un tel isomorphisme choisi. Il existe alors un unique (à multiplication par un scalaire non nul près) isomorphisme du $\mathcal{G}(\mathcal{L})$-module $\Gamma(A, \mathcal{L})$ vers le $G(\boldsymbol{d})$ module $V(\boldsymbol{d})$. On appellera un tel choix d'isomorphisme une structure thêta pour la paire $(A, \mathcal{L})$.

Supposons donnée une structure thêta pour $(A, \mathcal{L})$, et soit $f: V(\boldsymbol{d}) \longrightarrow$ $\Gamma(A, \mathcal{L})$ l'isomorphisme induit par cette structure; on identifiera tacitement les données $V(\boldsymbol{d}), G(\boldsymbol{d})$ à $\Gamma(A, \mathcal{L}), \mathcal{G}(\mathcal{L})$ respectivement.

Les fonctions caractéristiques fournissent alors une base naturelle de $V(\boldsymbol{d})$. Soit $a \in K(\boldsymbol{d})$, on définit $\delta_{a}^{\boldsymbol{d}} \in V(\boldsymbol{d})=\Gamma(A, \mathcal{L})$ en posant :

$$
\begin{cases}\delta_{a}^{\boldsymbol{d}}(x)=1 & \text { si } x=a \\ \delta_{a}^{\boldsymbol{d}}(x)=0 & \text { si } x \neq a, x \in K(\boldsymbol{d}) .\end{cases}
$$

La famille de sections globales $\delta^{\mathcal{L}}:=f\left(\delta^{\boldsymbol{d}}\right)=\left(f\left(\delta_{a}^{\boldsymbol{d}}\right)\right)_{a \in K(\boldsymbol{d})}$ nous fournit alors un plongement projectif de $A$ que nous noterons $\tilde{\Theta}_{\mathcal{L}}$. On appelle cette base les coordonnées de Mumford. On notera

$$
\tilde{\Theta}_{\mathcal{L}}(0)=\left(\cdots: q_{\mathcal{L}}(a): \cdots\right)_{a \in K(\boldsymbol{d})}
$$


où $q_{\mathcal{L}}(a)$ est la «valeur en l'origine 0 de $A \gg$ de la section $\delta_{a} \in \Gamma(A, \mathcal{L}) ;$ comme la collection des $q_{\mathcal{L}}(a)$ est définie à un scalaire non nul près, nous disposons bien de coordonnées projectives de l'origine dans le plongement ainsi fixé. Nous supposerons implicitement dans toute la suite que ce scalaire est choisi de telle sorte que les égalités écrites ci-dessous soient correctes ${ }^{3}$.

On note $[-1]$ la multiplication par -1 sur $A$, et l'on suppose donné un isomorphisme $\psi$ entre $\mathcal{L}$ et $[-1]^{\star} \mathcal{L}$ dont la restriction $\psi(0)$ à la fibre de $\mathcal{L}$ au-dessus de l'origine est l'identité. Si $x$ est un point d'ordre 2 de $A$, on note $e_{\star}^{\mathcal{L}}(x)$ le scalaire $\alpha$ tel que la restriction $\psi(x)$ de $\psi$ à la fibre $\mathcal{L}(x)$ de $\mathcal{L}$ au dessus de $x$ soit la multiplication par $\alpha$.

On dit alors qu'un fibré en droites $\mathcal{M}$ sur $A$ est totalement symétrique, s'il est symétrique et si $e_{\star}^{\mathcal{M}}(x)=1$ pour tout point d'ordre 2 de $A$.

Nous allons maintenant rappeler la définition de structures thêta compatibles pour la paire $\left(\mathcal{L}, \mathcal{L}^{\otimes 2}\right)$. Pour ceci, nous aurons besoin des faits suivants.

Reprenons l'isomorphisme $\psi$ fixé précédemment, et considérons pour un point $z=(x, \varphi) \in \mathcal{G}(\mathcal{L})$ la composition :

$$
\mathcal{L} \stackrel{\psi}{\longrightarrow}[-1]^{\star} \mathcal{L} \stackrel{[-1]^{\star} \varphi}{\longrightarrow}[-1]^{\star} \tau_{x}^{\star} \mathcal{L}=\tau_{-x}^{\star}[-1]^{\star} \mathcal{L} \stackrel{\tau_{-x}^{\star} \psi}{\longleftarrow} \tau_{-x}^{\star} \mathcal{L}
$$

on pose alors $\nu_{-1}(z)=\left(-x,\left(\tau_{-x}^{\star} \psi\right)^{-1} \circ\left([-1]^{\star} \varphi\right) \circ \psi\right)$.

On définit ensuite pour tout entier $n \geq 2$, un morphisme $\varepsilon_{n}: \mathcal{G}(\mathcal{L}) \longrightarrow \mathcal{G}\left(\mathcal{L}^{\otimes n}\right)$, en posant

$$
\varepsilon_{n}(x, \varphi)=\left(x, \varphi^{\otimes n}\right)
$$

où $\varphi^{\otimes n}$ est l'isomorphisme

$$
\mathcal{L}^{\otimes n} \stackrel{\varphi^{\otimes n}}{\longrightarrow} \tau_{x}^{\star} \mathcal{L}^{\otimes n}
$$

induit par $\varphi$.

Enfin, nous utiliserons un morphisme $\eta_{n}: \mathcal{G}\left(\mathcal{L}^{\otimes n}\right) \longrightarrow \mathcal{G}(\mathcal{L})$; nous ne rappellerons pas sa définition précise (voir [Mu], page 310), mais dirons simplement qu'il s'agit d'un morphisme canonique rendant commutatif le diagramme :

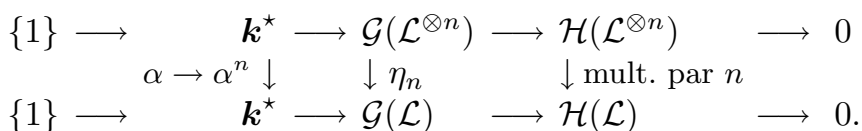

Introduisons maintenant les pendants de ces morphismes sur les groupes $G(\boldsymbol{d})$. Soit $\boldsymbol{d}$ un $g$-uplet de diviseurs élémentaires, on note $2 \boldsymbol{d}$ le $g$-uplet $\left(2 d_{1}, \ldots, 2 d_{g}\right)$, et l'on identifie $K(\boldsymbol{d})$ à un sous-groupe de $K(2 \boldsymbol{d})$ via la flèche

$$
\left(a_{1}, \ldots, a_{g}\right) \longmapsto\left(2 a_{1}, \ldots, 2 a_{g}\right) .
$$

3 Si $A$ est une variété abelienne complexe, plongée dans un espace projectif via un plongement thêta classique, on dispose bien évidemment d'une structure thêta pour le fibré très ample associé au plongement ainsi fixé; nous reviendrons sur ce parallélisme ci-après. 
Le dual $\widehat{K(\boldsymbol{d})}$ est alors naturellement un quotient de $\widehat{K(2 \boldsymbol{d})}$; on notera alors $\bar{l}$ l'image naturelle d'un élément $l$ de $\widehat{K(2 \boldsymbol{d})}$ dans $\widehat{K(\boldsymbol{d})}$; on notera également que si $l \in \widehat{K(\boldsymbol{d})}$, il existe un unique élément $l^{\prime}$ de $\widehat{K(2 \boldsymbol{d})}$ tel que

$$
l^{\prime}(x)=l(2 x)
$$

pour tout élément $x$ de $K(2 \boldsymbol{d})$. On en déduit une injection de $\widehat{K(\boldsymbol{d})}$ dans $\widehat{K(2 \boldsymbol{d})}$ que nous noterons $2 \star$. On note alors $E_{2}: G(\boldsymbol{d}) \longrightarrow G(2 \boldsymbol{d})$ le morphisme défini par

$$
E_{2}((\alpha, x, l))=\left(\alpha^{2}, x, 2 \star l\right) .
$$

Pour $n \in \mathbb{Z}, D_{n}$ est le morphisme de $G(\boldsymbol{d}) \longrightarrow G(\boldsymbol{d})$ donné par

$$
D_{n}((\alpha, x, l))=\left(\alpha^{n^{2}}, n x, l^{n}\right)
$$

et $H_{2}: G(2 \boldsymbol{d}) \longrightarrow G(\boldsymbol{d})$ défini par

$$
H_{2}((\alpha, x, l))=\left(\alpha^{2}, 2 x, \bar{l}\right) .
$$

Avec ces notations, on peut définir : une structure thêta $f: \mathcal{G}(\mathcal{L}) \longrightarrow G(\boldsymbol{d})$ est dite symétrique si

$$
f \circ \nu_{-1}=D_{-1} \circ f
$$

deux structures thêta $f_{1}$ pour $\mathcal{L}$ et $f_{2}$ pour $\mathcal{L}^{\otimes 2}$ sont dites compatibles si elles sont toutes deux symétriques et si

$$
f_{2} \circ \varepsilon_{2}=E_{2} \circ f_{1}, \quad \text { et } \quad f_{1} \circ \eta_{2}=H_{2} \circ f_{2} .
$$

\subsubsection{Petit formulaire}

Nous supposerons pour commencer que le fibré $\mathcal{L}$ est totalement symétrique, et muni d'une paire de structures thêta compatibles pour $\left(\mathcal{L}, \mathcal{L}^{\otimes 2}\right)$. Notons $\xi$ l'isogénie :

$$
\begin{aligned}
\xi: A \times A & \longrightarrow A \times A \\
(x, y) & \longmapsto(x+y, x-y) .
\end{aligned}
$$

Notons $\mathcal{N}$ le fibré $\pi_{1}^{\star} \mathcal{L} \otimes \pi_{2}^{\star} \mathcal{L}$; l'isogénie $\xi$ induit alors le morphisme :

$$
\begin{aligned}
\Gamma(A, \mathcal{L}) \otimes \Gamma(A, \mathcal{L}) \stackrel{\mathrm{KüNNETH}}{\simeq} & \Gamma\left(A^{2}, \mathcal{N}\right) \\
& \downarrow \xi^{\star} \\
& \Gamma\left(A^{2}, \xi^{\star} \mathcal{N}\right) \simeq \Gamma\left(A^{2}, \mathcal{N}^{\otimes 2}\right) \stackrel{\text { KÜNNETH }}{\simeq} \Gamma\left(A, \mathcal{L}^{\otimes 2}\right) \otimes \Gamma\left(A, \mathcal{L}^{\otimes 2}\right) .
\end{aligned}
$$

Les structures thêta induisent un morphisme $V(\boldsymbol{d})^{2} \longrightarrow V(2 \boldsymbol{d})^{2}$, dont la description en termes de bases canoniques permet de décrire entièrement l'action de $\xi^{\star}$. Plus précisément, la «formule d'addition fondamentale » (confer [Mu], page 324) une fois traduite (confer également [Bi-La], exercice 1, page 208 pour la traduction) donne l'égalité suivante pour tout $(a, b) \in K(\boldsymbol{d})^{2}$ :

$$
\delta_{a}^{\mathcal{L}}(x+y) \delta_{b}^{\mathcal{L}}(x-y)=\sum_{\eta \in K(2)} \delta_{\frac{a+b}{2}+\eta}^{\mathcal{L}^{\otimes 2}}(x) \delta_{\frac{a-b}{2}+\eta}^{\mathcal{L}^{\otimes 2}}(y),
$$


où, pour plus de lisibilité, nous avons désigné par $x$, respectivement par $y$, la «première » (respectivement la «seconde ») coordonnée dans $A^{2}$. Enfin, dans la relation ci-dessus, $K(2)$ désigne l'ensemble des points de 2-torsion de $K(\boldsymbol{d})$ (rappelons que puisque $\mathcal{L}$ est totalement symétrique, $K(2) \simeq(\mathbb{Z} / 2 \mathbb{Z})^{g}$ en vertu du corollaire 4 , page 315 de $[\mathrm{Mu}]$ ), et $\frac{a+b}{2}$ (respectivement $\frac{a-b}{2}$ ) désigne un point de 2-division quelconque de $a+b$ (respectivement $a-b$ ), sous la seule restriction $\frac{a+b}{2}+\frac{a-b}{2}=a$.

La multiplication naturelle

$$
\Gamma(A, \mathcal{L}) \otimes \Gamma(A, \mathcal{L}) \longrightarrow \Gamma\left(A, \mathcal{L}^{\otimes 2}\right)
$$

est également convertie en une loi de composition :

$$
\text { - : } V(\boldsymbol{d}) \otimes V(\boldsymbol{d}) \stackrel{\bullet}{\longrightarrow} V(2 \boldsymbol{d}) \text {. }
$$

Nous utiliserons les relations suivantes (confer [Ma-Za], page 172, formules (1) et $(2))$

$$
\delta_{a}^{\mathcal{L}} \bullet \delta_{b}^{\mathcal{L}}=\sum_{\eta \in K(2)} q_{\mathcal{L} \otimes 2}\left(\frac{a-b}{2}+\eta\right) \delta_{\frac{a+b}{2}+\eta}^{\mathcal{L}^{\otimes 2}},
$$

où, là encore, les éléments $\frac{a-b}{2}, \frac{a+b}{2}$ sont choisis de telle sorte que leur somme vaut $a$, tous les autres choix étant arbitraires.

Enfin, si l'on suppose que l'on a de plus une structure thêta sur $\mathcal{L}^{\otimes 4}$ donnant une paire de structures compatibles pour $\left(\mathcal{L}^{\otimes 2}, \mathcal{L}^{\otimes 4}\right)$, on dispose de la « formule de duplication »:

$$
\delta_{a}^{\mathcal{L}} \circ[2]=\sum_{\eta \in K(2)} \delta_{\frac{a}{2}+\eta}^{\mathcal{L}}{ }^{\otimes 4}
$$

On a également

$$
q_{\mathcal{L}}(b) \delta_{a}^{\mathcal{L}} \circ[2]=\sum_{\eta \in K(2)} \delta_{\frac{a+b}{2}+\eta}^{\mathcal{L}^{\otimes 2}} \bullet \delta_{\frac{a-b}{2}+\eta}^{\mathcal{L}^{\otimes 2}} .
$$

On déduit facilement ces relations de la formule d'addition fondamentale (formule (3)) rappelée ci-dessus.

Nous allons maintenant introduire des coordonnées modifiées. Ainsi, pour toute la suite, on fixera un fibré ample et symétrique $\mathcal{M}$ sur $A$, et l'on posera ${ }^{4} \mathcal{L}=$ $\mathcal{M}^{\otimes 4}$, de telle sorte que $\mathcal{L}$ est automatiquement totalement symétrique (voir $[\mathrm{Mu}]$, page 307). On suppose de plus données des paires de structures thêta compatibles pour $\left(\mathcal{L}, \mathcal{L}^{\otimes 2}\right),\left(\mathcal{L}^{\otimes 2}, \mathcal{L}^{\otimes 4}\right),\left(\mathcal{L}^{\otimes 4}, \mathcal{L}^{\otimes 8}\right)$ et $\left(\mathcal{L}^{\otimes 8}, \mathcal{L}^{\otimes 16}\right)$ (on renvoie à $[\mathrm{Mu}]$ pages 317

\footnotetext{
$\overline{4}$ On pourrait bien sûr affaiblir cette contrainte et prendre pour $\mathcal{L}$ un fibré très ample totalement symétrique quelconque, mais cette restriction ne fait pas véritablement perdre en généralité pour la suite de ce travail puisque la variation des hauteurs projectives est bien contrôlée sous l'action d'un Veronese, et simplifie de surcroît l'expression des formules de duplication en assurant l'existence de suffisamment de points de $2^{n}$-torsion dans le groupe $\mathcal{H}(\mathcal{L})$.
} 
à 320 pour l'existence de telles structures, et en particulier à la proposition 7 , page 320$)$.

On notera $K_{i}=K\left(2^{i} \boldsymbol{d}\right)$ pour $0 \leq i \leq 4$, nous identifierons $\Gamma\left(A, \mathcal{L}^{\otimes 2^{i}}\right)$ à $V\left(2^{i} \boldsymbol{d}\right)$ et nous fixerons des coordonnées de MumFord, que nous noterons $\delta^{(i)}=$ $\left(\delta_{a}^{(i)}\right)_{a \in K_{i}}=\left(\delta_{a}^{\left(2^{i} d\right)}\right)_{a \in K_{i}}$, pour $\Gamma\left(A, \mathcal{L}^{\otimes 2^{i}}\right)$.

Nous noterons également pour $n$ entier $K_{i}(n)(i=0, \ldots, 4)$ le sous-groupe de $K_{i}$ formé des éléments de $n$-torsion. On notera que le choix $\mathcal{L}=\mathcal{M}^{\otimes 4}$, où $\mathcal{M}$ est ample et symétrique impose que le groupe $\mathcal{H}(\mathcal{L})$ contient l'ensemble $A_{4}$ des points de 4 -torsion de $A$ (confer par exemple [Bi-La], chapitre 2, lemme 4.7, page 38). En particulier, pour tout $n$ divisant 4 on a $K_{0}(n)=\cdots=K_{4}(n) \simeq(\mathbb{Z} / n \mathbb{Z})^{g}$ et nous noterons parfois $K(n)$ ce sous-groupe.

On introduit maintenant les coordonnées modifiées suivantes : soient $(a, l) \in$ $K_{0} \times \widehat{K(2)}$. On pose ${ }^{5}$

$$
\Delta_{(a, l)}^{(0)}=\sum_{c \in K(2)} l(c) \delta_{a+c}^{(0)}
$$

et de même, pour $(a, l) \in K_{2} \times \widehat{K_{2}(4)}$ ou $(a, l) \in K_{4} \times \widehat{K_{4}(8)}$,

$$
\left\{\begin{array}{l}
\Delta_{(a, l)}^{(2)}=\sum_{c \in K_{2}(4)} l(c) \delta_{a+c}^{(2)}, \\
\Delta_{(a, l)}^{(4)}=\sum_{c \in K_{4}(8)} l(c) \delta_{a+c}^{(4)} .
\end{array}\right.
$$

Pour $i=0,2$ ou 4 , on désignera par $\mathcal{Z}_{i}$ un système de représentants du quotient $K_{i} / K_{i}\left(2 \cdot 2^{i / 2}\right)$. Les systèmes de coordonnées que nous choisissons ${ }^{6}$ sont alors :

$$
\Delta^{(i)}=\left(\Delta_{(a, l)}^{(i)}\right)_{\left.a \in \mathcal{Z}_{i}, l \in K_{i} \widehat{\left(2 \cdot 2^{i} / 2\right.}\right)} .
$$

Un tel système de coordonnées définit un plongement projectif que nous notons $\Theta_{\mathcal{L}^{\otimes 2^{i}}}$ (toujours pour $i=0,2$ ou 4 ). On posera également pour tout $(a, l) \in$ $\left.K_{i} \times K_{i} \widehat{\left(2 \cdot 2^{i} / 2\right.}\right)($ alias des très classiques «thêta nullwerte »)

$$
\theta_{\mathcal{L}^{\otimes 2^{i}}}(a, l)=\sum_{c \in K_{i}\left(2 \cdot 2^{i / 2}\right)} l(c) q_{\mathcal{L}^{\otimes 2^{i}}}(a+c),
$$

un système de coordonnées projectives de l'origine dans ce plongement est alors :

$$
\Theta_{\mathcal{L}^{\otimes 2^{i}}}(0)=\left(\cdots: \theta_{\mathcal{L}^{\otimes 2^{i}}}(a, l): \cdots\right)_{a \in \mathcal{Z}_{i}, l \in K_{i}} \widehat{\left(2 \cdot 2^{i / 2}\right)} .
$$

\footnotetext{
5 On effectue donc une transformée de Fourier partielle; d'autres choix sont possibles (confer $[\mathrm{Mu}]$, page 334).

${ }_{6}^{6}$ Qui dépendent donc d'un choix de $\mathcal{Z}_{i}$, mais uniquement à des multiplications par des racines de l'unité près : voir le fait 3.3 , plus bas.
} 
Notation 3.2. On désignera par $h(A)$ la hauteur projective du point $\Theta_{\mathcal{L}^{\otimes 4}}\left(0_{A}\right)=$ $\Theta_{\mathcal{M} \otimes 16}\left(0_{A}\right)$. Pour alléger la notation on désignera parfois indifféremment par $0_{A}$ l'origine de $A$, son image par le plongement projectif $\Theta_{\mathcal{L}{ }^{\otimes 4}}$ et le système de coordonnées projectives $\left(\cdots: \theta_{\mathcal{L}^{\otimes 4}}(a, l): \cdots\right)_{a \in \mathcal{Z}_{2}, l \in \widehat{K_{2}(4)}}$ de ce dernier point.

Terminons ce sous-paragraphe en rappelant les propriétés de base des coordonnées de Mumford une fois traduites dans les nouvelles coordonnées $\Delta$ que nous venons d'introduire ci-dessus.

Fait 3.3. Pour tous quintuplets de structures thêta deux à deux compatibles comme ci-dessus, on a les propriétés suivantes :

(i) Pour $i=0,2$ ou 4 et pour tout $a \in K_{i}$ on a les formules d'inversion ${ }^{7}$ :

$$
\delta_{a}^{(i)}=\frac{1}{2^{(1+i / 2) g}} \sum_{l \in K_{i}\left(2 \cdot 2^{i} / 2\right)} \Delta_{a, l}^{(i)} ;
$$

(ii) pour $i=0,2$, et pour tout $(a, l) \in K_{i} \times K_{i} \widehat{\left(2 \cdot 2^{i} / 2\right)}$, les formules de duplication sont :

$$
\Delta_{a, l}^{(i)} \circ[2]=\Delta_{\left(\frac{a}{2}, 2 \star l\right)}^{(i+2)},
$$

où $\frac{a}{2}$ est un point de 2-division quelconque de a (comparer avec le point (iv) ci-dessous). Rappelons que $2 \star$ est l'injection de $K_{i} \widehat{\left(2 \cdot 2^{i} / 2\right)}$ vers $\left.K_{i} \widehat{\left(4 \cdot 2^{i} / 2\right.}\right)$ définie au paragraphe 3.1.1;

(iii) pour $i=0,2$ ou 4 et pour tout $\left.(a, l) \in K_{i} \times K_{i} \widehat{\left(2 \cdot 2^{i}\right.} / 2\right)$, l'action de la multiplication par -1 est donnée par :

$$
\Delta_{-a, l^{-1}}^{(i)} \circ[-1]=\Delta_{a, l}^{(i)}
$$

(iv) soit $a \in K_{i}($ pour $i=0,2$ ou 4$), u \in K_{i}\left(2 \cdot 2^{i / 2}\right)$ et $\left.l \in K_{i} \widehat{\left(2 \cdot 2^{i} / 2\right.}\right)$; on a alors (variation du système $\left(\Delta_{(a, l)}^{(i)}\right)$ avec $\left.\mathcal{Z}_{i}\right)$ :

$$
\Delta_{(a+u, l)}^{(i)}=l(-u) \Delta_{(a, l)}^{(i)} .
$$

Démonstration. Calculons

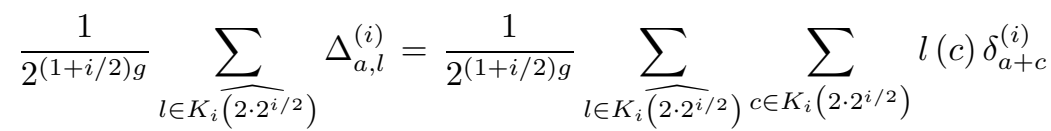

$$
\begin{aligned}
& =\frac{1}{2^{(1+i / 2) g}} \sum_{c \in K_{i}\left(2 \cdot 2^{i / 2}\right)} \delta_{a+c}^{(i)} \sum_{l \in K_{i}\left(2 \cdot 2^{i} / 2\right)} l(c) \text {; }
\end{aligned}
$$

\footnotetext{
7 On notera qu'il convient de corriger comme ci-dessous les formules au bas de la page 176 de [Ma-Za], ainsi que les calculs subséquents de cette référence.
} 
la formule des caractères donne maintenant : $\sum_{l \in K_{i} \widehat{\left(2 \cdot 2^{i} / 2\right)}} l(c)=0$ si $c \neq 0$ et $2^{(1+i / 2) g}$ sinon, ce qui montre (i).

Le point (iii) découle de la formule d'inversion de Mumford, d'où l'on déduit :

$$
\delta_{a} \circ[-1]=\delta_{-a}
$$

(voir [Mu], page 331, voir aussi [Bi-La], exercice 13, page 181); il suffit ensuite de reporter cette relation dans les définitions (7) et (8) des coordonnées $\Delta$.

Passons maintenant au point (ii). On a par définition :

$$
\Delta_{a, l}^{(i)} \circ[2]=\sum_{c \in K_{i}\left(2 \cdot 2^{i / 2}\right)} l(c) \delta_{a+c}^{(i)} \circ[2],
$$

et, par la formule (5),

$$
\Delta_{a, l}^{(i)} \circ[2]=\sum_{c \in K_{i}\left(2 \cdot 2^{i / 2}\right)} l(c) \sum_{\eta \in K(2)} \delta_{\frac{a}{2}+\frac{c}{2}+\eta}^{(2+i)}=\sum_{c \in K_{i+2}\left(4 \cdot 2^{i / 2}\right)} l(2 c) \delta_{\frac{a}{2}+c}^{(2+i)},
$$

et par définition même de l'injection $2 \star$, on a $l(2 c)=2 \star l(c)$, d'où

$$
\Delta_{a, l}^{(i)} \circ[2]=\sum_{c \in K_{i+2}\left(4 \cdot 2^{i / 2}\right)} 2 \star l(c) \delta_{\frac{a}{2}+c}^{(2+i)}=\Delta_{\left(\frac{a}{2}, 2 \star l\right)}^{(2+i)},
$$

d'où le point (ii).

Passons maintenant au point (iv). On a par définition,

$$
\Delta_{(a+u, l)}^{(i)}=\sum_{c \in K_{i}\left(2 \cdot 2^{i / 2}\right)} l(c) \delta_{a+u+c}^{(i)}=\sum_{c \in K_{i}\left(2 \cdot 2^{i / 2}\right)} l(c-u) \delta_{a+c}^{(i)}=l(-u) \Delta_{(a, l)}^{(i)},
$$

d'où le point (iv), et le fait 3.3.

\subsubsection{Action du groupe $\mathcal{H}(\mathcal{L})$ sur les coordonnées de Mumford}

Le groupe thêta $\mathcal{G}(\mathcal{L})$ et sa représentation $U$ permettent d'étudier les propriétés du plongement $\Theta_{\mathcal{L}}$. Cette remarque nous sera utile au paragraphe 3.3 suivant pour étudier la variation de la hauteur projective d'une sous-variété de $A$ définie sur $\overline{\mathbb{Q}}$ par translation par des points de deux torsion (voir lemme 3.10). En effet, si $z=(x, \varphi) \in \mathcal{G}(\mathcal{L})$, on a un automorphisme $U_{z}$ de $\Gamma(A, \mathcal{L})$ et l'on vérifie à l'aide de la définition de $U$ que l'automorphisme projectif associé ne dépend que de $x \in \mathcal{H}(\mathcal{L})$, ce qui nous définit une représentation

$$
\rho_{0} \quad: \mathcal{H}(\mathcal{L}) \longrightarrow \mathrm{PGl}_{N}(\boldsymbol{k}) .
$$

On a alors (voir [Bi-La], page 163, proposition (6.1)) : pour tout $x \in \mathcal{H}(\mathcal{L})$, le diagramme suivant commute :

$$
\begin{aligned}
& A \stackrel{\Theta_{\mathcal{L}}}{\longrightarrow} \\
& \mathbb{P}_{N} \\
& \tau_{-x} \downarrow \downarrow \rho_{0}(x) \\
& A \stackrel{\Theta_{\mathcal{L}}}{\longrightarrow} \mathbb{P}_{N} .
\end{aligned}
$$


On peut bien évidemment en faire autant pour les représentations $U_{i}, i=2$ ou 4 , associées à $\mathcal{G}\left(\mathcal{L}^{\otimes 2^{i}}\right)$; on notera $\rho_{i}$ la représentation associée à chacun de ces plongements.

On en déduit la proposition :

Proposition 3.4. Soient $x$ un élément de $\mathcal{H}\left(\mathcal{L}^{\otimes 2^{i}}\right)$ et $i=0,2$ ou 4 alors, pour tout $a \in \mathcal{Z}_{i}$ et tout $\left.k \in K_{i} \widehat{\left(2 \cdot 2^{i} / 2\right.}\right)$, on $a$ :

$$
\rho_{i}(x)\left[\Delta_{(a, k)}^{(i)}\right]=l(a-u) \Delta_{(a-u, \pi(l) \cdot k)}^{(i)},
$$

où $x$ est identifié à un élément $(u, l)$ de $H\left(2^{i} \boldsymbol{d}\right)=K_{i} \times \widehat{K_{i}}$ et où $\pi$ est la projection $\left.\widehat{K_{i}} \longrightarrow \widehat{K_{i}} /\left(2^{1+\frac{i}{2}} \widehat{K_{i}}\right) \simeq K_{i} \widehat{\left(2 \cdot 2^{i / 2}\right.}\right)$.

En particulier, l'action de $\rho_{i}(x)$ sur le système de coordonnées $\Delta^{(i)}$ est une transformation linéaire donnée par la composée d'une action diagonale agissant par multiplication par des racines de l'unité avec une permutation des coordonnées.

Démonstration. On vérifie (voir par exemple [Bi-La], pages 168-169) que pour tout $a \in K_{i}$ et tout $y \in K_{i}$, on a

$$
\rho_{i}(u, l) \delta_{a}^{(i)}(y)=l(y) \delta_{a}^{(i)}(u+y),
$$

ce qui se traduit par :

$$
\rho_{i}(u, l) \delta_{a}^{(i)}=l(a-u) \delta_{a-u}^{(i)},
$$

en reportant dans la définition de $\Delta^{(i)}$, on en déduit que pour tout $a \in K_{i}$ et tout $\left.k \in K_{i} \widehat{\left(2 \cdot 2^{i} / 2\right.}\right)$, on a :

$$
\begin{aligned}
\rho_{i}(u, l) \Delta_{a, k}^{(i)} & =\sum_{c \in K_{i}\left(2 \cdot 2^{i / 2}\right)} k(c) l(a+c-u) \delta_{a+c-u}^{(i)} \\
& =l(a-u) \Delta_{a-u, \pi(l) \cdot k}^{(i)},
\end{aligned}
$$

ce qui montre bien la proposition 3.4.

Afin de pouvoir tirer parti du point (ii) de la proposition 3.8 ci-après, pour obtenir des informations sur la hauteur des formules de duplication, nous aurons besoin du lemme :

Lemme 3.5. Soit $(a, l) \in K_{2} \times \widehat{K_{2}(4)}$, il existe alors un élément $(b, k) \in K_{2} \times$ $\widehat{K_{2}(4)}$ tel que $b=a \bmod \left(2 K_{2}\right), k^{-1} \cdot l \in{\widehat{K_{2}(4)}}^{2}$ et :

$$
\theta_{\mathcal{L}^{\otimes 4}}(b, k) \neq 0 .
$$

Démonstration. Soit $x \in \mathcal{H}\left(\mathcal{L}^{\otimes 4}\right)$ tel que $x=\left(-a, l^{\prime}\right)$ avec $\pi\left(l^{\prime}\right)=l$, où $\pi$ est la projection de $\widehat{K_{2}}$ sur $\widehat{K_{2}(4)}$, puisque $\mathcal{L}$ est très ample (rappelons que c'est la 
puissance quatrième d'un fibré ample), il existe un élément $(u, \alpha) \in K_{0} \times \widehat{K_{0}(2)}$ tel que :

$$
\Delta_{(u, \alpha)}^{(0)}(-2 x) \neq 0
$$

le fait 3.3, point (ii), nous assure que

$$
\Delta_{\left(\frac{u}{2}, 2 \star \alpha\right)}^{(2)}=\Delta_{(u, \alpha)}^{(0)} \circ[2] .
$$

La proposition 3.4 ci-dessus, appliquée avec $i=2,(a, k)$ remplacé par $\left(\frac{u}{2}, 2 \star \alpha\right)$ et $(u, l)$ remplacé par $\left(-a, l^{\prime}\right)$, nous assure que :

$$
\rho_{2}(x) \Delta_{\left(\frac{u}{2}, 2 \star \alpha\right)}^{(2)}=l^{\prime}\left(\frac{u}{2}+a\right) \Delta_{\left(\frac{u}{2}+a, l \cdot 2 \star \alpha\right)}^{(2)},
$$

et l'on déduit des deux relations précédentes que

$$
\begin{aligned}
\theta_{\mathcal{L} \otimes 4}\left(\frac{u}{2}+a, l \cdot 2 \star \alpha\right) & =l^{\prime}\left(-a-\frac{u}{2}\right) \Delta_{\left(\frac{u}{2}, 2 \star \alpha\right)}^{(2)}(-x) \\
& =l^{\prime}\left(-a-\frac{u}{2}\right) \Delta_{(u, \alpha)}^{(0)}(-2 x) \neq 0 .
\end{aligned}
$$

Par ailleurs, on a bien $2 \star \alpha \in{\widehat{K_{2}(4)}}^{2}$ et $\frac{u}{2}$ est un élément de $K_{1}$ donc de $2 K_{2}$, ce qui montre bien le lemme 3.5 .

\subsubsection{Comparaison avec les thêta classiques}

Supposons que la variété abelienne $A$ est définie sur $\mathbb{C}$. Supposons également pour simplifier la présentation que $\mathcal{M}$ est un fibré symétrique et ample associé à une polarisation principale (pour une discussion dans un cadre plus général, on pourra par exemple se reporter à $[\mathrm{Ig}])$. On peut alors identifier $A$ à un tore complexe $\mathbb{C}^{g} / \Lambda$ où $\Lambda=\mathbb{Z}^{g}+\tau \mathbb{Z}^{g}$, et $\tau$ est dans l'espace de SIEGEL de dimension $g$ (voir appendice). Soit $n$ un entier $\geq 3$ (de telle sorte que $\mathcal{M}^{\otimes n}$ est très ample), on peut alors plonger $A$ dans un espace projectif via l'application

$$
\begin{aligned}
\tilde{\Theta}_{\mathcal{M} \otimes n}: \mathbb{C}^{g} & \longrightarrow \mathbb{P}_{n^{g}-1} \\
z & \longmapsto\left(\theta_{(a, 0)}(n \tau, n z)\right)_{a \in \frac{1}{n} \mathbb{Z}^{g}},
\end{aligned}
$$

où, si $a, b$ sont des éléments de $\mathbb{R}^{g}$, la fonction $\theta_{(a, b)}$ est définie par :

$$
\theta_{(a, b)}(\tau, z):=\sum_{m \in \mathbb{Z}^{g}} \exp \left(\mathrm{i} \pi^{t}(m+a) \tau(m+a)+2 \mathrm{i} \pi^{t}(m+a) \cdot(z+b)\right) .
$$

Si l'on suppose de surcroît que $n$ est pair, alors le fibré $\mathcal{M}^{\otimes n}$ est totalement symétrique et, si l'on note $\boldsymbol{n}$ le $g$-uplet $(n, \ldots, n)$, on peut vérifier facilement que l'application $V(\boldsymbol{n}) \longrightarrow \Gamma\left(A, \mathcal{M}^{\otimes n}\right)$ définie par $\delta_{a} \longmapsto \theta_{(a, 0)}(n \tau, n z)$ pour tout $a \in K(\boldsymbol{n})$ est une structure thêta sur $\mathcal{M}^{\otimes n}$ au sens de la proposition-définition 3.1. De plus, ce même énoncé nous assure qu'une telle identification est unique à une ambiguïté finie près. 
Les plongements $\tilde{\Theta}_{\mathcal{M} \otimes n}$ fournissent aussi naturellement des paires de structures thêta compatibles pour $\left(\mathcal{M}^{\otimes n}, \mathcal{M}^{\otimes 2 n}\right)$. De ce fait toutes les propriétés et formules des paragraphes précédents ont un avatar classique (en particulier la formule d'addition fondamentale (3) correspond au théorème 2, page 139 de [Ig], bien que pour la retrouver à partir de loc. cit., il faille une petite contorsion).

Ainsi, les coordonnées $\Delta$ ne sont qu'un avatar des plongements thêta « avec caractéristique ». Nous supposerons (cela est suffisant pour nos besoins) dorénavant que $n$ est de la forme $n=4^{k}$. On introduit alors le plongement $\Theta_{\mathcal{M} \otimes n}$ qui est défini par la collection des $\left(\theta_{(a, b)}\left(\tau, 2^{k} z\right)\right)_{a, b \in \frac{1}{2^{k}} \mathbb{Z}^{g} / \mathbb{Z}^{g}}$.

Comme les coordonnées $\Delta$, ces dernières sont obtenues à partir $\operatorname{des} \theta_{(a, 0)}(n \tau, n z)$ à l'aide d'une transformée de FoURIER partielle (confer [Ig], page 171) :

$$
\begin{aligned}
\theta_{(a, b)}\left(\tau, 2^{k} z\right) & =\sum_{q \in \mathbb{Z}^{g} / 2^{k} \mathbb{Z}^{g}} \exp \left(2 \mathrm{i} \pi^{t}(q+a) \cdot b\right) \theta_{\left(\frac{q+a}{2^{k}}, 0\right)}\left(4^{k} \tau, 4^{k} z\right) \\
& =\exp \left(2 \mathrm{i} \pi^{t} a \cdot b\right) \cdot \Delta_{\left(\frac{a}{2^{k}}, 2^{k} b\right)}^{(2(k-1))}(z)
\end{aligned}
$$

où $a / 2^{k}$ est un point de $2^{k}$-division quelconque de $a$.

Comme on le voit ci-dessus, les coordonnées $\Delta$ ne coïncident pas vraiment avec les coordonnées induites par les fonctions thêta classiques. En particulier, $\theta_{(a, b)}(\tau,$.$) dépend du choix de b$ dans sa classe modulo $\mathbb{Z}^{g}$ par une multiplication par une racine de l'unité, alors que pour $\Delta_{(a, l)}$, c'est une fonction de $l$ vu dans le quotient $\widehat{K_{0}} / 4 \widehat{K_{0}} \simeq \widehat{K(4)}$. Il s'agit toutefois grosso modo de la même notion, à ces petites ambiguïtés près. On verra d'ailleurs ci-dessous que les formules de RiEmann satisfaites par les coordonnées $\Delta$ sont exactement les mêmes que celles satisfaites par les fonctions thêta classiques.

On peut retrouver les fonctions thêta classiques de manière algébrique en modifiant légèrement la définition des $\Delta$; il suffit de poser pour $x \in \mathcal{H}\left(\mathcal{M}^{\otimes n}\right)$ identifié à un élément $(a, l)$ de $K(\boldsymbol{n}) \times \widehat{K(\boldsymbol{n})}$

$$
\tilde{\Delta}_{(-a, l)}^{(2(k-1))}=\rho_{k-1}(x)\left[\Delta_{(0,0)}^{(2(k-1))}\right] .
$$

En tout état de cause, on pourra par la suite et pour chaque place archimédienne $v$ du corps de nombres sur laquelle $A$ sera supposée définie, identifier $A\left(\mathbb{C}_{v}\right)$ à la variété abelienne complexe plongée via $\Theta_{\mathcal{M} \otimes n}$. On disposera alors de toutes les estimations de comparaison de hauteur que nous allons établir au paragraphe 3.3, puisque les $\Theta_{\mathcal{M} \otimes n}$ satisfont toutes les hypothèses requises (tours de structures thêta compatibles) pour établir ces comparaisons.

\subsection{Formules d'addition et de duplication}

Pour déduire des formules explicites pour les formules d'addition et de duplication, il est agréable de passer aux coordonnées de MumFord modifiées, que nous 
avons introduites. Il est en fait préférable d'établir préalablement pour ces nouvelles coordonnées un analogue des «formules de RIEMANN » classiques (confer par exemple [Ig], theorem 1, page 137), dont nous pourrons déduire, par spécialisation à des cas particuliers les formules recherchées. Pour ceci, nous allons introduire le morphisme :

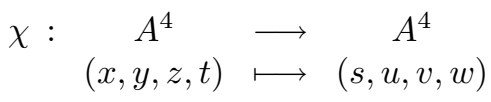

où $s:=x+y+z+t, u:=x+y-z-t, v:=x-y+z-t$ et $w:=x-y-z+t$.

Avec ces notations, on a :

Proposition 3.6. Soient $a_{1}, \ldots, a_{4}$ des éléments de $K_{2}$ et $l_{1}, \ldots, l_{4}$ des éléments de $\widehat{K_{2}(4)}$. Notons $b_{1}=a_{1}+a_{2}+a_{3}+a_{4}, b_{2}=a_{1}+a_{2}-a_{3}-a_{4}, b_{3}=a_{1}-a_{2}+a_{3}-a_{4}$, $b_{4}=a_{1}-a_{2}-a_{3}+a_{4}$; on choisira également un relevé quelconque $\tilde{l}_{i}$ de $l_{i}$ dans $\widehat{K_{4}(8)}$ pour chaque $1 \leq i \leq 4$. Enfin, on posera $\tilde{k}_{1}=\tilde{l}_{1} \cdot \tilde{l}_{2} \cdot \tilde{l}_{3} \cdot \tilde{l}_{4}, \tilde{k}_{2}=\tilde{l}_{1} \cdot \tilde{l}_{2} \cdot \tilde{l}_{3}^{-1} \cdot \tilde{l}_{4}^{-1}$, $\tilde{k}_{3}=\tilde{l}_{1} \cdot \tilde{l}_{2}^{-1} \cdot \tilde{l}_{3} \cdot \tilde{l}_{4}^{-1}$ et $\tilde{k}_{4}=\tilde{l}_{1} \cdot \tilde{l}_{2}^{-1} \cdot \tilde{l}_{3}^{-1} \cdot \tilde{l}_{4}$. Alors, on a la relation :

$$
\begin{aligned}
& 2^{g} \Delta_{\left(a_{1}, l_{1}\right)}^{(2)}(s) \Delta_{\left(a_{2}, l_{2}\right)}^{(2)}(u) \Delta_{\left(a_{3}, l_{3}\right)}^{(2)}(v) \Delta_{\left(a_{4}, l_{4}\right)}^{(2)}(w)= \\
& \sum_{\substack{d \in K(4) / K(2) \\
u \in \widehat{K_{2}(2)}}} l_{1}(d) \Delta_{\left(\frac{b_{1}+d}{4}, \tilde{k}_{1} \cdot 4 \star u\right)}^{(4)}(x) \Delta_{\left(\frac{b_{2}+d}{4}, \tilde{k}_{2} \cdot 4 \star u\right)}^{(4)}(y) \Delta_{\left(\frac{b_{3}+d}{4}, \tilde{k}_{3} \cdot 4 \star u\right)}^{(4)}(z) \Delta_{\left(\frac{b_{4}+d}{4}, \tilde{k}_{4} \cdot 4 \star u\right)}^{(4)}(t),
\end{aligned}
$$

où $\frac{b_{i}+d}{4}$ pour $i=1,2,3,4$ sont choisis de sorte que leur somme vaille $a_{1}$, tous les autres choix étant arbitraires.

Remarques. On notera que dans la formule de RiEmAnn classique, on ne change pas de fibré ; on pourrait penser à utiliser le fait 3.3, point (ii), mais rien ne permet d'assurer a priori que les $b_{i} / 2$ sont des éléments de $K_{2}$, d'où la formulation que nous avons choisie.

Plus généralement, la formule de RIEMANN classique autorise des « coordonnées thêta avec caractéristiques réelles quelconques » alors qu'ici nous ne disposons bien évidemment que de coordonnées dans $K_{2} \times \widehat{K_{2}(4)}$. Pour obtenir une telle variante, il suffit toutefois, en notant $\mathcal{P}$ le fibré de PoInCARÉ sur $A \times \widehat{A}$, de remplacer le fibré $\mathcal{N}=\otimes_{i=1}^{4} \pi_{i}^{\star} \mathcal{L}$ par un fibré de la forme $\mathcal{N}^{\prime}:=\otimes_{i=1}^{4} \pi_{i}^{\star} \mathcal{L}_{i}$ où pour $i=1, \ldots, 4$ on choisit $\mathcal{L}_{i}=\mathcal{L} \otimes \mathcal{P}_{\mid A \times\left\{y_{i}\right\}}$, et où les $y_{i}$ sont quelconques dans $\widehat{A}$. Nous n'avons toutefois pas jugé utile d'alourdir la présentation de cette proposition plus avant.

Démonstration. Fixons, pour $i=1, \ldots, 4$, des éléments $\left(a_{i}, l_{i}\right)$ de $K_{2} \times \widehat{K_{2}(4)}$, et notons $P$ le produit :

$$
P:=\Delta_{\left(a_{1}, l_{1}\right)}^{(2)}(s) \Delta_{\left(a_{2}, l_{2}\right)}^{(2)}(u) \Delta_{\left(a_{3}, l_{3}\right)}^{(2)}(v) \Delta_{\left(a_{4}, l_{4}\right)}^{(2)}(w) .
$$


En remplaçant les $\Delta_{\left(a_{i}, l_{i}\right)}^{(2)}$ par leur valeur (formule (8)), on a :

$$
P=\sum_{c_{1}, \ldots, c_{4} \in K(4)} \prod_{i=1}^{4} l_{i}\left(c_{i}\right) \delta_{a_{1}+c_{1}}^{(2)}(s) \delta_{a_{2}+c_{2}}^{(2)}(u) \delta_{a_{3}+c_{3}}^{(2)}(v) \delta_{a_{4}+c_{4}}^{(2)}(w)
$$

en tenant compte de la formule (3), appliquée une fois avec $X:=x+y$ et $Y:=z+t$ de sorte que $s=X+Y$ et $u=X-Y$, et une deuxième fois avec $X:=x-y$ et $Y:=z-t$ de sorte que $v=X+Y$ et $w=X-Y$, on obtient :

$$
\begin{aligned}
P= & \sum_{\substack{c_{1}, \ldots, c_{4} \in K(4) \\
\eta_{1}, \eta_{2} \in K(2)}} \prod_{i=1}^{4} l_{i}\left(c_{i}\right) \delta_{\frac{a_{1}+a_{2}+c_{1}+c_{2}}{2}+\eta_{1}}^{(3)}(x+y) \frac{\delta_{a_{1}-a_{2}+c_{1}-c_{2}}}{2}+\eta_{1} \\
& (z+t) \\
& \times \delta_{\frac{a_{3}+a_{4}+c_{3}+c_{4}}{2}+\eta_{2}}^{(3)}(x-y) \delta_{\frac{a_{3}-a_{4}+c_{3}-c_{4}}{2}+\eta_{2}}^{(3)}(z-t) .
\end{aligned}
$$

En appliquant une nouvelle fois la même formule (3), on en tire :

$$
\begin{aligned}
P= & \sum_{\substack{c_{1}, \ldots, c_{4} \in K(4) \\
\eta_{1}, \ldots, \eta_{4} \in K(2)}} \prod_{i=1}^{4} l_{i}\left(c_{i}\right) \delta_{\frac{a_{1}+a_{2}+a_{3}+a_{4}+c_{1}+c_{2}+c_{3}+c_{4}}{4}+\frac{\eta_{1}+\eta_{2}}{2}+\eta_{3}}^{(4)}(x) \\
& \times \delta_{\frac{a_{1}+a_{2}-a_{3}-a_{4}+c_{1}+c_{2}-c_{3}-c_{4}}{4}+\frac{\eta_{1}-\eta_{2}}{2}+\eta_{3}}^{(4)}(y) \\
& \times \delta_{\frac{a_{1}-a_{2}+a_{3}-a_{4}}{4}+\frac{c_{1}-c_{2}+c_{3}-c_{4}}{4}+\frac{\eta_{1}+\eta_{2}}{2}+\eta_{4}}^{(4)}(z) \\
& \times \delta_{\frac{a_{1}-a_{2}-a_{3}+a_{4}}{4}+\frac{c_{1}-c_{2}-c_{3}+c_{4}}{4}+\frac{\eta_{1}-\eta_{2}}{2}+\eta_{4}}^{(4)}(t) .
\end{aligned}
$$

En tenant compte du fait 3.3, point (i), on a :

$$
\delta_{\frac{\eta_{1}+\eta_{2}}{2}+\eta_{3}+\sum_{i=1}^{4} \frac{a_{i}+c_{i}}{4}}^{(4)}(x)=\frac{1}{8^{g}} \sum_{\alpha_{1} \in \widehat{K_{4}(8)}} \Delta_{\left(\frac{\eta_{1}+\eta_{2}}{2}+\eta_{3}+\sum_{i=1}^{4} \frac{a_{i}+c_{i}}{4}, \alpha_{1}\right)}^{(4)}(x),
$$

et de même pour les autres facteurs intervenant dans le produit ci-dessus.

Posons maintenant $d=c_{1}+c_{2}+c_{3}+c_{4}$ et remplaçons dans les expressions ci-dessus $c_{4}$ par $d-c_{1}-c_{2}-c_{3}$; on note que $\frac{c_{1}+c_{2}}{2}, \frac{c_{1}+c_{3}}{2}$ et $\frac{c_{2}+c_{3}}{2}$ sont des éléments de $K_{4}(8)$, et l'on applique le fait 3.3 , point (iv). On obtient donc :

$$
\Delta_{\left(\frac{d+b_{1}}{4}+\frac{\eta_{1}+\eta_{2}}{2}+\eta_{3}, \alpha_{1}\right)}^{(4)}(x)=\Delta_{\left(\frac{b_{1}+d}{4}, \alpha_{1}\right)}^{(4)}(x) \alpha_{1}^{-1}\left(\frac{\eta_{1}+\eta_{2}}{2}+\eta_{3}\right)
$$


et, de même :

$$
\left\{\begin{array}{l}
\Delta_{\left(\frac{b_{2}+2 c_{1}+2 c_{2}-d}{4}+\frac{\eta_{1}-\eta_{2}}{2}+\eta_{3}, \alpha_{2}\right)}^{(4)}(y)=\Delta_{\left(\frac{b_{2}-d}{4}, \alpha_{2}\right)}^{(4)}(y) \alpha_{2}^{-1}\left(\frac{c_{1}+c_{2}}{2}+\frac{\eta_{1}-\eta_{2}}{2}+\eta_{3}\right), \\
\Delta_{\left(\frac{b_{3}+2 c_{1}+2 c_{3}-d}{4}+\frac{\eta_{1}+\eta_{2}}{2}+\eta_{4}, \alpha_{3}\right)}^{(4)}(z)=\Delta_{\left(\frac{b_{3}-d}{4}, \alpha_{3}\right)}^{(4)}(z) \alpha_{3}^{-1}\left(\frac{c_{1}+c_{3}}{2}+\frac{\eta_{1}+\eta_{2}}{2}+\eta_{4}\right), \\
\Delta_{\left(\frac{b_{4}-2 c_{2}-2 c_{3}+d}{4}+\frac{\eta_{1}-\eta_{2}}{2}+\eta_{4}, \alpha_{4}\right)}^{(4)}(t)=\Delta_{\left(\frac{b_{4}+d}{4}, \alpha_{4}\right)}^{(4)}(t) \alpha_{4}^{-1}\left(-\frac{c_{2}+c_{3}}{2}+\frac{\eta_{1}-\eta_{2}}{2}+\eta_{4}\right) .
\end{array}\right.
$$

Notons maintenant :

$$
\begin{aligned}
F\left(\alpha_{1}, \ldots, \alpha_{4}\right)= & \sum_{\substack{c_{1}, c_{2}, c_{3} \in K(4) \\
\eta_{1}, \ldots, \eta_{4} \in K(2)}}\left(l_{1} \cdot l_{4}^{-1}\right)\left(c_{1}\right)\left(l_{2} \cdot l_{4}^{-1}\right)\left(c_{2}\right)\left(l_{3} \cdot l_{4}^{-1}\right)\left(c_{3}\right) \\
& \times \alpha_{1}^{-1}\left(\frac{\eta_{1}+\eta_{2}}{2}+\eta_{3}\right) \alpha_{2}^{-1}\left(\frac{c_{1}+c_{2}}{2}+\frac{\eta_{1}-\eta_{2}}{2}+\eta_{3}\right) \\
& \times \alpha_{3}^{-1}\left(\frac{c_{1}+c_{3}}{2}+\frac{\eta_{1}+\eta_{2}}{2}+\eta_{4}\right) \alpha_{4}^{-1}\left(-\frac{c_{2}+c_{3}}{2}+\frac{\eta_{1}-\eta_{2}}{2}+\eta_{4}\right),
\end{aligned}
$$

nous allons simplifier la fonction $F$ avant de la réinjecter dans l'expression trouvée pour $P$.

Tout d'abord, en isolant la somme

$$
\sum_{\eta_{4} \in K(2)} \alpha_{3}^{-1}\left(\frac{c_{1}+c_{3}}{2}+\frac{\eta_{1}+\eta_{2}}{2}+\eta_{4}\right) \alpha_{4}\left(\frac{c_{2}+c_{3}}{2}+\frac{\eta_{2}-\eta_{1}}{2}+\eta_{4}\right)
$$

la formule des caractères nous assure que cette somme ne peut être non nulle que si $\alpha_{4}=\alpha_{3} \bmod (\widehat{K(2)})$. De même, en isolant la somme portant sur $\eta_{3}$, on trouve $\alpha_{2}=\alpha_{1} \bmod (\widehat{K(2)})$; en isolant ensuite les sommes portant sur $\eta_{1}, \eta_{2}$, on trouve également que $\alpha_{3}=\alpha_{1} \bmod (\widehat{K(2)})$. En d'autres termes, les $\alpha_{i}$ sont tous égaux modulo des carrés, et nous pouvons donc poser $\alpha_{i}=\alpha_{1} \cdot\left(2 \star \beta_{i}\right)$, pour $i=2,3$ et 4 , où les $\beta_{i}$ sont des éléments de $\widehat{K_{2}(4)}$.

Isolons maintenant la somme portant sur $c_{2}$, c'est-à-dire :

$$
\sum_{c_{2} \in K_{2}(4)}\left(l_{2} \cdot l_{4}^{-1}\right)\left(c_{2}\right) \alpha_{1}^{-1}\left(\frac{c_{2}}{2}\right) 2 \star \beta_{2}^{-1}\left(\frac{c_{2}}{2}\right) \alpha_{1}\left(\frac{c_{2}}{2}\right) 2 \star \beta_{4}\left(\frac{c_{2}}{2}\right) ;
$$

on vérifie alors à l'aide de la formule des caractères, que cette somme ne peut être non nulle que si $\beta_{2}=l_{2} \cdot l_{4}^{-1} \cdot \beta_{4}$.

De même, en isolant la somme portant sur $c_{3}$, on trouve $\beta_{3}=\beta_{4} \cdot l_{3} \cdot l_{4}^{-1}$.

Sachant que l'expression $F$ ne peut être non nulle que sous ces conditions, 
celle-ci se simplifie alors en :

$$
\begin{aligned}
F\left(\alpha_{1}, \alpha_{1} \cdot 2 \star l_{2} \cdot 2 \star l_{4}^{-1} \cdot 2 \star \beta_{4}, \alpha_{1} \cdot 2 \star l_{3} \cdot 2 \star l_{4}^{-1} \cdot 2 \star \beta_{4}, \alpha_{1} \cdot 2 \star \beta_{4}\right) \\
=64^{g} \sum_{\substack{c_{1} \in K(4) \\
\eta_{1}, \eta_{2} \in K(2)}}\left(l_{1} \cdot l_{4} \cdot l_{2}^{-1} \cdot l_{3}^{-1}\right)\left(c_{1}\right) \cdot l_{2}\left(-\eta_{1}+\eta_{2}\right) \\
\quad \times l_{3}\left(-\eta_{1}-\eta_{2}\right) \cdot \alpha_{1}\left(-c_{1}\right) \cdot \beta_{4}\left(-2 c_{1}+\eta_{1}+\eta_{2}\right) .
\end{aligned}
$$

En isolant le terme en $\eta_{1}$ par exemple, on obtient la somme :

$$
\sum_{\eta_{1} \in K(2)} l_{2}\left(\eta_{1}\right) l_{3}\left(\eta_{1}\right) \beta_{4}\left(\eta_{1}\right)
$$

qui ne peut être non nulle que si $\beta_{4}=l_{2} \cdot l_{3} \cdot 2 \star \alpha$, avec $\alpha \in \widehat{K(2)}$, et l'on obtient (notons que la somme portant sur $\eta_{2}$ se simplifie également sous cette condition) :

$$
\begin{array}{r}
F\left(\alpha_{1}, \alpha_{1} \cdot 2 \star\left(l_{2}^{2} \cdot l_{4}^{-1} \cdot l_{3} \cdot 2 \star \alpha\right), \alpha_{1} \cdot 2 \star\left(l_{3}^{2} \cdot l_{4}^{-1} \cdot l_{2} \cdot 2 \star \alpha\right), \alpha_{1} \cdot 2 \star\left(l_{2} \cdot l_{3} \cdot 2 \star \alpha\right)\right) \\
=256^{g} \sum_{c_{1} \in K(4)} l_{1} \cdot l_{4} \cdot l_{2} \cdot l_{3}\left(c_{1}\right) \alpha_{1}\left(-c_{1}\right) .
\end{array}
$$

Notons, pour $i=1, \ldots, 4, \tilde{l}_{i}$ un relevé quelconque de $l_{i}$ dans $\widehat{K_{4}(8)}$, que nous fixerons une fois pour toutes. La formule des caractères nous assure que cette expression ne peut être non nulle que si $\alpha_{1}=\tilde{l}_{1} \cdot \tilde{l}_{2} \cdot \tilde{l}_{3} \cdot \tilde{l}_{4} \cdot 4 \star \gamma_{1}=\tilde{k}_{1} \cdot 4 \star \gamma_{1}$, où $\gamma_{1}$ est un élément de $\widehat{K(2)}$. On pose :

$$
4 \star \gamma_{2}=4 \star \gamma_{3}=4 \star \gamma_{4}=4 \star \gamma_{1} \cdot 2 \star\left(l_{2}^{2} \cdot l_{3}^{2} \cdot 2 \star \alpha\right) .
$$

Calculons avec ces notations et en notant que pour tout $i$ compris entre 1 et 4 on a, par définition, la relation $2 \star l_{i}=\tilde{l}_{i}^{2}$ :

$$
\begin{aligned}
\alpha_{1} \cdot 2 \star\left(l_{2}^{2} \cdot l_{4}^{-1} \cdot l_{3} \cdot 2 \star \alpha\right) & =4 \star \gamma_{1} \cdot 4 \star \alpha \cdot \tilde{l}_{1} \cdot\left(\tilde{l}_{2} \cdot 2 \star l_{2}^{2}\right) \cdot\left(\tilde{l}_{3} \cdot 2 \star l_{3}\right) \cdot\left(\tilde{l}_{4} \cdot 2 \star l_{4}^{-1}\right) \\
& =4 \star \gamma_{1} \cdot 4 \star \alpha \cdot \tilde{l}_{1} \cdot \tilde{l}_{2} \cdot \tilde{l}_{3}^{-1} \cdot \tilde{l}_{4}^{-1} \cdot 2 \star l_{2}^{2} \cdot 2 \star l_{3}^{2} \\
& =\tilde{k}_{2} \cdot 4 \star \gamma_{2} .
\end{aligned}
$$

On vérifie à l'aide d'un calcul similaire :

$$
\left\{\begin{array}{l}
\alpha_{1}=\tilde{k}_{1} \cdot 4 \star \gamma_{1}, \\
\alpha_{2}=\alpha_{1} \cdot 2 \star\left(l_{2}^{2} \cdot l_{4}^{-1} \cdot l_{3} \cdot 2 \star \alpha\right)=\tilde{k}_{2} \cdot 4 \star \gamma_{2}, \\
\alpha_{3}=\alpha_{1} \cdot 2 \star\left(l_{3}^{2} \cdot l_{4}^{-1} \cdot l_{2} \cdot 2 \star \alpha\right)=\tilde{k}_{3} \cdot 4 \star \gamma_{3}, \\
\alpha_{4}=\alpha_{1} \cdot 2 \star\left(l_{2} \cdot l_{3} \cdot 2 \star \alpha\right)=\tilde{k}_{4} \cdot 4 \star \gamma_{4} .
\end{array}\right.
$$

On en déduit, pour finir, en reportant dans $F$ :

$$
F\left(\tilde{k}_{1} \cdot 4 \star \gamma_{1}, \tilde{k}_{2} \cdot 4 \star \gamma_{2}, \tilde{k}_{3} \cdot 4 \star \gamma_{3}, \tilde{k}_{4} \cdot 4 \star \gamma_{4}\right)=1024^{g} .
$$


Il suffit maintenant de reporter ce calcul dans la valeur de $P$ que nous avions trouvée ci-dessus :

$$
\begin{aligned}
P= & \frac{1}{4^{g}} \sum_{\substack{d \in K(4) \\
\gamma_{1}, \alpha \in \widehat{K(2)}}} l_{4}(d) \Delta_{\left(\frac{b_{1}+d}{4}, \tilde{k}_{1} \cdot 4 \star \gamma_{1}\right)}^{(4)}(x) \Delta_{\left(\frac{b_{2}-d}{4}, \tilde{k}_{2} \cdot 4 \star \gamma_{2}\right)}^{(4)}(y) \\
& \times \Delta_{\left(\frac{b_{3}-d}{4}, \tilde{k}_{3} \cdot 4 \star \gamma_{3}\right)}^{(4)}(z) \Delta_{\left(\frac{b_{4}+d}{4}, \tilde{k}_{4} \cdot 4 \star \gamma_{4}\right)}^{(4)}(t) .
\end{aligned}
$$

En injectant le point (iv) du fait 3.3 (i. e. on remplace les termes $\frac{-d}{4}$ par $\frac{d}{4}$ en écrivant $\frac{-d}{4}=\frac{d}{4}-\frac{d}{2}$ ), et en tenant compte du fait que tous les $4 \star \gamma_{i}$ sont triviaux sur les points de $K(4)$, ceci donne :

$$
\begin{aligned}
P= & \frac{1}{4^{g}} \sum_{\substack{d \in K(4) \\
\alpha, \gamma_{1} \in \widehat{K(2)}}} l_{1}(d) \Delta_{\left(\frac{b_{1}+d}{4}, \tilde{k}_{1} \cdot 4 \star \gamma_{1}\right)}^{(4)}(x) \Delta_{\left(\frac{b_{2}+d}{4}, \tilde{k}_{2} \cdot 4 \star \gamma_{2}\right)}^{(4)}(y) \\
& \times \Delta_{\left(\frac{b_{3}+d}{4}, \tilde{k}_{3} \cdot 4 \star \gamma_{3}\right)}^{(4)}(z) \Delta_{\left(\frac{b_{4}+d}{4}, \tilde{k}_{4} \cdot 4 \star \gamma_{4}\right)}^{(4)}(t) .
\end{aligned}
$$

Notons maintenant $\mathcal{Z}$ un système de représentants de $K(4) / K(2)$. On a donc, toujours en tenant compte du point (iv) du fait 3.3 :

$$
\begin{aligned}
P= & \frac{1}{4^{g}} \sum_{\substack{d \in \mathcal{Z}, e \in K(2) \\
\alpha, \gamma_{1} \in \widehat{K(2)}}} l_{1}(d+e) \Delta_{\left(\frac{b_{1}+d+e}{4}, \tilde{k}_{1} \cdot 4 \star \gamma_{1}\right)}^{(4)}(x) \Delta_{\left(\frac{b_{2}+d+e}{4}, \tilde{k}_{2} \cdot 4 \star \gamma_{2}\right)}^{(4)}(y) \\
& \times \Delta_{\left(\frac{b_{3}+d+e}{4}, \tilde{k}_{3} \cdot 4 \star \gamma_{3}\right)}^{(4)}(z) \Delta_{\left(\frac{b_{4}+d+e}{4}, \tilde{k}_{4} \cdot 4 \star \gamma_{4}\right)}^{(4)}(t) \\
= & \frac{1}{4^{g}} \sum_{\alpha \in \mathcal{Z}} l_{1}(d) \Delta_{\left(\frac{b_{1}+d}{4}, \tilde{k}_{1} \cdot 4 \star \gamma_{1}\right)}^{(4)}(x) \Delta_{\left(\frac{b_{2}+d}{4}, \tilde{k}_{2} \cdot 4 \star \gamma_{2}\right)}^{(4)}(y) \\
& \times \Delta_{\left(\frac{b_{3}+d}{4}, \tilde{k}_{3} \cdot 4 \star \gamma_{3}\right)}^{(4)}(z) \Delta_{\left(\frac{b_{4}+d}{4}, \tilde{k}_{4} \cdot 4 \star \gamma_{4}\right)}^{(4)}(t) \sum_{e \in K(2)}^{(2)} l_{1}(e) \prod_{i=1}^{4}\left(\tilde{k}_{i} \cdot 4 \star \gamma_{i}\right)\left(\frac{-e}{4}\right) .
\end{aligned}
$$

Comme d'une part $\prod_{i=1}^{4} \tilde{k}_{i}=\tilde{l}_{1}^{4}$, et d'autre part

$$
\prod_{i=1}^{4} 4 \star \gamma_{i}=4 \star \gamma_{1}^{4} \cdot\left(2 \star\left(l_{2}^{6} \cdot l_{3}^{6} \cdot 2 \star \alpha^{3}\right)\right)=2 \star l_{2}^{2} \cdot 2 \star l_{3}^{2} \cdot 4 \star \alpha,
$$

on a

$$
\sum_{e \in K(2)} l_{1}(e) \prod_{i=1}^{4}\left(\tilde{k}_{i} \cdot 4 \star \gamma_{i}\right)\left(\frac{-e}{4}\right)=\sum_{e \in K(2)}\left(l_{1} \cdot \tilde{l}_{1} \cdot \alpha \cdot l_{2} \cdot l_{3}\right)(e)=\sum_{e \in K(2)}\left(\alpha \cdot l_{2} \cdot l_{3}\right)(e) .
$$

La formule des caractères nous donne donc que $\bar{l}_{2} \cdot \bar{l}_{3}=\alpha$ (rappelons que désigne ici la projection naturelle de $\widehat{K_{2}(4)}$ vers $\left.\widehat{K_{2}(2)}\right)$. En reportant la valeur de 
$\alpha$ que nous venons de calculer dans la définition des $\gamma_{i}$, on en tire :

$$
4 \star \gamma_{1}=4 \star \gamma_{2}=4 \star \gamma_{3}=4 \star \gamma_{4} .
$$

Cette simplification nous permet enfin de réécrire l'expression $P$ ci-dessus sous la forme voulue :

$$
\begin{aligned}
P= & \frac{1}{2^{g}} \sum_{\substack{d \in K(4) / K(2) \\
u \in \widehat{K(2)}}} l_{1}(d) \Delta_{\left(\frac{b_{1}+d}{4}, \tilde{k}_{1} \cdot 4 \star u\right)}^{(4)}(x) \Delta_{\left(\frac{b_{2}+d}{4}, \tilde{k}_{2} \cdot 4 \star u\right)}^{(4)}(y) \\
& \times \Delta_{\left(\frac{b_{3}+d}{4}, \tilde{k}_{3} \cdot 4 \star u\right)}^{(4)}(z) \Delta_{\left(\frac{b_{4}+d}{4}, \tilde{k}_{4} \cdot 4 \star u\right)}^{(4)}(t)
\end{aligned}
$$

(maintenant le membre de droite est invariant lorsque $d$ est inchangé modulo $K(2)$, ce qui justifie le fait de remplacer $\mathcal{Z}$ par $K(4) / K(2))$.

Ceci montre bien la proposition 3.6.

On peut maintenant énoncer les formules d'addition :

Proposition 3.7. Soient $(a, l),(b, k)$ des éléments quelconques de $K_{2} \times \widehat{K(4)}$. Choisissons des éléments $\left(a^{\prime}, l^{\prime}\right),\left(b^{\prime}, k^{\prime}\right)$ de $K_{2} \times \widehat{K(4)}$ tels que $\theta_{\mathcal{L}^{\otimes 4}}\left(a^{\prime}, l^{\prime}\right)$ et $\theta_{\mathcal{L} \otimes 4}\left(b^{\prime}, k^{\prime}\right)$ soient non nuls et tels que $a^{\prime}=a \bmod \left(2 K_{2}\right), b^{\prime}=b \bmod \left(2 K_{2}\right)$ et enfin $l^{\prime}=l \cdot \kappa^{2}, k^{\prime}=k \cdot \kappa^{\prime 2}$ pour certains éléments $\kappa, \kappa^{\prime}$ de $\widehat{K(4)}$. Posons $c_{1}=a+a^{\prime}+b+b^{\prime}$, $c_{2}=a+b-a^{\prime}-b^{\prime}, c_{3}=a-b+a^{\prime}-b^{\prime}$ et $c_{4}=a-b-a^{\prime}+b^{\prime} d^{\prime}$ une part et $l_{1}=l \cdot k \cdot \kappa \cdot \kappa^{\prime}$, $l_{2}=\kappa^{-1} \cdot \kappa^{\prime-1}, l_{3}=l \cdot k^{-1} \cdot \kappa \cdot \kappa^{\prime-1}$ et $l_{4}=\kappa^{-1} \cdot \kappa^{\prime}$ d'autre part. On a alors :

$$
\begin{aligned}
& 2^{g} \theta_{\mathcal{L}^{\otimes 4}}\left(a^{\prime}, l^{\prime}\right) \theta_{\mathcal{L} \otimes 4}\left(b^{\prime}, k^{\prime}\right) \Delta_{(a, l)}^{(2)}(x+y) \Delta_{(b, k)}^{(2)}(x-y)= \\
& \sum_{\substack{d \in K(4) / K(2) \\
u \in \widehat{K(2)}}} l(d) \Delta_{\left(\frac{c_{1}+d}{2}, l_{1} \cdot 2 \star u\right)}^{(2)}(x) \Delta_{\left(\frac{c_{2}+d}{2}, l_{2} \cdot 2 \star u\right)}^{(2)}(x) \Delta_{\left(\frac{c_{3}+d}{2}, l_{3} \cdot 2 \star u\right)}^{(2)}(y) \Delta_{\left(\frac{c_{4}+d}{2}, l_{4} \cdot 2 \star u\right)}^{(2)}(y),
\end{aligned}
$$

où $\frac{c_{i}+d}{2}$ pour $i=1,2,3,4$ sont choisis de sorte que leur somme vaille $2 a$.

En particulier, le morphisme $\xi$, défini à la formule (2), peut être représenté dans le plongement $\Theta_{\mathcal{L} \otimes 4}$ par des formes bi-homogènes ${ }^{8}$, de bi-degré $(2,2)$, à coefficients dans $^{9} \mathbb{Q}\left(\mu_{4}, \Theta_{\mathcal{L} \otimes 4}(0)\right)$ et la hauteur de Weil de la famille formée de 1 et de leurs coefficients est majorée par $2\left(4^{g}-1\right) h(A)$.

Démonstration. Tout d'abord, l'existence des éléments $\left(a^{\prime}, l^{\prime}\right),\left(b^{\prime}, k^{\prime}\right)$ est assurée par le lemme 3.5. On choisit pour commencer des relevés $\tilde{l}, \tilde{k}, \tilde{l}^{\prime}$ et $\tilde{k}^{\prime} \operatorname{de} l, k, l^{\prime}$ et $k^{\prime}$ dans $\widehat{K_{4}(8)}$ et l'on pose de même que ci-dessus $\tilde{m}_{1}=\tilde{l} \cdot \tilde{k} \cdot \tilde{l}^{\prime} \cdot \tilde{k}^{\prime}, \tilde{m}_{2}=\tilde{l} \cdot \tilde{k} \cdot \tilde{l}^{\prime-1} \cdot \tilde{k}^{\prime}{ }^{-1}$, $\tilde{m}_{3}=\tilde{l} \cdot \tilde{k}^{-1} \cdot \tilde{l}^{\prime} \cdot \tilde{k}^{\prime-1}$ et $\tilde{m}_{4}=\tilde{l} \cdot \tilde{k}^{-1} \cdot \tilde{l}^{-1} \cdot \tilde{k}^{\prime}$; il suffit d'appliquer la proposition 3.6,

\footnotetext{
8 Le fait que $\xi$ puisse être représenté par des formes de bi-degré $(2,2)$ est classique, confer par exemple [La-Ru], le point est plutôt dans le calcul de la hauteur.

9 Suivant l'usage, on note pour tout entier $n \geq 1, \mu_{n}$ le groupe des racines $n^{\text {ièmes }}$ de l'unité.
} 
en y remplaçant le quadruplet $(x, y, z, t)$ par $(x, x, y, y)$. On trouve ainsi :

$$
\begin{aligned}
& \Delta_{(a, l)}^{(2)}(2(x+y)) \Delta_{(b, k)}^{(2)}(2(x-y)) \theta_{\mathcal{L}^{\otimes 4}}\left(a^{\prime}, l^{\prime}\right) \theta_{\mathcal{L}^{\otimes 4}}\left(b^{\prime}, k^{\prime}\right) \\
&=\frac{1}{2^{g}} \sum_{\substack{d \in K(4) / K(2) \\
u \in \overline{K(2)}}} l(d) \Delta_{\left(\frac{c_{1}+d}{4}, \tilde{m}_{1} \cdot 4 \star u\right)}^{(4)}(x) \\
& \times \Delta_{\left(\frac{c_{2}+d}{4}, \tilde{m}_{2} \cdot 4 \star u\right)}^{(4)}(x) \Delta_{\left(\frac{c_{3}+d}{4}, \tilde{m}_{3} \cdot 4 \star u\right)}^{(4)}(y) \Delta_{\left(\frac{c_{4}+d}{4}, \tilde{m}_{4} \cdot 4 \star u\right)}^{(4)}(y) .
\end{aligned}
$$

Par hypothèse sur $a^{\prime}$ et $b^{\prime}$ les quantités $c_{1}, \ldots, c_{4}$ sont des éléments de $2 K_{2}$ et, de même, par hypothèse sur $l^{\prime}$ et $k^{\prime}$, les caractères $\tilde{m}_{i}, 1 \leq i \leq 4$, sont des carrés dans $\widehat{K_{4}(8)}$. On peut donc terminer la preuve en se ramenant de sections de $\mathcal{L}^{\otimes 16}$ à des sections de $\mathcal{L}^{\otimes 4}$ par le point (ii) du fait 3.3. On obtient alors :

$$
\begin{aligned}
& \Delta_{(a, l)}^{(2)}([2](x+y)) \Delta_{(b, k)}^{(2)}([2](x-y)) \theta_{\mathcal{L}^{\otimes 4}}\left(a^{\prime}, l^{\prime}\right) \theta_{\mathcal{L}^{\otimes 4}}\left(b^{\prime}, k^{\prime}\right) \\
& =\frac{1}{2^{g}} \sum_{\substack{d \in K(4) / K(2) \\
u \in \widehat{K(2)}}} l(d) \Delta_{\left(\frac{c_{1}+d}{2}, l \cdot k \cdot \kappa \cdot \kappa^{\prime} \cdot 2 \star u\right)}^{(2)}([2] x) \Delta_{\left(\frac{c_{2}+d}{2}, \kappa^{-1} \cdot \kappa^{\prime-1} \cdot 2 \star u\right)}^{(2)}([2] x) \\
& \quad \times \Delta_{\substack{\left(\frac{c_{3}+d}{2}, l \cdot k^{-1} \cdot \kappa \cdot \kappa^{\prime-1} \cdot 2 \star u\right) \\
(2)}}^{([2] y) \Delta_{\left(\frac{c_{4}+d}{2}, \kappa^{-1} \cdot \kappa^{\prime} \cdot 2 \star u\right)}^{(2)}([2] y) .}
\end{aligned}
$$

La proposition 3.7 suit en remarquant que $A$ est divisible; en effet, le supplément découle immédiatement de la formule que nous venons d'établir, par définition de la hauteur de WEIL, une fois noté d'une part que pour toute famille de nombres algébriques non nuls,

$$
h_{W}\left(1, x_{1}^{-1}, \ldots, x_{n}^{-1}\right) \leq n h_{W}\left(1, x_{1}, \ldots, x_{n}\right),
$$

et d'autre part, qu'il y a au plus $4^{g}$ éléments $(a, k)$ de $\left(K_{2} / 2 K_{2}\right) \times\left(\widehat{K_{2}(4)} /{\widehat{K_{2}(4)}}^{2}\right)$ tels que $\theta_{\mathcal{L} \otimes 4}(a, k) \neq 0$ (il est facile de vérifier directement sur les formules qu'il ne peut-y avoir de regroupement de termes). La proposition 3.7 est donc entièrement établie.

Passons maintenant aux formules de duplication :

Proposition 3.8. Soit $(a, l) \in K_{2} \times \widehat{K_{2}(4)}$; on a alors :

(i)

$$
\left[\Delta_{(a, l)}^{(2)}\right]^{4}=\frac{1}{2^{g}} \sum_{\substack{d \in K(4) / K(2) \\ u \in \widehat{K(2)}}} l(d) \theta_{\mathcal{L}^{\otimes 4}}\left(\frac{d}{2}, 2 \star u\right)^{3} \Delta_{\left(2 a+\frac{d}{2}, l^{2} \cdot 2 \star u\right)}^{(2)} \circ[2]
$$

où $\frac{d}{2}$ est un point de 2-division quelconque de $d$; 
(ii) pour tout couple $(b, k) \in K_{2} \times \widehat{K_{2}(4)}$ tel que $b=a \bmod \left(2 K_{2}\right)$ et $k=l \kappa^{2}$, pour un certain $\kappa \in \widehat{K_{2}(4)}$, on $a$ :

$$
\Delta_{(a, l)}^{(2)} \circ[2] \theta_{\mathcal{L} \otimes 4}(b, k)^{3}=\frac{1}{2^{g}} \sum_{\substack{d \in K(4) / K(2) \\ u \in \overline{K(2)}}} l(d) \Delta_{\left(\frac{a+3 b+d}{2}, l^{2} \cdot \kappa^{-1} \cdot 2 \star u\right)}^{(2)}\left[\Delta_{\left(\frac{a-b+d}{2}, \kappa^{-1} \cdot 2 \star u\right)}^{(2)}\right]^{3} .
$$

Démonstration. Commençons par (i) ; appliquons pour ce faire la proposition 3.6, avec $(x, y, z, t)=(x, 0,0,0)$. On obtient alors :

$$
\left[\Delta_{(a, l)}^{(2)}(x)\right]^{4}=\frac{1}{2^{g}} \sum_{\substack{d \in K(4) / K(2) \\ u \in \widehat{K(2)}}} l(d) \Delta_{\left(a+\frac{d}{4}, \tilde{l}^{4} \cdot 4 \star u\right)}^{(4)}(x) \theta_{\mathcal{L} \otimes 16}\left(\frac{d}{4}, 4 \star u\right)^{3} .
$$

Il suffit maintenant d'appliquer le fait 3.3, point (ii).

Passons maintenant au point (ii). Appliquons la proposition 3.6, avec cette fois $(x, y, z, t)=(x, x, x, x)$. On obtient :

$$
\begin{aligned}
\theta_{\mathcal{L}^{\otimes 4}}(b, k)^{3} \Delta_{(a, l)}^{(2)} \circ[4]= & \frac{1}{2^{g}} \sum_{\substack{d \in K(4) / K(2) \\
u \in \overline{K(2)}}} l(d) \Delta_{\left(\frac{a+3 b+d}{4}, \tilde{l} \cdot \tilde{k}^{3} \cdot 4 \star u\right)}^{(4)} \\
& \times\left[\Delta_{\left(\frac{a-b+d}{4}, \tilde{l} \cdot \tilde{k}^{-1} \cdot 4 \star u\right)}^{(4)}\right]^{3} .
\end{aligned}
$$

Cette fois encore, l'hypothèse nous permet d'appliquer le fait 3.3, point (ii), et l'on en tire :

$$
\begin{aligned}
\theta_{\mathcal{L}^{\otimes 4}}(b, k)^{3} \Delta_{(a, l)}^{(2)} \circ[2]= & \frac{1}{2^{g}} \sum_{\substack{d \in K(4) / K(2) \\
u \in \widehat{K(2)}}} l(d) \Delta_{\left(\frac{a+3 b+d}{2}, l^{2} \cdot \kappa^{-1} \cdot 2 \star u\right)}^{(2)} \\
\times & {\left[\Delta_{\left(\frac{a-b+d}{2}, \kappa^{-1} \cdot 2 \star u\right)}^{(2)}\right]^{3} . }
\end{aligned}
$$

Ce qui montre bien la proposition 3.8.

\subsection{Hauteurs normalisées sur les variétés abeliennes}

Soit $\boldsymbol{k}$ un corps de nombres contenant toutes les racines 4-ièmes de l'unité, que l'on supposera plongé dans $\mathbb{C}$, et $A$ une variété abelienne de dimension $g$, munie d'un fibré en droites ample et symétrique $\mathcal{M}$, définie sur $\boldsymbol{k}$. Rappelons qu'il est défini dans [Ph]-I une hauteur normalisée $\hat{h}$ des sous-variétés algébriques de $A$ satisfaisant un certain nombre de propriétés (voir proposition 9 de loc. cit.). Nous précisons la constante dans la comparaison de $\hat{h}$ et de la hauteur projective $h$ dans le plongement projectif $\Theta=\Theta_{\mathcal{L} \otimes 4}$ associé à la structure thêta décrite et fixée au 
sous-paragraphe 3.1 précédent pour le fibré $\mathcal{L}^{\otimes 4}=\mathcal{M}^{\otimes 16}$. On notera $D$ le degré du fibré $\mathcal{M}$.

Plus précisément, nous montrerons :

Proposition 3.9. Soit $V$ une sous-variété algébrique de $A$, définie sur $\overline{\mathbb{Q}}$. Alors,

$$
|\hat{h}(V)-h(V)| \leq c_{0}(\Theta) \cdot(\operatorname{dim}(V)+1) \cdot \operatorname{deg}(V),
$$

où $c_{0}(\Theta):=4^{g+1} h(A)+3 g \log (2)$.

Remarque. Cette constante est tout à fait comparable à celle obtenue pour les points par Y. MANIN et Y. ZARHIN (confer [Ma-Za]). Elle est un peu plus faible pour ce qui est de la dépendance en $h(A)$ (d'un facteur 4). Cette perte n'apparaît que lorsque l'on travaille avec des variétés de dimension $\geq 1$. Pour les points, le calcul redonnerait naturellement une dépendance en $\left(4^{g}-1\right) h(A)$, comme dans loc. cit. Pour ce qui est de la constante numérique finale par contre, la comparaison est plus lourde en raison des différences de normalisations (hauteur de WEIL dans loc. cit., hauteur projective ici, plongement associé à $\mathcal{M}^{\otimes 4}$ là bas et à $\mathcal{M}^{\otimes 16}$ ici). Tous calculs faits, notre estimation est très légèrement plus fine.

Avant de passer à la preuve de la proposition 3.9, nous donnons ci-dessous des versions effectives des lemmes intermédiaires de [Ph]-I, §. 3, concernant les comparaisons entre la hauteur projective et la hauteur normalisée. Nous nous permettrons donc, pour éviter trop de redites, ici ou là de reprendre des pans de preuves de cette référence; que les lecteurs préférant un texte plus « autoexplicite » veuillent bien nous en excuser.

Lemme 3.10. Soit $\xi$ un point de 2-torsion de $A$ et $V$ une sous-variété algébrique de $A$ définie sur $\overline{\mathbb{Q}} ;$ alors, $h(V)=h(\xi+V)$ et $d(V+\xi)=d(V)$.

Démonstration. (Remplace le lemme 4 de [Ph]-I.) On remarque simplement que la translation par un point de 2 torsion est donnée par une transformation linéaire de $\mathbb{P}^{N}$, de hauteur nulle. Plus précisément, on remarque que cette transformation linéaire est très particulière. C'est une action diagonale qui agit par multiplication par des racines de l'unité : cela résulte de la proposition 3.4 (dans le cas classique, cela se voit facilement sur les séries thêta, confer [Ig], pages 49-50); une telle transformation ne change donc pas les mesures locales de la hauteur projective (confer paragraphe 2) et laisse donc cette dernière invariante; il en est bien évidemment de même du degré.

Pour tout élément $(a, l) \in K_{2} \times \widehat{K(4)}$, fixons un élément $(b, k) \in K_{2} \times \widehat{K(4)}$, tel que $b=a \bmod \left(2 K_{2}\right)$ et $k=l . \kappa^{2}$ pour un certain $\kappa \in \widehat{K(4)}$ (on notera $\alpha(a, l)$ 
un tel choix) tel que $\theta_{\mathcal{L}^{\otimes 4}}(b, k) \neq 0$, et notons $G_{(a, l)}$ la forme

$$
\frac{1}{2^{g} \theta_{\mathcal{L}^{\otimes 4}}(b, k)^{3}} \sum_{\substack{d \in K(4) / K(2) \\ u \in \overline{K(2)}}} l(d) \Delta_{\left(\frac{a+3 b+d}{2}, l^{2} \cdot \kappa^{-1} \cdot 2 \star u\right)}^{(2)}\left(\Delta_{\left(\frac{a-b+d}{2}, \kappa^{-1} \cdot 2 \star u\right)}^{(2)}\right)^{3} ;
$$

l'existence d'un tel élément $(b, k)$ est assurée par le lemme 3.5. Notons également $\mathfrak{B}$ le point projectif

$$
\mathfrak{B}=\left(\cdots: \theta_{\mathcal{L}^{\otimes 4}}(b, k)^{-1}: \cdots\right)_{b \in K_{2} / 2 K_{2}, k \in \widehat{K}_{2}(4)} /{\widehat{K_{2}(4)}}^{2} .
$$

On vérifie aisément que $\mathfrak{B}$ est défini sur le même corps de nombres que l'origine $0_{A}=\Theta_{\mathcal{L}^{\otimes 4}}\left(0_{A}\right)$ (voir notation 3.2) de la variété abelienne $A$ et que (inégalité valable aussi bien pour la hauteur de WeIL que pour la hauteur projective) :

$$
h(\mathfrak{B}) \leq\left(4^{g}-1\right) h(A) .
$$

Avec ces notations on dispose du lemme :

Lemme 3.11. Soit $\boldsymbol{G}=\left(G_{(a, l)}\right)_{(a, l) \in K_{2} \times \widehat{K(4)}}$ le système de formes introduit cidessus; $\boldsymbol{G}$ représente les formules de duplication sur A, les formes $\left(G_{(a, l)}\right)$ sont de degré 4 , leurs coefficients sont dans $\mathbb{Q}\left(\mu_{4}, \mathfrak{B}\right) \subset \boldsymbol{k}$ et la hauteur de Weil de la famille formée de 1 et des coefficients de toutes les formes $\left(G_{(a, l)}\right)_{(a, l) \in K_{2} \times \widehat{K(4)}}$ est majorée par $3 h(\mathfrak{B})+g \log (2)$. Pour toute place $v$ de $\boldsymbol{k}$ et tout $\boldsymbol{x} \in A\left(\overline{\boldsymbol{k}}_{\boldsymbol{v}}\right)$, on dispose des inégalités :

$$
|2|_{v}^{g} \exp \left(-\delta_{v} g \log (4)-3 \log \left\|0_{A}\right\|_{v}\right) \leq \frac{\|\boldsymbol{G}(\boldsymbol{x})\|_{v}}{\|\boldsymbol{x}\|_{v}^{4}} \leq\left|\frac{1}{2}\right|_{v}^{g} \exp \left(\delta_{v} g \log (4)+3 \log \|\mathfrak{B}\|_{v}\right)
$$

où $\delta_{v}=1$ si $v \mid \infty$ et $\delta_{v}=0$ si v est finie. Plus généralement, pour tout entier $m \geq 1$ et en notant $\boldsymbol{G}^{(m)}$ les formules de multiplication par $2^{m}$, la hauteur de Weil de la famille formée de 1 et des coefficients de toutes les formes $\left(G_{(a, b)}^{(m)}\right)_{(a, l) \in K_{2} \times \widehat{K(4)}}$ est majorée par $4^{m} \cdot(h(\mathfrak{B})+g \log (2))$ et :

$$
|2|_{v}^{\frac{g\left(4^{m}-1\right)}{3}} C_{v}(\Theta)^{-4^{m}} \leq \frac{\left\|\boldsymbol{G}^{(m)}(\boldsymbol{x})\right\|_{v}}{\|\boldsymbol{x}\|_{v}^{4^{m}}} \leq\left|\frac{1}{2}\right|_{v}^{\frac{g\left(4^{m}-1\right)}{3}} C_{v}(\Theta)^{4^{m}}
$$

où $C_{v}(\Theta)=\exp \left(\delta_{v} \frac{g \log (4)}{3}+\log \|\mathfrak{B}\|_{v}+\log \left\|0_{A}\right\|_{v}\right)$.

Démonstration. Les formes $G_{(a, l)}$ représentent bien les formules de duplication (c'est la proposition 3.8, point (ii)) ; un calcul direct montre que la hauteur de la famille formée de 1 et des coefficients des formes $G_{(a, l)}$ est majorée par $3 h(\mathfrak{B})+$ $g \log (2)$ (on vérifie là encore sur la formule qu'il ne peut-y avoir de simplification). On majore la hauteur de la famille formée de 1 et des coefficients des formes $\left(G_{(a, b)}^{(m)}\right)_{(a, l) \in K_{2} \times \widehat{K(4)}}$ par récurrence à partir de l'estimation précédente; toutefois, 
à ce stade, vu la complexité des formules, il n'est pas clair qu'il n'y a pas de regroupement de termes; on tient donc compte de la longueur des formes pour obtenir la majoration annoncée.

Comme la deuxième série d'inégalités (12) s'obtient par récurrence à partir de l'encadrement (11), il suffit de montrer ce dernier; la majoration de (11) découle de la proposition 3.8, point (ii) ; la minoration est, elle, une conséquence de la proposition 3.8, point (i), tout au moins pour les places finies. Pour les places archimédiennes, les deux calculs étant tout à fait similaires, nous nous contenterons d'expliquer la majoration. Notons $I:=\mathcal{Z}_{2} \times \widehat{K_{2}(4)}, J:=K_{2} / 2 K_{2} \times{\widehat{K_{2}(4)}}_{\widehat{K}_{2}(4)}^{2}$. Il s'agit d'estimer : $\sum_{i \in I}\left|G_{i}(\boldsymbol{x})\right|^{2}$; le lemme 3.8 , point (ii) nous assure que

$$
4^{g} \sum_{i \in I}\left|G_{i}(\boldsymbol{x})\right|^{2} \leq \sum_{i \in I} \frac{1}{\left|\theta_{\mathcal{L}^{\otimes 4}}(i)\right|^{6}}\left(\sum_{u, v}\left|x_{u}\right| \cdot\left|x_{v}\right|^{3}\right)^{2},
$$

et donc :

$$
4^{g} \sum_{i \in I}\left|G_{i}(\boldsymbol{x})\right|^{2} \leq \sum_{i \in I} \frac{1}{\left|\theta_{\mathcal{L}^{\otimes 4}}(i)\right|^{6}}\left(\sum_{u}\left|x_{u}\right|^{2}\right)\left(\sum_{v}\left|x_{v}\right|^{6}\right),
$$

ou encore

$$
\begin{aligned}
4^{g} \sum_{i \in I}\left|G_{i}(\boldsymbol{x})\right|^{2} & \leq \sum_{i \in J} \frac{1}{\left|\theta_{\mathcal{L}^{\otimes 4}}(i)\right|^{6}} \sum_{I / J}\left(\sum_{u}\left|x_{u}\right|^{2}\right)\left(\sum_{v}\left|x_{v}\right|^{6}\right) \\
& \leq\left(\sum_{i \in J} \frac{1}{\left|\theta_{\mathcal{L} \otimes 4}(i)\right|^{12}}\right)^{1 / 2}\left(\sum_{J}\left(\sum_{I / J}\left(\sum_{u}\left|x_{u}\right|^{2}\right)\left(\sum_{v}\left|x_{v}\right|^{6}\right)\right)^{2}\right)^{1 / 2} \\
& \leq 16^{g}\|\mathfrak{B}\|^{6}\left(\sum_{I}\left|x_{u}\right|^{2}\right)\left(\sum_{I}\left|x_{v}\right|^{6}\right)
\end{aligned}
$$

où l'on observe pour la dernière inégalité que chaque terme intervenant dans ces sommes est répété $4^{g}$ fois. Au total, on a bien :

$$
\|\boldsymbol{G}(\boldsymbol{x})\| \leq 2^{g}\|\mathfrak{B}\|^{3} \cdot\|\boldsymbol{x}\|^{4} .
$$

Le lemme 3.11 est donc établi.

On notera que cet énoncé n'est rien d'autre qu'une version quantitative du fait 3, page 276 de $[\mathrm{Ph}]-\mathrm{I}$ pour $S=\{2\}$.

Lemme 3.12. Pour toute sous-variété algébrique $V$ de $A$, de dimension $r-1$, définie sur $\overline{\mathbb{Q}}$ et tout entier $m \geq 0$, on $a$ :

$$
\left|h(V)-\frac{h\left(\left[2^{m}\right]^{-1} V\right)}{4^{(g-r) m}}\right| \leq c_{1} r 4^{m} \operatorname{deg}(V),
$$

où $c_{1}=4^{g} h(A)+\frac{2}{3} g \log (2)$. 
Démonstration. On reprend la preuve du lemme 5 de $[\mathrm{Ph}]-\mathrm{I}$, mais ici $\sigma_{m}$ désigne le plongement de Veronese de degré $4^{m}$ de $\mathbb{P}_{N}$ dans $\mathbb{P}_{N^{\prime}}$ (où $N^{\prime}+1=\left({ }^{N+4^{m}}\right)$ ) et $f_{m}$ est une forme éliminante de $\sigma_{m}\left(\left[2^{m}\right]^{-1} V\right)$, d'indice $\left(4^{m}, \ldots, 4^{m}\right) \in \mathbb{N}^{r}$. La hauteur projective utilisée dans le présent texte (confer paragraphe 2) satisfait $h\left(f_{m}\right)=4^{m r} h\left(\left[2^{m}\right]^{-1} V\right)$ et $d^{\circ} f_{m}=r 4^{m(r-1)} \operatorname{deg}\left(\left[2^{m}\right]^{-1} V\right)=r 4^{m g} \operatorname{deg}(V)$. Et, si $\mu^{*}$ désigne comme dans loc. cit. la spécialisation des variables de $f_{m}$ en les coefficients de combinaisons génériques des formes $\left(G_{(a, l)}\right)_{(a, l) \in K_{2} \times \widehat{K(4)}}$ fournies par le lemme 3.11, $h\left(\mu^{*}\left(f_{m}\right)\right)=4^{m g} h(V)$. Finalement, on compare $h\left(f_{m}\right)$ et $h\left(\mu^{*}\left(f_{m}\right)\right)$ en décomposant les mesures locales $M_{v}\left(\mu^{*}\left(f_{m}\right) / f_{m}\right)$ par la formule de PoIsson comme dans la preuve du lemme 5 de loc. cit. et en utilisant les estimations du lemme 3.11 plutôt que le fait 3 de cette référence. On a ainsi

$$
\left.|2|\right|^{\frac{g\left(4^{m}-1\right)}{3} d^{\circ} f_{m}} C_{v}(\Theta)^{-4^{m} d^{\circ} f_{m}} \leq M_{v}\left(\frac{\mu^{*}\left(f_{m}\right)}{f_{m}}\right) \leq\left|\frac{1}{2}\right|_{v}^{\frac{g\left(4^{m}-1\right)}{3} d^{\circ} f_{m}} C_{v}(\Theta)^{4^{m} d^{\circ} f_{m}} .
$$

En sommant le logarithme de $M_{v}\left(\mu^{*}\left(f_{m}\right) / f_{m}\right)$ sur toutes les places d'un corps de définition de $f_{m}$

$$
\left|h\left(\mu^{*}\left(f_{m}\right)\right)-h\left(f_{m}\right)\right| \leq 4^{m} \cdot d^{\circ} f_{m} \sum_{v} \frac{\left[\boldsymbol{k}_{v}: \mathbb{Q}_{v}\right]}{[\boldsymbol{k}: \mathbb{Q}]} \log \left(C_{v}(\Theta)\right) .
$$

En combinant avec ce qui précède on obtient l'estimation annoncée avec

$$
\begin{aligned}
c_{1} & =\sum_{v} \frac{\left[\boldsymbol{k}_{v}: \mathbb{Q}_{v}\right]}{[\boldsymbol{k}: \mathbb{Q}]} \log \left(C_{v}(\Theta)\right) \\
& \leq \frac{g \log (4)}{3}+h(\mathfrak{B})+h\left(0_{A}\right) \\
& \leq 4^{g} h(A)+\frac{g \log (4)}{3} .
\end{aligned}
$$

D'où le lemme 3.12 .

Lemme 3.13. Avec les hypothèses du lemme 3.12 et en notant $S_{V}$ le stabilisateur de $V$ dans $A$, on a pour tout entier $m \geq 1$,

$$
\left|h\left(\left[2^{m}\right] V\right)-\frac{4^{r m} h(V)}{\left|\operatorname{ker}\left(\left[2^{m}\right]\right) \cap S_{V}\right|}\right| \leq c_{1} r \frac{4^{r m+1} \operatorname{deg}(V)}{\left|\operatorname{ker}\left(\left[2^{m}\right]\right) \cap S_{V}\right|} .
$$

Démonstration. (C'est celle des lemme 6, 7 et 8 de loc. cit.) On fait ici le calcul car un remplacement brutal de $C_{S}^{\prime \prime}$ par sa valeur nous ferait perdre un peu. On applique le lemme 3.12 à $W=\left[2^{m}\right] V$. L'expression

$$
\left[2^{m}\right]^{-1} W=\bigcup_{\xi \in \operatorname{ker}\left[2^{m}\right] / \operatorname{ker}\left[2^{m}\right] \cap S_{V}}(\xi+V)
$$


nous assure que :

$$
\left|h(W)-\sum_{\xi \in \operatorname{ker}\left[2^{m}\right] / \operatorname{ker}\left[2^{m}\right] \cap S_{V}} \frac{h(V+\xi)}{4^{m(g-r)}}\right| \leq c_{1} r \frac{4^{r m} \operatorname{deg}(V)}{\left|\operatorname{ker}\left(\left[2^{m}\right]\right) \cap S_{V}\right|} .
$$

Le lemme 3.10 donne maintenant :

$$
\sum_{\xi \in \operatorname{ker}[2] / \operatorname{ker}[2] \cap S_{V}} \frac{h(V+\xi)}{4^{g-r}}=\frac{4^{r} h(V)}{\left|\operatorname{ker}[2] \cap S_{V}\right|},
$$

combinant avec $(*)$ on en déduit le résultat souhaité pour $m=1$ avec $c_{1}$ remplacée par $c_{1} / 4$. Reprenant la preuve du lemme 7 de [Ph]-I on montre par récurrence sur $m^{\prime} \geq 1$ que pour tout point de $2^{\infty}$-torsion on a :

$$
|h(V+\xi)-h(V)| \leq 3 c_{1} r \operatorname{deg}(V) .
$$

Enfin, substituant ce dernier résultat au lemme 3.10 dans $(*)$ on obtient la conclusion recherchée.

Démonstration de la proposition 3.9. Le lemme 3.13 donne

$$
\left|\frac{h\left(\left[2^{m}\right] V\right)}{4^{m} \operatorname{deg}\left(\left[2^{m}\right] V\right)}-\frac{h(V)}{\operatorname{deg}(V)}\right| \leq 4 c_{1} r .
$$

Et comme

$$
\frac{\hat{h}(V)}{\operatorname{deg}(V)}=\lim _{m \rightarrow \infty} \frac{h\left(\left[2^{m}\right] V\right)}{4^{m} \operatorname{deg}\left(\left[2^{m}\right] V\right)},
$$

on en tire bien la proposition par passage à la limite (et en remplaçant $c_{1}$ par sa valeur).

Passons au plongement «enroulé » de [Ph]-III. Rappelons que ce dernier permet, dans certaines situations, par une technique de passage à la limite de se débarasser des constantes de comparaison entre les hauteurs projectives et hauteurs normalisées. Pour raffiner les estimations, nous utilisons ici le plongement «étiré » de [Da-Phi2] défini par :

$$
\begin{aligned}
\Phi_{\ell}: A & \hookrightarrow A^{2} \quad \stackrel{\Theta^{2}}{\longrightarrow}\left(\mathbb{P}_{N}\right)^{2} \stackrel{\text { SEGRE }}{\longrightarrow} \mathbb{P}_{N^{2}+2 N} \\
x & \longmapsto\left(x,\left[2^{\ell}\right] x\right) .
\end{aligned}
$$

Nous noterons $\operatorname{deg}_{\ell}, h_{\ell}$ (respectivement $\hat{h}_{\ell}$ ) le degré et la hauteur relative au plongement $\Phi_{\ell}$ (respectivement la hauteur normalisée). Nous reprenons ici les propositions 7 et 9 de loc. cit. pour $\Phi_{\ell}$ :

Proposition 3.14. Soit $V$ une sous-variété algébrique de $A$, définie sur $\overline{\mathbb{Q}}$ de dimension $r-1$. Alors :

$$
\left|\hat{h}_{\ell}(V)-h_{\ell}(V)\right| \leq 2 c_{0}(\Theta) r \operatorname{deg}_{\ell}(V) .
$$


Démonstration. On remarque que la démonstration de la proposition 3.9 ne fait intervenir le plongement $\Theta$ que par les propriétés des lemmes 3.11 et 3.10; comme ces dernières sont encore valables pour les plongements $\Phi_{\ell}$, quitte à remplacer $c_{1}$ par $2 c_{1}$, le résultat est établi.

Proposition 3.15. Soit $V$ une sous-variété algébrique de $A$ définie sur $\overline{\mathbb{Q}}$, de dimension $r-1$. Alors, avec les notations ci-dessus, on a

$$
\operatorname{deg}_{\ell}(V)=\left(4^{\ell}+1\right)^{r-1} \cdot \operatorname{deg}(V) \quad \text { et } \quad \hat{h}_{\ell}(V)=\left(4^{\ell}+1\right)^{r} \cdot \hat{h}(V) .
$$

En particulier, $\frac{h_{\ell}(V)}{4^{\ell} \cdot d_{\ell}(V)}$ tend vers $\frac{\hat{h}(V)}{d(V)}$ lorsque $\ell$ tend vers l'infini.

Démonstration. Nous suivons la preuve de la proposition $7 \mathrm{de}$ [Ph]-III. On obtient une forme de CHOw de $\Phi_{\ell}(V)$ en spécialisant les formes génériques de degrés $4^{\ell}+1$ en des combinaisons linéaires générales $\operatorname{des} X_{i} G_{j}^{(\ell)}(X)$ dans une forme éliminante d'indice $\left(4^{\ell}+1, \ldots, 4^{\ell}+1\right)$ de $V$. Le résultat s'en déduit en estimant les mesures de telles formes linéaires à l'aide du lemme 3.11 et en appliquant l'estimation obtenue aux variétés $\left[2^{m}\right] V$ lorsque $m \rightarrow \infty$.

\section{Minoration de hauteurs normalisées}

Nous allons établir ici le théorème 1.1; nous allons donc supposer donnée dans tout ce paragraphe, une variété abelienne $A$ de dimension $g \geq 1$, définie sur un corps de nombres $\boldsymbol{k}$, munie d'un fibré en droites ample et symétrique $\mathcal{M}$ associé à une polarisation principale. Nous supposerons que $A$ est plongée dans un espace projectif $\mathbb{P}_{N}$, avec $N=16^{g}-1$ via le plongement $\Theta=\Theta_{\mathcal{L}^{\otimes 4}}\left(\right.$ avec $\left.\mathcal{L}=\mathcal{M}^{\otimes 4}\right)$ décrit dans le paragraphe $\S .3 .1$; on dispose donc d'une comparaison numérique entre les hauteurs projectives et normalisées.

On munit $A \times A$ du fibré $\widetilde{\mathcal{M}}:=\pi_{1}^{\star} \mathcal{M} \otimes \pi_{2}^{\star} \mathcal{M}$, que l'on plonge dans $\mathbb{P}_{N^{\prime}}$ (avec $\left.N^{\prime}=(N+1)^{2}-1\right)$, via les coordonnées de MumFord associées à $\widetilde{\mathcal{M}}^{\otimes 16}$, ce qui revient à plonger $A \times A$ dans $\mathbb{P}_{N^{2}+2 N}$ par le plongement de SEGRE $\mathbb{P}_{N}^{2} \hookrightarrow \mathbb{P}_{N^{2}+2 N}$. Nous noterons $\Theta^{2}$ ce plongement.

À toute place archimédienne $v$ de $\boldsymbol{k}$ nous associons un plongement $\sigma_{v}$ de $\boldsymbol{k}$ dans $\mathbb{C}$ et nous identifierons $\sigma_{v}(A)(\mathbb{C})$ à un tore complexe $\mathbb{C}^{g} / \Lambda_{v}$. Nous disposons donc du plongement projectif

$$
\Theta_{v}: \sigma_{v}(A)(\mathbb{C}) \hookrightarrow \mathbb{P}_{n}(\mathbb{C}),
$$

où $\Theta_{v}$ est le plongement projectif associé à $\mathcal{M}_{\mathbb{C}_{v}}^{\otimes 16}$ décrit au paragraphe 3.1, et que l'on peut également sans perte de généralité ni modification des constantes de comparaisons entre hauteurs projectives et normalisées décrire à l'aide des fonctions thêta classiques (confer §. 3.1); on a $\Theta_{v} \circ \sigma_{v}(A)=\sigma_{v} \circ \Theta(A)$. Nous noterons $\Theta_{v}^{2}$ le plongement déduit de $\Theta_{v}$ pour $\sigma_{v}(A) \times \sigma_{v}(A)$. Nous désignerons par $H_{v}$ la 
forme de RiEmann associée à $\Theta_{v}$ et par $H_{v}^{2}$ la forme de RiEmann associée à $\Theta_{v}^{2}$. Nous noterons $T_{\sigma_{v}(A)(\mathbb{C})}$ l'espace tangent à l'origine de $\sigma_{v}(A)(\mathbb{C})$.

Pour tout entier $\ell \geq 1$, nous considérerons également le plongement étiré :

$$
\begin{aligned}
\Phi_{\ell}: A & \hookrightarrow A \times A \hookrightarrow \mathbb{P}_{N^{\prime}} \\
x & \longmapsto\left(x, 2^{\ell} x\right) .
\end{aligned}
$$

Nous noterons $\Phi_{\ell}^{2}$ le plongement étiré de $A \times A$.

Enfin, lorsqu'aucune ambiguïté sur la place archimédienne $v$ considérée ne nous semblera possible, nous omettrons les indices $v$ des objets introduits ci-dessus.

\subsection{Préliminaires}

La structure générale de la preuve est la même que dans [Da-Phi1]; nous la rappelons brièvement et donnons les valeurs numériques des diverses estimations qui nous seront utiles.

Soit $X$ une sous-variété algébrique de $A$, définie sur $\overline{\mathbb{Q}}$, qui n'est pas translatée d'une sous-variété abelienne de $A$. On note $B_{X}$ la sous-variété abelienne de $A$ engendrée par $X-X$ et $k_{X}$ désigne le nombre minimal de copies de $X-X$ dont la somme vaut $B_{X}$. On supposera pour commencer que $k_{X}=1$ (une récurrence permettra d'en déduire le cas général), et nous noterons $\boldsymbol{k}^{\prime}$ une extension finie de $\boldsymbol{k}$ sur laquelle $B_{X}$ est définie.

Décrivons la situation que nous allons utiliser. Introduisons l'isomorphisme:

$$
\begin{aligned}
& s: A^{2} \longrightarrow A^{2} \\
& \left(x_{1}, x_{2}\right) \longmapsto\left(x_{1}, x_{1}-x_{2}\right) .
\end{aligned}
$$

Rappelons que (voir par exemple [Ph]-III, proposition 1) :

$$
\operatorname{deg}\left(X^{2}\right)=\left(\begin{array}{c}
2 \operatorname{dim}(X) \\
\operatorname{dim}(X)
\end{array}\right) \cdot \operatorname{deg}(X)^{2} \quad \text { et } \hat{h}\left(X^{2}\right)=2\left(\begin{array}{c}
2 \operatorname{dim}(X)+1 \\
\operatorname{dim}(X)
\end{array}\right) \cdot \hat{h}(X) \operatorname{deg}(X) .
$$

Comme le plongement de $A$ dans $\mathbb{P}_{N}$ est projectivement normal (confer par exemple [Bi-La], chapitre 7, théorème 3.1, page 190), l'isomorphisme $s$ (ainsi que son inverse) peut être représenté par des formules bi-homogènes de bi-degré $(2,2)$ (confer proposition 3.7), et l'on a :

$$
\left\{\begin{array}{l}
2^{-2 \operatorname{dim}(X)} \operatorname{deg}\left(X^{2}\right) \leqslant \operatorname{deg}\left(s\left(X^{2}\right)\right) \leqslant 2^{2 \operatorname{dim} X} \operatorname{deg}\left(X^{2}\right) \\
2^{-2 \operatorname{dim}(X)-1} \cdot \hat{h}\left(X^{2}\right) \leqslant \hat{h}\left(s\left(X^{2}\right)\right) \leqslant 2^{2 \operatorname{dim}(X)+1} \cdot \hat{h}\left(X^{2}\right) .
\end{array}\right.
$$

Soit $\pi_{2}$ la projection linéaire de $\mathbb{P}_{N}^{2}$ sur le second facteur $\mathbb{P}_{N}$, on a alors $\pi_{2} \circ s\left(X^{2}\right)$ $=X-X$ et :

$$
\begin{aligned}
& \operatorname{deg}(X-X)=\operatorname{deg}\left(\pi_{2} \circ s\left(X^{2}\right)\right) \leqslant \operatorname{deg}\left(s\left(X^{2}\right)\right) \leqslant 16^{\operatorname{dim}(X)} \cdot \operatorname{deg}(X)^{2}, \\
& \hat{h}(X-X)=\hat{h}\left(\pi_{2} \circ s\left(X^{2}\right)\right) \leqslant \hat{h}\left(s\left(X^{2}\right)\right) \leqslant 8.16^{\operatorname{dim}(X)} \cdot \hat{h}(X) \operatorname{deg}(X) .
\end{aligned}
$$


On notera $B=B_{X}=X-X, W=s\left(X^{2}\right)$ et $\pi_{2}$ le morphisme surjectif

$$
\pi_{2}: W \longrightarrow B
$$

dont la fibre générique est de dimension $2 \operatorname{dim}(X)-\operatorname{dim}(B)$ et la fibre spéciale $W \cap \pi_{2}^{-1}(0)$ est de dimension $\operatorname{dim}(X)>2 \operatorname{dim}(X)-\operatorname{dim}(B)$. On notera que cette fibre spéciale s'identifie à $X \times\{0\}$ et est donc de degré $\operatorname{deg}(X)$.

Nous donnons tout d'abord une version quantitative du «lemme du volume » (lemme 4.3 de [Da-Phi1], § 4), qui permet de minorer la hauteur normalisée; afin de simplifier (et d'améliorer) les estimations numériques, nous commençons par établir la proposition suivante, qui permettra de «travailler en moyenne ».

Proposition 4.1. Soit $A=\mathbb{C}^{g} / \Lambda$ une variété abelienne complexe principalement polarisée, plongée dans $\mathbb{P}_{N}$ comme ci-dessus, et $\Omega_{\mathbb{P}_{N}}$ la forme de Fubini-Study sur $\mathbb{P}_{N}(\mathbb{C}), \tau_{x}$ la translation par $x$ dans $A$ et $d \nu_{A}$ la mesure de Haar normalisée sur A. Alors, pour $d=1, \ldots, g$ on a, en tout point $z$ de $A$,

$$
\int_{A}\left(\Theta \circ \tau_{x}\right)^{\star}\left(\Omega_{\mathbb{P}_{N}}^{\wedge d}\right)(z) \wedge d \nu_{A}(x)=\left(\pi d d^{c} H(z, z)\right)^{\wedge d}
$$

et $\pi d d^{c} H(z, z)=\frac{H(d z, d z)}{-2 \mathrm{i}}$.

Démonstration. On pose $f:=\log \|\Theta\|^{2}-\pi H$, où $\|\cdot\|$ est la norme euclidienne; c'est une fonction périodique par rapport à $\Lambda$ (confer [Ig], lemma 4, page 69) et l'intégrant du membre de gauche s'écrit alors

$$
\left(d_{z} d_{z}^{c} \log \|\Theta(z+x)\|^{2}\right)^{\wedge d}=\left(\pi d_{z} d_{z}^{c} H(z+x, z+x)\right)^{\wedge d}+\sum_{k=1}^{d}\left(\begin{array}{l}
d \\
k
\end{array}\right) \cdot d_{z} \omega_{k}(z, x)
$$

avec

$$
\omega_{k}(z, x):=d_{z}^{c} f(z+x) \wedge\left(d_{z} d_{z}^{c} f(z+x)\right)^{\wedge(k-1)} \wedge\left(\pi d_{z} d_{z}^{c} H(z+x, z+x)\right)^{\wedge(d-k)}
$$

pour $k=1, \ldots, d$. Comme $d_{z} d_{z}^{c} H$ est invariante par translations,

$$
\int_{A}\left(\pi d_{z} d_{z}^{c} H(z+x, z+x)\right)^{\wedge d} d \nu_{A}(x)=\left(\pi d d^{c} H(z, z)\right)^{\wedge d} .
$$

Il suffit de montrer que pour $k=1, \ldots, d$, les composantes de la $(d, d)$-forme $\int_{A} d_{z} \omega_{k}(z, x) \wedge d \nu_{A}(x)$ sont nulles. Pour $I, J \subset\{1, \ldots, g\}$ de même cardinal $m$ et ordonnés de façon croissante, notons $\mu_{I, J}(z)$ la $(m, m)$-forme $\frac{d z_{i_{1}} \wedge d \bar{z}_{j_{1}} \wedge \cdots \wedge d z_{i_{m}} \wedge d \bar{z}_{j_{m}}}{(-2 \mathrm{i})^{m}}$, on vérifie la relation $d \nu_{A}(x)=\operatorname{det}(H) \cdot \mu_{I_{g}, I_{g}}(x)$ où $I_{g}=\{1, \ldots, g\}$ et, pour $I, J$ de cardinal $g-d$, que la forme

$$
d_{z} \omega_{k}(z, x) \wedge d \nu_{A}(x) \wedge \mu_{I, J}(z)=d_{x} \omega_{k}(x, z) \wedge \mu_{I, J}(x) \wedge d \nu_{A}(z)
$$

est fermée pour $d_{x}$.

Pour conclure, on note que $\omega_{k}(x, z)$ est périodique par rapport à $\Lambda$; on déduit donc de la formule de STOKES que l'intégrale de $d_{z} \omega_{k}(z, x) \wedge d \nu_{A}(x) \wedge \mu_{I, J}(z)$ sur 
$A$ est nulle. Ceci, établi pour tous $I, J$ de cardinal $g-d$, montre que les formes $\int_{A} d_{z} \omega_{k}(z, x) \wedge d \nu_{A}(x)$ sont nulles pour $k=1, \ldots d$, d'où la proposition.

Lemme 4.2. Soit $W$ une sous-variété de $A^{2}, \Omega_{\mathbb{P}_{N^{\prime}}^{2}}$ la forme de Fubini-Study sur $\mathbb{P}_{N^{\prime}}^{2}, \exp _{A}$ l'application exponentielle de $A$ et $B_{r}=\left\{z \in T_{A(\mathbb{C})} ; \pi H(z, z) \leqslant r^{2}\right\}$. Si $r<\min \left\{\frac{\sqrt{\pi H(\lambda, \lambda)}}{2} ; \lambda \in \Lambda \backslash\{0\}\right\}$ et $\pi_{2}$ désigne la projection de $A^{2}$ sur le second facteur alors,

$$
\int_{A^{2}} \int_{W \cap \pi_{2}^{-1}\left(\exp _{A}\left(B_{r}\right)\right)}\left(\Phi_{\ell}^{2} \circ \tau_{x}\right)^{\star}\left(\Omega_{\mathbb{P}_{N^{\prime}}^{2}}^{\wedge \operatorname{dim}(W)}\right) \wedge d \nu_{A^{2}}(x)
$$

est minoré par :

$$
\left(4^{\ell}+1\right)^{\operatorname{dim}(W)} \cdot \operatorname{deg}\left(W \cap \pi_{2}^{-1}(0)\right) \cdot r^{2\left(\operatorname{dim}(W)-\operatorname{dim}\left(W \cap \pi_{2}^{-1}(0)\right)\right)} .
$$

Démonstration. Notons $\mathcal{D}$ un domaine fondamental de $\mathbb{C}^{g} / \Lambda$; la proposition 4.1 qui précède permet d'écrire l'intégrale considérée sous la forme

$$
\begin{aligned}
\mathcal{V} & =\int_{\exp _{A^{2}}^{-1}(W) \cap\left(\mathcal{D} \times B_{r}\right)}\left(\pi d d^{c} H^{2}(z, z)+\pi d d^{c} H^{2}\left(2^{\ell} z, 2^{\ell} z\right)\right)^{\wedge \operatorname{dim}(W)} \\
& =\left(4^{\ell}+1\right)^{\operatorname{dim}(W)} \cdot \int_{\exp _{A^{2}}^{-1}(W) \cap\left(\mathcal{D} \times B_{r}\right)}\left(\pi d d^{c} H^{2}(z, z)\right)^{\wedge \operatorname{dim}(W)} .
\end{aligned}
$$

En effet, par hypothèse sur $r$, la fonction $\exp _{A}$ est injective sur $B_{r}$, et par suite le changement de domaine d'intégration est licite et la première ligne s'obtient en tenant compte de la définition des plongements étirés $\Phi_{\ell}^{2}$. Comme

$$
\left(\pi d d^{c} H^{2}(z, z)\right)^{\wedge \operatorname{dim}(W)}=\left(\pi d d^{c} H\left(z_{1}, z_{1}\right)+\pi d d^{c} H\left(z_{2}, z_{2}\right)\right)^{\wedge \operatorname{dim}(W)}
$$

se développe en somme de formes positives, on peut encore écrire

$$
\mathcal{V} \geqslant\left(4^{\ell}+1\right)^{\operatorname{dim}(W)} \cdot \int_{\exp _{A}^{-1}\left[W \cap \pi_{2}^{-1}(0)\right]} \mathcal{V}\left(z_{1}\right) \cdot\left(\pi d d^{c} H\left(z_{1}, z_{1}\right)\right)^{\wedge \operatorname{dim}\left(W \cap \pi_{2}^{-1}(0)\right)}
$$

avec

$$
\mathcal{V}\left(z_{1}\right):=\int_{\exp _{A^{2}}^{-1}(W) \cap\left(\left\{z_{1}\right\} \times B_{r}\right)}\left(\pi d d^{c} H\left(z_{2}, z_{2}\right)\right)^{\wedge\left(\operatorname{dim}(W)-\operatorname{dim}\left(W \cap \pi_{2}^{-1}(0)\right)\right)},
$$

et en identifiant $W \cap \pi_{2}^{-1}(0)$ à sa projection sur le premier facteur de $A^{2}$. Finalement, comme le nombre $\int_{\exp _{A}^{-1}\left[W \cap \pi_{2}^{-1}(0)\right]}\left(\pi d d^{c} H\left(z_{1}, z_{1}\right)\right)^{\wedge \operatorname{dim}\left(W \cap \pi_{2}^{-1}(0)\right)}$ est égal à (toujours par la proposition 4.1)

$$
\int_{A} \int_{W \cap \pi_{2}^{-1}(0)}\left(\Theta \circ \tau_{x_{1}}\right)^{\star}\left(\Omega_{\mathbb{P}_{N}}\right)^{\wedge \operatorname{dim}\left(W \cap \pi_{2}^{-1}(0)\right)} \wedge d \nu_{A}\left(x_{1}\right)=\operatorname{deg}\left(W \cap \pi_{2}^{-1}(0)\right),
$$

il suffit de montrer $\mathcal{V}\left(z_{1}\right) \geqslant r^{2\left(\operatorname{dim}(W)-\operatorname{dim}\left(W \cap \pi_{2}^{-1}(0)\right)\right)}$. Mais ceci résulte, après changement de variable ramenant $\pi H$ à sa forme orthonormale, de la croissance de la masse de Lelong (voir [Gr-Ha], pages 390-391 ou [Ph]-I, fait 1). 
Nous généralisons ensuite le théorème 3.1 de [Wa] en y considérant des fonctions de $\mathbb{C}^{b}$ à valeurs dans $\mathbb{C}^{n}$, où $b$ et $n$ sont des entiers $\geq 1$, analytiques sur une boule de la forme $\left\{\boldsymbol{z} \in \mathbb{C}^{b} ;\|\boldsymbol{z}\| \leq R\right\} \subset \mathbb{C}^{b}$. Si $F$ est une telle fonction et $F_{1}, \ldots, F_{n}$ ses composantes on notera

$$
|F|_{R}:=\max _{i=1, \ldots, n}\left|F_{i}\right|_{R}=\max _{i=1, \ldots, n} \sup _{\|\boldsymbol{z}\| \leq R}\left|F_{i}(\boldsymbol{z})\right| .
$$

Cette généralisation du résultat de $M$. WALDSCHMIDT (ce dernier se restreignait au cas $n=1$ ) permet de disposer de plusieurs fonctions analytiques simultanément petites, et par suite, en faisant opérer le groupe de GALOIs, de travailler avec toutes les places archimédiennes simultanément. C'est cette approche qui nous permet d'éliminer la dépendance en le corps de définition de $A$ dans le théorème 1.1 .

Proposition 4.3. Soient $L, b$ et $n$ des entiers strictement positifs, $S, U, R$ et $E$ des nombres réels positifs et $f_{1}, \ldots, f_{L}$ des fonctions continues sur la boule $\left\{\boldsymbol{z} \in \mathbb{C}^{b} ;\|\boldsymbol{z}\| \leq R\right\}$ de $\mathbb{C}^{b}$ et analytiques à l'intérieur de cette boule, à valeurs dans $\mathbb{C}^{n}$. On suppose ${ }^{10}$ que $5 \leq S \leq U, \max (\mathrm{e} ; \sqrt{b}) \leq E \leq \mathrm{e}^{U}$, ainsi que

$$
\left\{\begin{array}{c}
\sum_{\lambda=1}^{L}\left|f_{\lambda}\right|_{R} \leq \mathrm{e}^{U}, \\
8 n U(4 U+b \log (E))^{b} \leq(b-1) ! L S(\log (E))^{b} .
\end{array}\right.
$$

Alors, il existe des entiers $p_{1}, \ldots, p_{L}$ non tous nuls, de valeurs absolues $\leq \mathrm{e}^{S}$ tels que

$$
\left|\sum_{\lambda=1}^{L} p_{\lambda} f_{\lambda}\right|_{r} \leq \mathrm{e}^{-U}
$$

où $r:=R / E$.

Démonstration. Soit $T \in[4,4 U]$ le plus petit entier $\geq 4 U / \log (E)$, considérons le système d'inéquations d'inconnues $p_{\lambda}$ donné par

$$
\left|\sum_{\lambda=1}^{L} p_{\lambda} f_{\lambda, i, \boldsymbol{\tau}}\right| \cdot r^{|\boldsymbol{\tau}|} \leq \frac{1}{2} T^{-b} \mathrm{e}^{-U}
$$

lorsque $i=1, \ldots, n, \boldsymbol{\tau}=\left(\tau_{1}, \ldots, \tau_{b}\right) \in \mathbb{N}^{b}$, où $|\boldsymbol{\tau}|:=\tau_{1}+\cdots+\tau_{b}<T$ et $f_{\lambda, i, \boldsymbol{\tau}}$ désigne le $\boldsymbol{\tau}$-ième coefficient de TAYLOR de la $i$-ième composante de $f_{\lambda}$. Le nombre d'inconnues est $L$ et, par définition de $T$, le nombre d'inéquations est :

$$
n\left(\begin{array}{c}
T+b-1 \\
b
\end{array}\right) \leq \frac{n}{b !}\left(\frac{4 U+b \log (E)}{\log (E)}\right)^{b} .
$$

$\overline{10}$ On notera qu'il convient également de renforcer comme nous le faisons ici l'hypothèse $E \geq \mathrm{e}$ de [Wa]. Ceci permet de corriger les estimations liées aux inégalités de CAUCHY de loc. cit. 
On a

$$
\sum_{\lambda=1}^{L}\left|f_{\lambda, i, \boldsymbol{\tau}}\right| \cdot r^{|\boldsymbol{\tau}|} \leq \sum_{\lambda=1}^{L}\left|f_{\lambda}\right|_{r \sqrt{b}} \leq \mathrm{e}^{U}
$$

et on vérifie que l'on se trouve dans les conditions d'application du lemme 3.3 (principe des tiroirs) de [Wa] car nos hypothèses entraînent que

$$
\left(3 U+b \log (T)+\frac{5 \log (2)}{2}\right) \cdot \frac{2 n}{b !} \cdot\left(\frac{4 U+b \log (E)}{\log (E)}\right)^{b} \leq \frac{8 n U}{(b-1) !}\left(\frac{4 U+b \log (E)}{\log (E)}\right)^{b}
$$

est majoré par $L S$.

On obtient donc l'existence d'entiers $p_{1}, \ldots, p_{L}$ non tous nuls, de valeurs absolues $\leq \mathrm{e}^{S}$ solutions du système (14) et donc tels que

$$
\sum_{|\boldsymbol{\tau}|<T}\left|\sum_{\lambda=1}^{L} p_{\lambda} f_{\lambda, i, \boldsymbol{\tau}}\right| \cdot r^{|\boldsymbol{\tau}|} \leq \frac{\mathrm{e}^{-U}}{2}
$$

pour tous $i=1, \ldots, n$. Le lemme 3.4 de [Wa] (lemme de ScHWARZ approché) entraîne pour toutes les composantes de la fonction $F=\sum_{\lambda=1}^{L} p_{\lambda} f_{\lambda}$ :

$$
\begin{aligned}
\left|F_{i}\right|_{r} & \leq(\sqrt{T}+1) E^{-T}\left|F_{i}\right|_{R}+\sum_{|\boldsymbol{\tau}|<T}\left|F_{i, \boldsymbol{\tau}}\right| \cdot r^{|\boldsymbol{\tau}|} \\
& \leq \frac{1}{2} E^{-T} \mathrm{e}^{3 U}+\frac{1}{2} \mathrm{e}^{-U} \\
& \leq \mathrm{e}^{-U}
\end{aligned}
$$

car

$$
\left|F_{i}\right|_{R} \leq \mathrm{e}^{S} \cdot \sum_{\lambda=1}^{L}\left|f_{\lambda, i}\right|_{R} \leq \mathrm{e}^{2 U}
$$

et la conclusion s'en déduit. La proposition 4.3 est donc entièrement établie.

\subsection{Hauteurs et intersections}

On reprend les notations du paragraphe précédent et en particulier les plongements $\Theta, \Phi_{\ell}$ de $A$ dans $\mathbb{P}_{N}, \mathbb{P}_{N^{\prime}}$ respectivement et $\Theta^{2}, \Phi_{\ell}^{2}$ de $A^{2}$ dans $\mathbb{P}_{N}^{2}, \mathbb{P}_{N^{\prime}}^{2}$ respectivement, avec $N^{\prime}+1=(N+1)^{2}$ et $\ell$ un entier $\geqslant 1$.

Soit $W \subset A^{2}$, on suppose que $\pi_{2}(W)=B$ est une sous-variété abelienne de $A$ où $\pi_{2}$ désigne la projection sur le second facteur de $A^{2}$. On note $\boldsymbol{k}^{\prime}$ un corps de définition de $A$ et $B$; à toute place à l'infini $v$ de $\boldsymbol{k}^{\prime}$ on associe un plongement $\sigma_{v}$ de $\boldsymbol{k}^{\prime}$ dans $\mathbb{C}$ et on note $\theta_{v, 0}, \ldots, \theta_{v, N}$ les fonctions thêta paramétrant $\sigma_{v}(A)(\mathbb{C})$. Suivant J.-B. Bost [Bo], §. 2, on introduit la notion suivante : 
Définition 4.4. On appelle rayon d'injectivité de $\sigma_{v}(A)(\mathbb{C})$, et on note $\mathcal{R}_{\text {inj, }, v}$, la plus petite norme de RIEMANn d'une période non nulle de $\sigma_{v}(A)(\mathbb{C})$ :

$$
\mathcal{R}_{\mathrm{inj}, v}(A):=\min \left\{\pi \cdot H_{v}(\lambda, \lambda) ; \lambda \in \Lambda_{v} \backslash\{0\}\right\}^{1 / 2} .
$$

Et, si pour chaque place archimédienne $v$ de $\boldsymbol{k}^{\prime}$, on a identifié $\sigma_{v}(A)(\mathbb{C})$ à un tore complexe $\mathbb{C}^{g} /\left(\mathbb{Z}^{g}+\tau_{v} \mathbb{Z}^{g}\right)$, avec $\tau_{v}$ dans le « domaine fondamental » de l'espace de Siegel pour l'action de $\mathrm{Sp}_{2 g}(\mathbb{Z})$ (voir appendice, §. 6), on appelle rayon d'injectivité absolu de $A$, que l'on note $\mathcal{R}_{\text {inj }}$, la quantité

$$
\mathcal{R}_{\text {inj }}:=\min \left\{\mathcal{R}_{\text {inj }, v} ; v \mid \infty\right\} .
$$

On pose,

$$
\mathcal{V}_{\text {max }}:=8 \operatorname{dim}(B) \cdot\left(\max \{\mathrm{e} ; \sqrt{\operatorname{dim}(B)}\} \mathcal{R}_{\text {inj }}\right)^{2 \operatorname{dim}(B)}
$$

et, pour $z_{0} \in \sigma_{v}\left(T_{A(\mathbb{C})}\right)$,

$$
B_{r, v}\left(z_{0}\right):=\left\{z \in T_{\sigma_{v}(A)(\mathbb{C})} ; \pi \cdot H_{v}\left(z-z_{0}, z-z_{0}\right) \leqslant r^{2}\right\} .
$$

On note $\tilde{\theta}_{v}=\left(\tilde{\theta}_{v, 0}, \ldots, \tilde{\theta}_{v, 0}\right)$ les fonctions analytiques sur $\mathbb{C}^{g}$ déduites des $\theta_{v, i}$ après changement de variables ramenant $\pi H_{v}$ sur sa forme orthonormale et $T_{\sigma_{v}(B)(\mathbb{C})}$ sur un sous-espace de $\operatorname{dimension} \operatorname{dim}(B)$ fixé de $\mathbb{C}^{g}$, que nous noterons $T_{B(\mathbb{C})}$. Il existe un réel $c_{2}>0$ tel que pour toute place archimédienne $v$ de $\boldsymbol{k}^{\prime}$ :

$$
\exp \left(-c_{2}\right) \leq \sup _{0 \leq i \leq N}\left|\tilde{\theta}_{v, i}(z)\right| \leq \exp \left(\|z\|^{2}+c_{2}\right) .
$$

Cela résulte en effet, après le changement de variables opéré, de [Da], théorème 3.1.

D'après la proposition 3.7 les produits $\tilde{\theta}_{v, i}\left(z+z_{0}\right) \tilde{\theta}_{v, j}\left(z-z_{0}\right)$ s'expriment comme formes de bidegré $(2,2)$ en les $\tilde{\theta}_{v, i}(z)$ et $\tilde{\theta}_{v, i}\left(z_{0}\right)$. On a de plus

$$
\mathrm{e}^{-c_{v}} \leq \frac{\left\|\tilde{\theta}_{v}\left(z+z_{0}\right)\right\|_{v} \cdot\left\|\tilde{\theta}_{v}\left(z-z_{0}\right)\right\|_{v}}{\left\|\tilde{\theta}_{v}(z)\right\|_{v}^{2} \cdot\left\|\tilde{\theta}_{v}\left(z_{0}\right)\right\|_{v}^{2}} \leq \mathrm{e}^{c_{v}}
$$

avec $\sum_{v} \frac{\left[K_{v}: \mathbf{Q}_{v}\right]}{[K: \mathbf{Q}]} \cdot c_{v} \leq c_{3}:=2\left(4^{g}-1\right) h(A)+2 g \log (2)$.

Dans la situation ci-dessus, on dispose du :

Lemme 4.5. Soient $\xi \in A^{2}(\overline{\mathbb{Q}})$ un point de torsion, $0<\mathcal{V} \leq \operatorname{deg}(B)$ un réel et $r:=\frac{(\mathcal{V} / 8 \operatorname{dim}(B))^{1 / 2 \operatorname{dim}(B)}}{3 \max (e ; \sqrt{\operatorname{dim}(B)})}$, pour tout entier $\ell \geq \log _{2}\left(\frac{16\left(c_{2}+c_{3}+3 c_{0}(\Theta)+\log (N+1)\right)}{(\mathcal{V} / 8 \operatorname{dim}(B))^{1 / \operatorname{dim}(B)}}\right)$, on $a$ :

$$
\frac{\hat{h}(W)}{\operatorname{deg}(W)} \geqslant \frac{1}{8} \cdot\left(\frac{\mathcal{V}_{\xi}}{\operatorname{deg}(W)}-\frac{\mathcal{V}}{\operatorname{deg}(B)}-\frac{1}{2^{\ell}}\right) \cdot\left(\frac{\mathcal{V}}{8 \operatorname{dim}(B)}\right)^{\frac{1}{\operatorname{dim}(B)}}
$$

avec

$$
\mathcal{V}_{\xi}:=\left(4^{\ell}+1\right)^{-\operatorname{dim}(W)} \cdot \sum_{v \mid \infty} \frac{\left[\boldsymbol{k}_{v}^{\prime}: \mathbb{Q}_{v}\right]}{\left[\boldsymbol{k}^{\prime}: \mathbb{Q}\right]} \cdot \int_{\sigma_{v}(W) \cap \pi_{2}^{-1}\left(\exp _{\sigma_{v}(A)}\left(B_{r, v}(0)\right)\right)}\left(\Phi_{\ell}^{2} \circ \tau_{\xi}\right)^{\star}\left(\Omega_{\mathbb{P}_{N^{\prime}}^{2}}^{\wedge \operatorname{dim}(W)}\right)
$$


Démonstration. Dans toute la démonstration les $o(\cdot)$ sont relatifs à $\delta$ tendant vers l'infini. Pour $\delta$ assez grand, le nombre de monômes de degré $\delta$ linéairement indépendants sur $\overline{\mathbb{Q}}$ modulo l'idéal de définition de $\Phi_{\ell}(B)$ est

$$
\mathcal{H}\left(\Phi_{\ell}(B) ; \delta\right)=\frac{\operatorname{deg}\left(\Phi_{\ell}(B)\right) \cdot \delta^{\operatorname{dim}(B)}}{\operatorname{dim}(B) !}(1+o(1)) .
$$

En multipliant chacun de ces monômes par les éléments $\xi_{1}, \ldots, \xi_{\left[\boldsymbol{k}^{\prime}: \mathbb{Q}\right]}$ d'une base entière de $\boldsymbol{k}^{\prime}$ sur $\mathbb{Q}$, on en déduit $\left[\boldsymbol{k}^{\prime}: \mathbb{Q}\right] \cdot \frac{\operatorname{deg}\left(\Phi_{\ell}(B)\right) \cdot \delta^{\operatorname{dim}(B)}}{\operatorname{dim}(B) !}(1+o(1))$ monômes linéairement indépendants sur $\mathbb{Q}$.

Pour $\boldsymbol{\lambda} \in \mathbb{N}^{N^{\prime}+1},|\boldsymbol{\lambda}|=\delta$, et $m=1, \ldots,\left[\boldsymbol{k}^{\prime}: \mathbb{Q}\right]$, notons $f_{\boldsymbol{\lambda}, m}$ le vecteur dont les composantes $f_{\boldsymbol{\lambda}, m, v}(z)$, pour chaque place archimédienne $v$ de $\boldsymbol{k}^{\prime}$, sont les monômes

$$
f_{\boldsymbol{\lambda}, m, v}(z):=\sigma_{v}\left(\xi_{m}\right) \cdot \prod_{0 \leq i, j \leq N}\left(\tilde{\theta}_{v, i}(z) \tilde{\theta}_{v, j}\left(2^{\ell} z\right)\right)^{\lambda_{i, j}}
$$

Les fonctions $\left.f_{\boldsymbol{\lambda}, m}\right|_{T_{B(\mathbb{C})}}$ sont des fonctions analytiques de $\operatorname{dim}(B)$ variables à valeurs dans $\mathbb{C}^{\left[\boldsymbol{k}^{\prime}: \mathbb{Q}\right]}$. Pour toute place archimédienne $v$ de $\boldsymbol{k}^{\prime}$ on déduit de (15) les inégalités suivantes :

$$
\begin{aligned}
& \sum_{m=1}^{\left[\boldsymbol{k}^{\prime}: \mathbb{Q}\right]} \sum_{|\boldsymbol{\lambda}|=\delta}\left|f_{\boldsymbol{\lambda}, m, v}(z)\right| \geqslant \exp \left(-\delta\left(2 c_{2}+o(1)\right)\right) \\
& \sum_{m=1}^{\left[\boldsymbol{k}^{\prime}: \mathbb{Q}\right]} \sum_{|\boldsymbol{\lambda}|=\delta}\left|f_{\boldsymbol{\lambda}, m, v}(z)\right| \leqslant \exp \left(\delta\left(4^{\ell}+1\right) \cdot\|z\|^{2}+2 \delta\left(c_{2}+\log (N+1)+o(1)\right)\right)
\end{aligned}
$$

car $\log \max \left\{\left|\xi_{1}\right|_{v},\left|\xi_{1}\right|_{v}^{-1}, \ldots,\left|\xi_{\left[\boldsymbol{k}^{\prime}: \mathbb{Q}\right]}\right|_{v},\left|\xi_{\left[\boldsymbol{k}^{\prime}: \mathbb{Q}\right]}\right|_{v}^{-1}\right\}=o(\delta)$.

On applique la proposition 4.3 avec

$b=\operatorname{dim}(B), \quad L=\left[\boldsymbol{k}^{\prime}: \mathbb{Q}\right] \cdot \mathcal{H}\left(\Phi_{\ell}(B) ; \delta\right), \quad R=r \cdot \max \{\mathrm{e} ; \sqrt{b}\}, \quad S=\frac{U \cdot \mathcal{V}}{\operatorname{deg}(B)} \leqslant U$

et une famille $\mathcal{L}$ de fonctions $\left.f_{\boldsymbol{\lambda}, m}\right|_{T_{B(\mathbb{C})}}$ de $b$ variables complexes, correspondant à des monômes indépendants sur $\mathbb{Q}$ modulo l'idéal de définition de $\Phi_{\ell}(B)$. On pose enfin

$$
U=\frac{1}{8} \cdot\left(\frac{(b-1) ! L \mathcal{V}}{8\left[\boldsymbol{k}^{\prime}: \mathbb{Q}\right] \operatorname{deg}(B)}\right)^{\frac{1}{b}} \cdot \max (1 ; \log (b) / 2) .
$$

Comme $\ell \geq \frac{1}{2} \log _{2}\left(\frac{2^{8}\left(c_{2}+\log (N+1)\right)}{(\mathcal{V} / 8 b)^{1 / b}}\right)$ par hypothèse, en supposant $\delta$ assez grand on a (vérification numérique immédiate pour la première inégalité; on remplace $r$ par sa valeur pour la deuxième) :

$$
U \geqslant \frac{\delta \cdot\left(4^{\ell}+1\right)}{8+o(1)} \cdot\left(\frac{\mathcal{V}}{8 b}\right)^{\frac{1}{b}} \geqslant \delta\left(4^{\ell}+1\right) r^{2} \cdot \max \left(\mathrm{e}^{2} ; b\right)+\delta\left(2 c_{2}+\log (N+1)+o(1)\right) .
$$


Par ailleurs, on vérifie numériquement que :

$$
8\left[\boldsymbol{k}^{\prime}: \mathbb{Q}\right] U(4 U+b \log \max \{\mathrm{e} ; \sqrt{b}\})^{b} \leq(b-1) ! L S(\log \max \{\mathrm{e} ; \sqrt{b}\})^{b} .
$$

On est donc dans les conditions d'application de la proposition 4.3 et on obtient l'existence d'entiers $p_{\boldsymbol{\lambda}, m}$ de modules $\leqslant \mathrm{e}^{S}$ tels que la fonction

$$
F:=\left.\sum_{(\boldsymbol{\lambda}, m) \in \mathcal{L}} p_{\boldsymbol{\lambda}, m} \cdot f_{\boldsymbol{\lambda}, m}\right|_{T_{B(\mathbb{C})}}
$$

satisfasse

$$
0<|F|_{r}=\sup _{v,\|z\| \leqslant r}\left|F_{v}(z)\right| \leqslant \mathrm{e}^{-U}
$$

On note $q$ la forme de $\boldsymbol{k}^{\prime}\left[X_{0}, \ldots, X_{N^{\prime}}\right]$ dont les coefficients sont les combinaisons linéaires des $p_{\boldsymbol{\lambda}, m}$ correspondant aux multiples d'un même monôme unitaire par les éléments de la base de $\boldsymbol{k}^{\prime}$ sur $\mathbb{Q}$ fixée $\left(i . e \cdot \sum_{m=1}^{\left[\boldsymbol{k}^{\prime}: \mathbb{Q}\right]} p_{\boldsymbol{\lambda}, m} \xi_{m}\right)$. On a ainsi $F_{v}(z)=$ $\sigma_{v}(q)\left(\tilde{\Phi}_{v}(z)\right)$ pour toute place archimédienne $v$ de $\boldsymbol{k}^{\prime}$, où

$$
\begin{aligned}
\tilde{\Phi}_{v}: \mathbb{C}^{g} & \mathbb{P}_{N^{\prime}}(\mathbb{C}) \\
z & \longmapsto\left(\cdots: \tilde{\theta}_{v, i}(z) \tilde{\theta}_{v, j}\left(2^{\ell} z\right): \cdots\right)_{\substack{0 \leq i \leq N \\
0 \leq j \leq N}} .
\end{aligned}
$$

Et donc, pour toute place archimédienne $v$ de $\boldsymbol{k}^{\prime}$ et tout $x=\tilde{\Phi}_{v}(z)$ avec $\|z\| \leqslant r$, on a encore, en tenant compte de la première inégalité de la relation (17),

$$
\begin{aligned}
\frac{\left|\sigma_{v}(q)(x)\right|_{v}}{\|x\|_{v}^{\delta}} & =\frac{\left|F_{v}(z)\right|_{v}}{\left\|\tilde{\Phi}_{v}(z)\right\|_{v}^{\delta}} \\
& \leqslant|F|_{r} \cdot \exp \left(\left(2 c_{2}+o(1)\right) \delta\right) \\
& \leqslant \exp \left(-U+\left(2 c_{2}+o(1)\right) \delta\right),
\end{aligned}
$$

tandis que pour tout $x \in \sigma_{v}(B)(\mathbb{C})$ on a

$$
\frac{\left|\sigma_{v}(q)(x)\right|_{v}}{\|x\|_{v}^{\delta}} \leqslant \mathrm{e}^{S+c_{4}}(N+1)^{2 \delta} .
$$

Pour les places ultramétriques $v$, comme $q$ est à coefficients entiers algébriques, on a pour tout $x \in B\left(\mathbb{C}_{v}\right)$

$$
\frac{\left|\sigma_{v}(q)(x)\right|_{v}}{\|x\|_{v}^{\delta}} \leqslant 1 .
$$

Sans perte de généralité on peut supposer que la variété abelienne $B$ n'est pas contenue dans le diviseur découpé par la forme linéaire $X_{0}$. Considérons la forme $X_{0}^{\delta} q(\boldsymbol{Y})$, que l'on peut voir dans le plongement de SEGRE de $\Phi_{\ell}^{2}\left(A^{2}\right)$. On sait (voir proposition 3.7) que l'image inverse par le morphisme $\xi$, introduit à la formule $(2)$, de $X_{0}^{\delta} q(\boldsymbol{Y})$ s'écrit comme une forme bihomogène de bidegré $(2 \delta, 2 \delta)$ sur $\Phi_{\ell}^{2}\left(A^{2}\right)$. En spécialisant les variables $\boldsymbol{Y}$ en des coordonnées du point de torsion 
$\pi_{2}(\xi)$ fixé dans l'énoncé, on obtient une forme $Q$ de degré $2 \delta$ sur $\Phi_{\ell}(A)$, qui satisfait pour toute place $v$ et $x \in \Phi_{\ell}(B)\left(\mathbb{C}_{v}\right)$

$$
\frac{\left|\sigma_{v}(Q)(x)\right|_{v}}{\|x\|_{v}^{2 \delta} \cdot\left\|\pi_{2}(\xi)\right\|_{v}^{2 \delta}}=\frac{\left|\sigma_{v}(q)\left(x-\pi_{2}(\xi)\right)\right|_{v} \cdot\left|\sigma_{v}\left(X_{0}\right)\left(x+\pi_{2}(\xi)\right)\right|_{v}^{\delta}}{\|x\|_{v}^{2 \delta} \cdot\left\|\pi_{2}(\xi)\right\|_{v}^{2 \delta}} .
$$

Intersectons $\Phi_{\ell}^{2} \circ \tau_{\xi}(W)$ avec $\pi_{2}^{\star}(Q)$, la hauteur relative $h_{\Phi_{\ell}^{2} \circ \tau_{\xi}(W)}\left(\pi_{2}^{\star}(Q)\right)$ intervenant dans le théorème de BÉzout arithmétique (cf. [Ph]-III, prop. 4) s'écrit

$$
\frac{1}{\operatorname{deg}\left(\Phi_{\ell}^{2} \circ \tau_{\xi}(W)\right)} \cdot \sum_{v} \frac{\left[\boldsymbol{k}_{v}^{\prime}: \mathbb{Q}_{v}\right]}{\left[\boldsymbol{k}^{\prime}: \mathbb{Q}\right]} \cdot \int_{\sigma_{v} \circ \Phi_{\ell}^{2} \circ \tau_{\xi}(W)} \log \left(\frac{\left|\sigma_{v}(Q)\left(\pi_{2}(y)\right)\right|_{v}}{\|y\|_{v}^{2 \delta}}\right) \cdot \Omega_{\Phi_{\ell}^{2} \circ \tau_{\xi}(W), v}(y) .
$$

Notons $I_{v}$ l'intégrale ci-dessus divisée par $\operatorname{deg}\left(\Phi_{\ell}^{2} \circ \tau_{\xi}(W)\right)$, on vérifie que $\operatorname{deg}\left(\Phi_{\ell}^{2} \circ \tau_{\xi}(W)\right) . I_{v}$ est majoré par

$$
\int_{\sigma_{v} \circ \Phi_{\ell}^{2} \circ \tau_{\xi}(W)} \log \left(\frac{\left|\sigma_{v}(Q)\left(\pi_{2}(y)\right)\right|_{v}}{\left\|\pi_{2}(y)\right\|_{v}^{2 \delta} \cdot\left\|\pi_{2}(\xi)\right\|_{v}^{2 \delta}}\right) \cdot \Omega_{\Phi_{\ell}^{2} \circ \tau_{\xi}(W), v}(y)+2 \delta \log \left(\left\|\pi_{2}(\xi)\right\|_{v}\right)
$$

car $\|y\|_{v} \geq\left\|\pi_{2}(y)\right\|_{v}$, puis, en tenant compte de la formule (16),

$$
\begin{array}{r}
\int_{\sigma_{v} \circ \Phi_{\ell}^{2} \circ \tau_{\xi}(W)} \log \left(\frac{\left|\sigma_{v}(q)\left(\pi_{2}(y-\xi)\right)\right|_{v} \cdot\left|\sigma_{v}\left(X_{0}\right)\left(\pi_{2}(y+\xi)\right)\right|_{v}^{\delta}}{\left.\| \pi_{2}(y-\xi)\right)\left\|_{v}^{\delta} \cdot\right\| \pi_{2}(y+\xi) \|_{v}^{\delta}}\right) \cdot \Omega_{\Phi_{\ell}^{2} \circ \tau_{\xi}(W), v}(y) \\
+\delta\left(c_{v}+2 \log \left(\left\|\pi_{2}(\xi)\right\|_{v}\right)\right) .
\end{array}
$$

La formule (20) nous assure alors que, pour les places ultramétriques,

$$
\operatorname{deg}\left(\Phi_{\ell}^{2} \circ \tau_{\xi}(W)\right) \cdot I_{v} \leq \delta\left(c_{v}+2 \log \left(\left\|\pi_{2}(\xi)\right\|_{v}\right)\right) .
$$

Tandis que, pour les places archimédiennes, on vérifie que $\operatorname{deg}\left(\Phi_{\ell}^{2} \circ \tau_{\xi}(W)\right) . I_{v}$ est majoré par

$$
\begin{aligned}
\int_{\sigma_{v} \circ \Phi_{\ell}^{2} \circ \tau_{\xi}(W) \cap \pi_{2}^{-1}\left(\exp _{\sigma_{v}(A)}\left(B_{r, v}\left(z_{v}\right)\right)\right)} \log \left(\frac{\left|\sigma_{v}(q)\left(\pi_{2}(y-\xi)\right)\right|_{v}}{\left\|\pi_{2}(y-\xi)\right\|_{v}^{\delta}}\right) \cdot \Omega_{\Phi_{\ell}^{2} \circ \tau_{\xi}(W), v}(y) \\
+S+\delta\left(c_{v}+2 \log \left((N+1)\left\|\pi_{2}(\xi)\right\|_{v}\right)\right)+c_{4}
\end{aligned}
$$

où $\exp _{\sigma_{v}(A)}\left(z_{v}\right)=\sigma_{v} \circ \pi_{2}(\xi)$. En effet, on majore pour ces places archimédiennes l'intégrant par $S+2 \delta \log (N+1)+c_{4}$ sur le complémentaire du domaine d'intégration mis à part, grâce à la formule (19), puis par

$$
\log \left(\frac{\left|\sigma_{v}(q)\left(\pi_{2}(y-\xi)\right)\right|_{v}}{\left\|\pi_{2}(y-\xi)\right\|_{v}^{\delta}}\right)
$$

sur ce domaine. Enfin, $\frac{\mid \sigma_{v}(q)\left(\left.\pi_{2}(y-\xi)\right|_{v}\right.}{\left\|\pi_{2}(y-\xi)\right\|_{v}^{\delta}}$ est majoré par $\mathrm{e}^{-U+\left(2 c_{2}+o(1)\right) \delta}$ sur la boule $\exp _{\sigma_{v}(A)}\left(B_{r, v}\left(z_{v}\right)\right)$, d'après (18).

D'où, en sommant sur toutes les places et puisque la somme sur toutes les places archimédiennes du volume du dernier domaine d'intégration considéré est 
$\left(4^{\ell}+1\right)^{\operatorname{dim}(W)} \cdot \mathcal{V}_{\xi}=\frac{\operatorname{deg}\left(\Phi_{\ell}^{2} \circ \tau_{\xi}(W)\right)}{\operatorname{deg}(W)} \cdot \mathcal{V}_{\xi}$, par définition de $\mathcal{V}_{\xi}$,

$h_{\Phi_{\ell}^{2} \circ \tau_{\xi}(W)}\left(\pi_{2}^{\star}(Q)\right) \leq S+\delta\left(c_{3}+2 h\left(\pi_{2}(\xi)\right)+2 \log (N+1)\right)+c_{4}-\frac{\left(U-\left(2 c_{2}+o(1)\right) \delta\right) \cdot \mathcal{V}_{\xi}}{\operatorname{deg}(W)}$.

On applique donc le théorème de BÉzout arithmétique à l'intersection de $\Phi_{\ell}^{2} \circ \tau_{\xi}(W)$ et de $\tau_{\xi}(\mathcal{Z}(Q))$. La hauteur de l'intersection étant toujours $\geqslant 0$ et le degré et la hauteur normalisée étant invariants par translations par les points de torsion (voir [Ph]-I, prop. 9), on obtient

$$
\begin{aligned}
& \frac{\hat{h}(W)}{\operatorname{deg}(W)}=\frac{\hat{h}\left(\Phi_{\ell}^{2} \circ \tau_{\xi}(W)\right)}{\left(4^{\ell}+1\right) \operatorname{deg}\left(\Phi_{\ell}^{2} \circ \tau_{\xi}(W)\right)} \\
\geqslant & \frac{h\left(\Phi_{\ell}^{2} \circ \tau_{\xi}(W)\right)}{\left(4^{\ell}+1\right) \operatorname{deg}\left(\Phi_{\ell}^{2} \circ \tau_{\xi}(W)\right)}-\frac{2 c_{0}(\Theta)}{4^{\ell}+1} \\
\geqslant & -\frac{h_{\Phi_{\ell}^{2} \circ \tau_{\xi}(W)}\left(\pi_{2}^{\star}(Q)\right)}{\left(4^{\ell}+1\right) \delta}-\frac{2 c_{0}(\Theta)}{4^{\ell}+1} \\
\geqslant & \frac{\left(U-\left(2 c_{2}+o(1)\right) \delta\right) \cdot \mathcal{V}_{\xi}}{\left(4^{\ell}+1\right) \delta \cdot \operatorname{deg}(W)}-\frac{S+\delta\left(c_{3}+2 h\left(\pi_{2}(\xi)\right)+2 \log (N+1)\right)+c_{4}}{\left(4^{\ell}+1\right) \delta}-\frac{2 c_{0}(\Theta)}{4^{\ell}+1} \\
\geqslant & \frac{U}{\left(4^{\ell}+1\right) \delta} \cdot\left(\frac{\mathcal{V}_{\xi}}{\operatorname{deg}(W)}-\frac{\mathcal{V}}{\operatorname{deg}(B)}\right)-\frac{2 c_{2}+6 c_{0}(\Theta)+c_{3}+2 \log (N+1)+o(1)}{4^{\ell}+1}
\end{aligned}
$$

la dernière inégalité se vérifie en remplaçant $S$ par sa valeur $S=\frac{U \mathcal{V}}{\operatorname{deg}(B)}$, en majorant $h\left(\pi_{2}(\xi)\right)$ par $2 c_{0}(\Theta)$ et en notant que, par définition, $\frac{\mathcal{V}_{\xi}}{\operatorname{deg}(W)} \leq 1$.

Enfin, comme $2^{\ell} \geq \frac{16\left(c_{2}+c_{3}+3 c_{0}(\Theta)+\log (N+1)\right)}{(\mathcal{V} / 8 b)^{1 / b}}$ et $\frac{U}{\delta \cdot\left(4^{\ell}+1\right)} \geqslant \frac{1}{8+o(1)} \cdot\left(\frac{\mathcal{V}}{8 b}\right)^{\frac{1}{b}}$, on obtient

$$
\frac{\hat{h}(W)}{\operatorname{deg}(W)} \geqslant \frac{1}{8}\left(\frac{\mathcal{V}_{\xi}}{\operatorname{deg}(W)}-\frac{\mathcal{V}}{\operatorname{deg}(B)}-\frac{1}{2^{\ell}}\right) \cdot\left(\frac{\mathcal{V}}{8 b}\right)^{\frac{1}{b}}
$$

en faisant tendre $\delta$ vers l'infini. Le lemme 4.5 est donc entièrement établi.

\subsection{Preuve du théorème 1.1}

La preuve de l'inégalité $\hat{\mu}^{\text {ess }}(X) \geq \frac{\hat{h}(X)}{(\operatorname{dim}(X)+1) d(X)}$ est déjà connue (voir par exemple [Zh1], theorem 1.10 ou [Da-Phi1], théorème 1.4). Commençons par établir un résultat plus précis que le théorème 1.1 lorsque $k_{X}=1$.

Théorème 4.6. On reprend les notations et hypothèses du théorème 1.1, et l'on suppose de plus que $k_{X}=1$ (i. e. $X-X$ est une sous-variété abelienne $B_{X}$ de $A$ 
de dimension $>\operatorname{dim} X)$, on $a$ :

$$
\frac{\hat{h}(X)}{\operatorname{deg}(X)} \geqslant\left(2^{12} g\right)^{-g^{2}} \cdot \min \left\{\left(\frac{\mathcal{V}_{\max }}{\operatorname{deg}(X)^{2}}\right)^{\frac{\operatorname{dim} B_{X}+1}{\operatorname{dim} B_{X}}} ; \operatorname{deg}(X)^{-\frac{\operatorname{dim} B_{X}+1}{\operatorname{dim} B_{X}-\operatorname{dim} X}}\right\}
$$

En particulier, en posant $C_{1}(A):=\left(2^{12} g\right)^{g^{2}} . \min \left\{1 ; \mathcal{R}_{\text {inj }}\right\}^{-2\left(\operatorname{dim}\left(B_{X}\right)+1\right)}$, on a

$$
\hat{h}(X) \geqslant C_{1}(A)^{-1} \cdot \operatorname{deg}(X)^{-\operatorname{dim}\left(B_{X}\right)} .
$$

Démonstration. Soit $\ell \geq \log _{2}\left(\frac{16\left(c_{2}+c_{3}+3 c_{0}(\Theta)+\log (N+1)\right)}{(\mathcal{V} / 8 b)^{1 / b}}\right)$ un entier, on pose $b:=$ $\operatorname{dim}\left(B_{X}\right)$ et on applique le lemme 4.5 à $W$ et aux points de torsion $\xi \in A^{2}$. On en déduit en faisant la moyenne sur tous les points de $m$-torsion lorsque $m$ tend vers l'infini :

$$
\begin{aligned}
& \frac{\hat{h}(X)}{\operatorname{deg}(X)} \geqslant \frac{\hat{h}(W)}{8(16)^{\operatorname{dim}(X)} \operatorname{deg}(W)} \\
& \geqslant \frac{1}{4(16)^{\operatorname{dim}(X)+1} m^{4 g}} \cdot \sum_{\xi \in A_{m}^{2}}\left(\frac{\mathcal{V}_{\xi}}{\operatorname{deg}(W)}-\frac{\mathcal{V}}{\operatorname{deg}\left(B_{X}\right)}-\frac{1}{2^{\ell}}\right)\left(\frac{\mathcal{V}}{8 b}\right)^{\frac{1}{b}} \\
& \geqslant \frac{1}{(16)^{\operatorname{dim}(X)+2}} \cdot\left(\frac{\int_{A^{2}} \mathcal{V}_{\xi} \cdot d \nu_{A^{2}}(\xi)}{\operatorname{deg}(W)}-\frac{\mathcal{V}}{\operatorname{deg}\left(B_{X}\right)}-\frac{1}{2^{\ell}}\right) \mathcal{V}^{\frac{1}{b}} \text {. }
\end{aligned}
$$

Pour $\mathcal{V} \leq \mathcal{V}_{\max }$ le lemme 4.2 entraîne

$$
\int_{A^{2}} \mathcal{V}_{\xi} \cdot d \nu_{A^{2}}(\xi) \geqslant \mathcal{V}_{0}:=\operatorname{deg}\left(W \cap \pi_{2}^{-1}(0)\right) \cdot r^{2\left(\operatorname{dim}(W)-\operatorname{dim}\left(W \cap \pi_{2}^{-1}(0)\right)\right)}
$$

avec $r:=\frac{1}{3 \max (\mathrm{e} ; \sqrt{b})} \cdot\left(\frac{\mathcal{V}}{8 b}\right)^{\frac{1}{2 b}}$; ce choix de $r$ et la condition $\mathcal{V} \leq \mathcal{V}_{\text {max }}$ permettent d'assurer que les hypothèses du lemme 4.2 sont bien satisfaites, d'où

$$
\begin{aligned}
\frac{\hat{h}(X)}{\operatorname{deg}(X)} & \geqslant \frac{1}{(16)^{\operatorname{dim}(X)+2}} \cdot\left(\frac{\mathcal{V}_{0}}{\operatorname{deg}(W)}-\frac{\mathcal{V}}{\operatorname{deg}\left(B_{X}\right)}-\frac{1}{2^{\ell}}\right) \mathcal{V}^{\frac{1}{b}} \\
& \geqslant \frac{1}{(16)^{\operatorname{dim}(X)+2}} \cdot\left(\frac{\mathcal{V}_{0}}{\operatorname{deg}(W)}-\frac{\mathcal{V}}{\operatorname{deg}\left(B_{X}\right)}\right) \mathcal{V}^{\frac{1}{b}}
\end{aligned}
$$

en faisant tendre $\ell$ vers l'infini.

Nous posons $d=\operatorname{dim}(X)$ et $B=B_{X}$; notons que par définition, $\operatorname{dim}(W)=2 d$ et, $\operatorname{dim}\left(W \cap \pi_{2}^{-1}(0)\right)=d$. Nous allons imposer de surcroît

de sorte que

$$
\mathcal{V}:=\min \left\{\mathcal{V}_{\max } ; \operatorname{deg}(B) ; \frac{\mathcal{V}_{0} \operatorname{deg}(B)}{2 \operatorname{deg}(W)}\right\}
$$

$$
\left\{\begin{array}{l}
\mathcal{V}=\mathcal{V}_{\max } \quad \text { ou } \quad \mathcal{V}=\operatorname{deg}(B) \quad \text { ou } \\
\mathcal{V}=8 b\left(\frac{\operatorname{deg}\left(W \cap \pi_{2}^{-1}(0)\right) \cdot \operatorname{deg}(B)}{16 b \cdot\left(9 \max \left(\mathrm{e}^{2} ; b\right)\right)^{d} \operatorname{deg}(W)}\right)^{\frac{b}{b-d}}
\end{array}\right.
$$


et

$$
\frac{\hat{h}(X)}{\operatorname{deg}(X)} \geq \frac{1}{16^{d+2}} \cdot \frac{\mathcal{V}^{\frac{b+1}{b}}}{\operatorname{deg}(B)} .
$$

Dans le dernier cas, en remplaçant $r$ par sa valeur, on a :

$$
\mathcal{V}=\frac{\mathcal{V}_{0} \operatorname{deg}(B)}{2 \operatorname{deg}(W)}=\frac{\operatorname{deg}\left(W \cap \pi_{2}^{-1}(0)\right) \cdot \operatorname{deg}(B)}{2 .\left(9 \max \left(\mathrm{e}^{2} ; b\right)\right)^{d} \operatorname{deg}(W)} \cdot\left(\frac{\mathcal{V}}{8 b}\right)^{\frac{d}{b}},
$$

ce qui donne bien la valeur de $\mathcal{V}$ annoncée. On a donc (toujours dans le dernier cas)

$$
\frac{\hat{h}(X)}{\operatorname{deg}(X)} \geqslant \frac{b \operatorname{deg}(B)^{\frac{d+1}{b-d}}}{2^{4 d+5}} \cdot\left(\frac{\operatorname{deg}\left(W \cap \pi_{2}^{-1}(0)\right)}{16 b\left(9 \max \left(\mathrm{e}^{2} ; b\right)\right)^{d} \operatorname{deg}(W)}\right)^{\frac{b+1}{b-d}} .
$$

D'où, puisque

$$
\frac{\operatorname{deg}\left(W \cap \pi_{2}^{-1}(0)\right)}{\operatorname{deg}(W)} \geq \frac{\operatorname{deg}(X)}{2^{4 d} \operatorname{deg}(X)^{2}} \geq \frac{1}{2^{4 d} \operatorname{deg}(X)},
$$

la minoration

$$
\frac{\hat{h}(X)}{\operatorname{deg}(X)} \geqslant \frac{b \operatorname{deg}(B)^{\frac{d+1}{b-d}}}{2^{4 d+5}} \cdot\left(\frac{1}{2^{4 d+4} b\left(9 \max \left(\mathrm{e}^{2} ; b\right)\right)^{d} \operatorname{deg}(X)}\right)^{\frac{b+1}{b-d}} .
$$

Supposons maintenant que $\mathcal{V}=\mathcal{V}_{\max }$; on a alors

$\operatorname{car} \operatorname{deg}(B) \leqslant 2^{4 d} \operatorname{deg}(X)^{2}$.

$$
\frac{\hat{h}(X)}{\operatorname{deg}(X)} \geqslant \frac{1}{2^{4 d+8}} \cdot \frac{\mathcal{V}_{\max }^{\frac{b+1}{b}}}{\operatorname{deg}(B)} \geqslant 2^{-8 d-8} \cdot\left(\frac{\mathcal{V}_{\max }}{\operatorname{deg}(X)^{2}}\right)^{\frac{b+1}{b}}
$$

Enfin, pour finir, supposons que $\mathcal{V}=\operatorname{deg}(B)$; on a :

$$
\frac{\hat{h}(X)}{\operatorname{deg}(X)} \geqslant \frac{1}{2^{4 d+8}} \cdot \operatorname{deg}(B)^{\frac{1}{b}} \geqslant 2^{-4 d-8} .
$$

Le théorème 4.6 s'en déduit en mettant ces inégalités ensemble et en majorant $b$ par $g, d$ par $g-1$, puis en remplaçant $\mathcal{V}_{\text {max }}$ par sa valeur $8 .\left(2 \max \{\mathrm{e} ; \sqrt{b}\} \mathcal{R}_{\text {inj }}\right)^{2 b}$.

Démonstration du théorème 1.1. On procède par récurrence sur $k_{X}$, le cas $k_{X}=1$ se déduisant du théorème 4.6. Posons $Y_{0}=X$ et $Y_{i}=Y_{i-1}-Y_{i-1}$ pour $i=1, \ldots, \ell$ où $\ell$ désigne le plus grand entier $<1+\frac{\log \left(k_{X}\right)}{\log (2)}$. Si $k_{X}>1$, on a $\ell \geqslant 1$ et $k_{Y_{\ell}}=1$, d'où l'on déduit par le théorème 4.6

$$
\hat{h}\left(Y_{\ell}\right) \geqslant C_{1}(A)^{-1} \operatorname{deg}\left(Y_{\ell}\right)^{-\operatorname{dim}\left(B_{Y_{\ell}}\right)},
$$

et par les considérations du paragraphe 4.1 :

$$
\hat{h}\left(Y_{i-1}\right) \geqslant 2^{-4 \operatorname{dim}\left(Y_{i-1}\right)-3} \cdot \frac{\hat{h}\left(Y_{i}\right)}{d\left(Y_{i-1}\right)} .
$$


En télescopant ces inégalités pour $i=1, \ldots, \ell$ on obtient (en notant $b$ la dimension de $\left.B_{X}\right)$

$$
\begin{aligned}
\hat{h}(X) & \geqslant 2^{-4 \sum_{i=1}^{\ell} \operatorname{dim}\left(Y_{i-1}\right)-3 \ell} \cdot \frac{\hat{h}\left(Y_{\ell}\right)}{\operatorname{deg}(X) \operatorname{deg}\left(Y_{1}\right) \ldots \operatorname{deg}\left(Y_{\ell-1}\right)} \\
& \geq \frac{2^{-4 \sum_{i=1}^{\ell} \operatorname{dim}\left(Y_{i-1}\right)-3 \ell} \cdot C_{1}(A)^{-1}}{\operatorname{deg}(X) \operatorname{deg}\left(Y_{1}\right) \ldots \operatorname{deg}\left(Y_{\ell-1}\right) \operatorname{deg}\left(Y_{\ell}\right)^{b}} .
\end{aligned}
$$

Mais, comme pour tout $i$ compris entre 1 et $\ell$,

$$
\operatorname{deg}\left(Y_{i}\right) \leqslant\left(2^{4(b-1)}\right)^{1+\cdots+2^{i-1}} \cdot \operatorname{deg}(X)^{2^{i}} \leqslant\left(2^{4(b-1)} \cdot \operatorname{deg}(X)\right)^{2^{i}}
$$

en notant que, par définition, $2^{\ell-1} \leqslant k_{X}$ et que, par construction, $B_{Y_{\ell}}=B_{X}$ est de dimension $b$, on en déduit ${ }^{11}$

$\operatorname{deg}(X) \operatorname{deg}\left(Y_{1}\right) \cdots \operatorname{deg}\left(Y_{\ell-1}\right) \operatorname{deg}\left(Y_{\ell}\right)^{b} \leq 2^{4(b-1)\left(2 k_{X}(b+1)-\ell-b-1\right)+1} \operatorname{deg}(X)^{2 k_{X}(b+1)-1}$.

Enfin, puisque $\sum_{i=1}^{\ell} \operatorname{dim}\left(Y_{i-1}\right) \leq \ell b-\frac{\ell(\ell+1)}{2}$ et $k_{X} \leq b-d$, on a

$$
2^{4 \sum_{i=1}^{\ell} \operatorname{dim}\left(Y_{i-1}\right)+3 \ell} \cdot 2^{4(b-1)\left(2 k_{X}(b+1)-1-\ell-b\right)+1} \leq 2^{8 b^{3}-12 b^{2}-8 b+16} .
$$

D'où

$$
\hat{h}(X) \geq\left(2^{8 b^{3}-12 b^{2}-8 b+16} C_{1}(A) \cdot \operatorname{deg}(X)^{2 k_{X}(b+1)-1}\right)^{-1} .
$$

Pour conclure la preuve du théorème 1.1, il suffit de remarquer (le théorème étant vide pour $g=1$, on peut supposer $g \geq b \geq 2$ )

$$
\begin{aligned}
C(A) & :=2^{8 b^{3}-12 b^{2}-8 b+16} \cdot C_{1}(A) \\
& =2^{8 b^{3}-12 b^{2}-8 b+16} \cdot\left(2^{12} b\right)^{b^{2}} \min \left\{1 ; \mathcal{R}_{\text {inj }}\right\}^{-2(b+1)} \\
& \leqslant 2^{9 g^{3}} \cdot \min \left\{1 ; \mathcal{R}_{\text {inj }}\right\}^{-2(b+1)}
\end{aligned}
$$

ce qui conduit bien au théorème 1.1 .

\section{Minorations explicites des minimums essentiels et absolus}

L'objet de ce paragraphe est de déduire du théorème 1.1 que nous venons de démontrer au paragraphe 4.3, les théorèmes 1.2 et 1.3 de l'introduction. Comme dans [Da-Phi2], l'idée sera d'utiliser une minoration effective de la fonction de HILBERT arithmétique pour contrôler le degré du « diviseur exceptionnel » contenant les petits points.

Soit donc $A$ une variété abelienne de dimension $g \geq 1$, munie d'un fibré en droites ample et symétrique $\mathcal{L}$ associé à une polarisation principale, et $\Theta$ le plongement de $A$ dans un espace projectif $\mathbb{P}_{N}$ défini au paragraphe 3.1 associé à $\mathcal{L}^{\otimes 16}$

\footnotetext{
11 Une majoration plus fine $\operatorname{de} \operatorname{deg}\left(Y_{\ell}\right)$ à cet endroit permet d'obtenir la majoration $\operatorname{de} C(A)$ en $\exp \left(c g^{2} \log (g)\right)$ mentionnée dans l'introduction.
} 
$\left(N+1=16^{g}\right)$. On suppose $A$ définie sur $\overline{\mathbb{Q}}$ et soit de plus $X$ une sous-variété algébrique de $A$ de dimension $d$, également définie sur $\overline{\mathbb{Q}}$.

On reprend le plongement étiré de $A$ :

$$
\begin{aligned}
\Phi_{\ell}: A \hookrightarrow A^{2} \hookrightarrow \mathbb{P}_{N}^{2} \underset{\mathrm{SEgRE}}{\hookrightarrow} \mathbb{P}_{N^{\prime}} \\
x \longmapsto\left(x,\left[2^{\ell}\right] x\right)
\end{aligned}
$$

avec $\ell$ un entier et $N^{\prime}+1=(N+1)^{2}$.

On sait que $X$ est définie dans $\mathbb{P}_{N}$ par des équations de degrés $\leqslant \operatorname{deg}(X)$. De plus, $\Phi_{\ell}(A)$ est définie dans $\mathbb{P}_{N^{\prime}}$ par des équations de degré $\leq 4^{\ell}+1$. On en déduit que $\Phi_{\ell}(X)$ est définie dans $\mathbb{P}_{N^{\prime}}$ par des équations de degrés $\leqslant \max \left\{4^{\ell}+1 ; \operatorname{deg}(X)\right\}$. D'après la proposition 4.2, points (i) et (iii), de [Da-Phi2], on en déduit que l'idéal de définition de $\Phi_{\ell}(X)$ dans $\overline{\mathbb{Q}}\left[Y_{0}, \ldots, Y_{N}\right]$ est sympa ${ }^{12}$ en degré $(N+1)^{2} \cdot \max \left\{4^{\ell}+\right.$ $1 ; \operatorname{deg}(X)\}$.

On obtient alors avec ces notations et cette remarque :

Lemme 5.1. Supposons que l'on ait l'inégalité :

$$
\frac{h\left(\Phi_{\ell}(X)\right)}{\operatorname{deg}\left(\Phi_{\ell}(X)\right)}>12(d+1)(4 \mathrm{e})^{d+1} \log (N+1) .
$$

Il existe alors une forme $F$ de degré $L=3(N+1)^{2} \cdot \max \left\{4^{\ell}+1 ; \operatorname{deg}(X)\right\}$, ̀̀ coefficients dans $\overline{\mathbb{Q}}$, découpant un diviseur $Z$ de $\Phi_{\ell}(X)$ telle que tout point $x \in$ $\Phi_{\ell}(X)(\overline{\mathbb{Q}})$ de hauteur au plus :

$$
(4 \mathrm{e})^{-d-1} \cdot \frac{h\left(\Phi_{\ell}(X)\right)}{\operatorname{deg}\left(\Phi_{\ell}(X)\right)}
$$

soit un élément de $Z$.

Démonstration. C'est essentiellement le corollaire 4.12 de [Da-Phi2] (minoration effective de la fonction de HILBERT arithmétique jointe à la formule du produit), où l'on a simplement tenu compte du fait que l'idéal de définition de $\Phi_{\ell}(X)$ est sympa en degré $(N+1)^{2} \cdot \max \left\{4^{\ell}+1 ; \operatorname{deg}(X)\right\}$, et ajusté la valeur numérique de $L$ afin d'assurer que les hypothèses de cet énoncé soient satisfaites.

Nous allons maintenant résumer en l'énoncé suivant les propriétés qui nous seront utiles pour la récurrrence qui va suivre, pour passer de la codimension 1 à la codimension $d$.

Proposition 5.2. Supposons que $X$ ne soit pas un translaté d'une sous-variété abelienne de $A$, notons, comme au paragraphe $4, B_{X}$ la sous-variété abelienne de

12 Rappelons que, suivant la terminologie de [Da-Phi2], un idéal de $\overline{\mathbb{Q}}\left[Y_{0}, \ldots, Y_{N}\right]$ de rang $N+1-r$ est dit sympa en degré $D$ si $I[\boldsymbol{d}]::_{\overline{\mathbb{Q}} \boldsymbol{d}]} \overline{\mathbb{Q}}\left[Y_{0}, \ldots, Y_{N}\right]_{D+d_{1}+\cdots+d_{r}-r+1} \neq(0)$, pour tout $\boldsymbol{d}=\left(d_{1}, \ldots, d_{r}\right) \in\left(\mathbb{N}^{\star}\right)^{r}$ et où l'idéal $J[\boldsymbol{d}]$ est «l'idéal engendré par $J$ augmenté des formes génériques de degré $d_{1}, \ldots, d_{r}$ dans un sur-anneau convenable »; on pourra se reporter à [Da-Phi2], définition 4.1 pour plus de précisions. 
$A$ engendrée par $X-X$ et $k_{X}$ le nombre minimal de copies de $X-X$ dont la somme vaut $B_{X}$, et reprenons les notations introduites ci-dessus. Posons de plus:

$$
\delta:=3(N+1)^{2} \max \left\{1 ; \frac{12(4 \mathrm{e})^{d+2}(d+1) \cdot c_{0}(\Theta)}{\hat{h}(X)}\right\} \cdot \operatorname{deg}(X) .
$$

Il existe alors un sous-ensemble algébrique $Z$ de $X$, défini sur $\overline{\mathbb{Q}}$, de dimension $d-1$, satisfaisant :

$$
\operatorname{deg}(Z) \leq \frac{\delta^{2}}{3(N+1)^{2}} \cdot \operatorname{deg}(X),
$$

qui contient tous les points de $X(\overline{\mathbb{Q}})$ de hauteur normalisée :

$$
\leq(4 \mathrm{e})^{-d-2} \cdot \frac{\hat{h}(X)}{\operatorname{deg}(X)} .
$$

En particulier, les points de $X(\overline{\mathbb{Q}})$ de hauteur normalisée

$$
\leq\left((4 \mathrm{e})^{d+2} C(A)\right)^{-1} \cdot \operatorname{deg}(X)^{-2 k_{X}\left(\operatorname{dim} B_{X}+1\right)}
$$

appartiennent à $Z$ et $\operatorname{deg}(Z)$ est majoré par

$$
\begin{aligned}
& \quad 3(N+1)^{2}\left(12(d+1)(4 \mathrm{e})^{d+2} c_{0}(\Theta) C(A)\right)^{2} \cdot \operatorname{deg}(X)^{4 k_{X}\left(\operatorname{dim}\left(B_{X}\right)+1\right)+1}, \\
& \text { où } C(A):=2^{9 g^{3}} \cdot \min \left\{1 ; \mathcal{R}_{\text {inj }}\right\}^{-2\left(\operatorname{dim}\left(B_{X}\right)+1\right)} .
\end{aligned}
$$

Démonstration. Commençons par choisir $\ell$ minimal, de telle sorte que :

$$
4^{\ell}+1>3(4 \mathrm{e})^{d+2} \cdot(d+1) \cdot c_{0}(\Theta) \cdot \frac{\operatorname{deg}(X)}{\hat{h}(X)} .
$$

En tenant compte de la proposition 3.14, (pour comparer la hauteur et le degré de $\Theta_{\ell}(X)$ en fonction de la hauteur normalisée et du degré de $X$ ), on a :

$$
\left(4^{\ell}+1\right) \frac{\operatorname{deg}\left(\Phi_{\ell}(X)\right)}{h\left(\Phi_{\ell}(X)\right)} \leq \frac{\operatorname{deg}(X)}{\hat{h}(X)} \frac{1}{1-\frac{2(d+1) c_{0}(\Theta) \operatorname{deg}(X)}{\left(4^{\ell}+1\right) \hat{h}(X)}},
$$

et le choix de $\ell$ que nous venons d'effectuer assure pour sa part,

$$
\left(4^{\ell}+1\right) \cdot \frac{\operatorname{deg}\left(\Phi_{\ell}(X)\right)}{h\left(\Phi_{\ell}(X)\right)} \leq 2 \cdot \frac{\operatorname{deg}(X)}{\hat{h}(X)} .
$$

Par ailleurs, toujours à l'aide de la proposition 3.14, on a :

$$
\frac{h\left(\Phi_{\ell}(X)\right)}{\operatorname{deg}\left(\Phi_{\ell}(X)\right)}>\frac{4^{\ell}+1}{2} \frac{\hat{h}(X)}{\operatorname{deg}(X)} \geq 3(4 \mathrm{e})^{d+2}(d+1) \frac{c_{0}(\Theta)}{2} ;
$$

en remplaçant finalement $c_{0}(\Theta)$ par sa valeur numérique (donnée à la proposition 3.9 ), on en déduit que l'hypothèse du lemme 5.1 est satisfaite. 
Comme par ailleurs une hypersurface de $\mathbb{P}_{N^{\prime}}$ de degré $L$ découpe sur $A$ le même diviseur qu'une hypersurface de degré $L^{\prime}=L .\left(4^{\ell}+1\right)$ de $\mathbb{P}_{N}$, le diviseur $Z$ de $\Phi_{\ell}(X)$ dont l'existence est assurée par le lemme 5.1, nous fournit, une fois tiré en arrière sur $\mathbb{P}_{N}$ un diviseur de $X$ (que nous noterons encore $Z$ ) dont le degré $\operatorname{deg}(Z)$ est bien majoré par la quantité :

$$
\begin{aligned}
\operatorname{deg}(Z) & \leq L\left(4^{\ell}+1\right) \operatorname{deg}(X) \\
& \leq 3(N+1)^{2} \cdot \max \left\{4^{\ell}+1 ; \operatorname{deg}(X)\right\} \cdot\left(4^{\ell}+1\right) \operatorname{deg}(X) \\
& \leqslant 3(N+1)^{2} \cdot \max \left\{1 ; \frac{12(4 \mathrm{e})^{d+2} \cdot(d+1) c_{0}(\Theta)}{\hat{h}(X)}\right\}^{2} \cdot \operatorname{deg}(X)^{3} \\
& \leqslant \frac{\delta^{2} \cdot \operatorname{deg}(X)}{3(N+1)^{2}} .
\end{aligned}
$$

Ainsi, toujours par le lemme 5.1, en tirant la situation en arrière sur $X$ et en tenant compte de la proposition 3.14 pour se ramener à des hauteurs normalisées, on voit que si $x$ est un point de $X$ de hauteur normalisée $<(4 \mathrm{e})^{-d-2} \cdot \frac{\hat{h}(X)}{\operatorname{deg}(X)}$, alors (rappelons que $\left.\left(4^{\ell}+1\right) \hat{h}(x)=\hat{h}\left(\Phi_{\ell}(x)\right)\right)$ :

$$
\begin{aligned}
h\left(\Phi_{\ell}(x)\right) & <(4 \mathrm{e})^{-d-2} \cdot \frac{\left(4^{\ell}+1\right) \hat{h}(X)}{\operatorname{deg}(X)}+2 c_{0}(\Theta) \\
& \leq(4 \mathrm{e})^{-d-2} \cdot \frac{h\left(\Phi_{\ell}(X)\right)}{\operatorname{deg}\left(\Phi_{\ell}(X)\right)}+2 c_{0}(\Theta)\left(1+\frac{d+1}{(4 \mathrm{e})^{d+2}}\right) .
\end{aligned}
$$

En tenant compte de la minoration obtenue en (22) pour $\frac{h\left(\Phi_{\ell}(X)\right)}{\operatorname{deg}\left(\Phi_{\ell}(X)\right)}$ et de la valeur numérique fixée pour $\ell$, on vérifie bien que

$$
h\left(\Phi_{\ell}(x)\right)<\frac{1}{(4 \mathrm{e})^{d+1}} \times \frac{h\left(\Phi_{\ell}(X)\right)}{\operatorname{deg}\left(\Phi_{\ell}(X)\right)}
$$

et le lemme 5.1 nous montre bien qu'en particulier $x$ est un élément du diviseur de $Z$ de $X$ introduit ci-dessus.

On en déduit les premières majorations de la proposition. Les majorations supplémentaires s'obtiennent tout simplement en tenant compte de la minoration obtenue au théorème 1.1 pour $\hat{h}(X)$. Ce qui démontre donc la proposition 5.2.

En ce qui concerne les minimums introduits au paragraphe 1, pour avoir un contrôle des ensembles exceptionnels, il est utile d'introduire des minimums « quantitatifs » et donc de restreindre les variations à des variétés de degré contrôlé. Plus précisément, on pose pour tout triplet d'entiers $\left(d, \Delta, \Delta^{\prime}\right)$ avec $d \geq 1, \Delta \geq 1$ et $\Delta^{\prime} \geq 1:$

$$
\hat{\mu}^{\text {ess }}\left(d, \Delta, \Delta^{\prime}\right):=\inf _{V \subset A} \sup _{Z \subset V} \inf \{\hat{h}(x) ; x \in(V \backslash Z)(\overline{\mathbb{Q}})\},
$$


l'infimum étant pris sur toutes les sous-variétés $V$ de $A$ définies sur $\overline{\mathbb{Q}}$, de dimensions $\leq d$, et de degrés majorés par $\Delta$, qui ne sont pas translatées de sous-variétés abeliennes, le supremum (qui est un maximum) est pour sa part pris sur les sousensembles algébriques propres de $V$ de degrés $\leq \Delta^{\prime}$ également définis sur $\overline{\mathbb{Q}}$.

De même, on introduit :

$$
\hat{\mu}^{\circ}\left(d, \Delta, \Delta^{\prime}\right):=\inf _{V \subset A} \sup _{Z \subset V^{\circ}} \inf \left\{\hat{h}(x) ; x \in\left(V^{\circ} \backslash Z\right)(\overline{\mathbb{Q}})\right\},
$$

le premier infimum étant pris sur toutes les sous-variétés $V$ de $A$ définies sur $\overline{\mathbb{Q}}$, de dimensions $\leq d$, de degrés majorés par $\Delta$, et le supremum $^{13}$ (qui est encore un maximum) est pour sa part pris sur les sous-ensembles finis de $V^{\circ}$ également définis sur $\overline{\mathbb{Q}}$ et de cardinaux au plus $\Delta^{\prime}$.

On déduit alors de la proposition 5.2 :

Théorème 5.3. Supposons donnés des entiers $d \geq 1$ et $\Delta \geq 1$, et posons :

$$
f(\Delta)=\left(2^{12 g+4} \cdot c_{0}(\Theta) C(A)\right)^{2} \cdot \Delta^{4 g^{2}} .
$$

On a alors les minorations suivantes pour les quantités $\mu^{\mathrm{ess}}(\cdot, \cdot, \cdot)$ et $\mu^{\circ}(\cdot, \cdot, \cdot)$ :

$$
\hat{\mu}^{\text {ess }}(d, \Delta, f(\Delta)) \geqslant \frac{3(N+1)^{2} \cdot c_{0}(\Theta)}{\sqrt{f(\Delta)}} \geqslant \frac{1}{f(\Delta)},
$$

ainsi que

$$
\hat{\mu}^{\circ}\left(d, \Delta, f^{\circ d}(\Delta)\right) \geqslant \frac{1}{f^{\circ d}(\Delta)} .
$$

Démonstration. Tout d'abord, on vérifie aisément, en tenant compte des valeurs numériques de $C(A)$ (donnée dans la proposition 5.2) et de $c_{0}(\Theta)$ (donnée à la proposition 3.9) :

$$
\frac{3(N+1)^{2} c_{0}(\Theta)}{\sqrt{f(\Delta)}} \geq \frac{1}{f(\Delta)} .
$$

Maintenant, si $V$ est une sous-variété de $A$, définie sur $\overline{\mathbb{Q}}$ de $\operatorname{degré} \operatorname{deg}(V) \leq \Delta$, et si $V$ n'est pas un translaté d'une sous-variété abelienne de $A$, la proposition 5.2 nous assure que les points de $V$ de hauteur au plus

$$
\mu:=\frac{1}{(4 \mathrm{e})^{d+2} C(A) \Delta^{2 k(b+1)}}
$$

appartiennent à un diviseur $Z$ de $V$, de degré au plus

$$
\operatorname{deg}(Z) \leq \tau:=3(N+1)^{2}\left[12(d+1)(4 \mathrm{e})^{d+2} c_{0}(\Theta) C(A)\right]^{2} \operatorname{deg}(V)^{4 k(b+1)+1},
$$

où, pour alléger, nous avons posé $k:=k_{V}$ et $b:=b_{V}$.

\footnotetext{
$\overline{13}$ Par convention l'infimum (respectivement le supremum) de l'ensemble vide est $+\infty$ (respectivement 0).
} 
Par définition de $\hat{\mu}^{\text {ess }}(d, \Delta, \cdot)$, on en déduit :

$$
\hat{\mu}^{\mathrm{ess}}(d, \Delta, \tau) \geq \mu
$$

comme il découle immédiatement de la définition de $\hat{\mu}^{\text {ess }}(d, \Delta, \cdot)$ que $\tau^{\prime} \geq \tau$ entraîne que $\hat{\mu}^{\text {ess }}\left(d, \Delta, \tau^{\prime}\right) \geq \hat{\mu}^{\text {ess }}(d, \Delta, \tau)$, il suffit de montrer d'une part que :

$$
\mu \geq \frac{3(N+1)^{2} c_{0}(\Theta)}{\sqrt{f(\Delta)}},
$$

et d'autre part que $f(\Delta) \geq \tau$ pour obtenir la première partie du théorème.

Commençons par la première inégalité ; on remarque aisément que :

$$
2 k(b+1) \leqslant 2(g-1)(g+1)<2 g^{2},
$$

il suffit maintenant de vérifier que :

$$
\frac{1}{(4 \mathrm{e})^{d+2} C(A)} \geq \frac{3(N+1)^{2}}{2^{12 g+4} C(A)},
$$

qui est triviale en tenant compte de $N+1=16^{g}, d \leq g-1$ et $g \geq 2$ (le théorème est vide si $g=1$ ).

Pour vérifier que $\tau \leq f(\Delta)$, il suffit, en tenant compte de nouveau de l'inégalité $2 k(b+1)<2 g^{2}$ et en remplaçant $f(\Delta)$ par sa valeur, de vérifier que

$$
432(N+1)^{2}(d+1)^{2}(4 \mathrm{e})^{2 d+4} \leq 2^{24 g+8},
$$

qui est triviale.

Vérifions maintenant que

$$
\hat{\mu}^{\circ}\left(d, \Delta, f^{\circ d}(\Delta)\right) \geq \frac{1}{f^{\circ d}(\Delta)} .
$$

Pour ceci, nous allons commencer par remarquer que, si $d \geqslant 2$ :

$$
\hat{\mu}^{\circ}\left(d, \Delta, f^{\circ d}(\Delta)\right) \geq \min \left\{\hat{\mu}^{\text {ess }}(d, \Delta, f(\Delta)) ; \hat{\mu}^{\circ}\left(d-1, f(\Delta), f^{\circ d}(\Delta)\right)\right\} .
$$

Par définition des $\hat{\mu}^{\circ}(\cdot, \cdot, \cdot)$, pour tout $\varepsilon>0$, il existe une sous-variété $V$ de $A$ de dimension $d$ et de degré au plus $\Delta$, qui est définie sur $\overline{\mathbb{Q}}$ et qui n'est pas translatée d'une sous-variété abelienne de $A$, telle que :

$$
\hat{\mu}^{\circ}\left(d, \Delta, f^{\circ d}(\Delta)\right) \geqslant \inf \left\{\hat{h}(x), x \in\left(V^{\circ} \backslash\left(V^{\circ} \cap Z\right)\right)(\overline{\mathbb{Q}})\right\}-\varepsilon
$$

pour tout ensemble fini $Z$ d'au plus $f^{\circ d}(\Delta)$ points de $A$.

Il existe un diviseur $W$ de degré $\leq f(\Delta)$ tel que

$$
\inf \{\hat{h}(x) ; x \in(V \backslash W)(\overline{\mathbb{Q}})\} \geq \hat{\mu}^{\mathrm{ess}}(d, \Delta, f(\Delta))-\varepsilon,
$$

et il existe un ensemble fini $Z$ d'au plus $f^{\circ d}(\Delta)$ points de $W^{\circ}$ tel que

$$
\inf \left\{\hat{h}(x) ; x \in\left(W^{\circ} \backslash Z\right)(\overline{\mathbb{Q}})\right\} \geq \hat{\mu}^{\circ}\left(d-1, f(\Delta), f^{\circ d}(\Delta)\right)-\varepsilon .
$$


On a alors

$$
\begin{aligned}
\hat{\mu}^{\circ}\left(d, \Delta, f^{\circ d}(\Delta)\right) & \geq \min \left\{\inf \left\{\hat{h}(x) ; x \in\left(W^{\circ} \backslash Z\right)(\overline{\mathbb{Q}})\right\}\right. \\
& \inf \{\hat{h}(x) ; x \in(V \backslash W)(\overline{\mathbb{Q}})\}\}-\varepsilon \\
& \geq \min \left\{\hat{\mu}^{\circ}\left(d-1, f(\Delta), f^{\circ d}(\Delta)\right) ; \hat{\mu}^{\text {ess }}(d, \Delta, f(\Delta))\right\}-2 \varepsilon
\end{aligned}
$$

car $W^{\circ} \supset V^{\circ} \cap W$. L'inégalité (23) s'en déduit en faisant tendre $\varepsilon$ vers 0 .

On déduit par récurrence de la relation (23), lorsque $j=1, \ldots, d-1$,

$$
\hat{\mu}^{\circ}\left(d, \Delta, f^{\circ d}(\Delta)\right) \geq \min _{1 \leq j \leq d}\left\{\hat{\mu}^{\text {ess }}\left(d-j+1, f^{\circ(j-1)}(\Delta), f^{\circ j}(\Delta)\right)\right\},
$$

$\operatorname{car} \hat{\mu}^{\circ}(1, \cdot, \cdot)=\hat{\mu}^{\text {ess }}(1, \cdot, \cdot)$.

La première partie de la preuve nous donne donc :

$$
\hat{\mu}^{\circ}\left(d, \Delta, f^{\circ d}(\Delta)\right) \geq \min \left\{\frac{1}{f(\Delta)}, \frac{1}{f^{\circ 2}(\Delta)}, \ldots, \frac{1}{f \circ d(\Delta)}\right\}=\frac{1}{f^{\circ d}(\Delta)} .
$$

Le théorème 5.3 est donc entièrement établi.

Démonstration des théorèmes 1.2, 1.3 et 1.4. Pour établir le théorème 1.2, il reste à évaluer la constante.

Posons $C^{\prime}(A):=\left(2^{12 g+4} \cdot c_{0}(\Theta) C(A)\right)^{1 / 2 g^{2}} ;$ par définition de $f$, on a $f(\Delta)=$ $\left(C^{\prime}(A) \Delta\right)^{4 g^{2}}$ et l'on trouve

$f^{\circ d}(\Delta)=\left(C^{\prime}(A) f^{\circ(d-1)}(\Delta)\right)^{4 g^{2}}=C^{\prime}(A)^{4 g^{2}+\cdots+\left(4 g^{2}\right)^{d}} \cdot \Delta^{\left(4 g^{2}\right)^{d}} \leq\left(C^{\prime}(A)^{2} \cdot \Delta\right)^{\left(4 g^{2}\right)^{d}}$.

Comme $C^{\prime}(A)^{2 g^{2}} \leq 2^{14 g^{3}} \max \{1, h(A)\} \cdot \min \left\{1 ; \mathcal{R}_{\text {inj }}\right\}^{-2(g+1)}$, cette dernière quantité est bien majorée par $q(X)$ lorsque $d=\operatorname{dim}(X)$ et $\Delta=\operatorname{deg}(X)$, ce qui achève de montrer le théorème 1.2 .

Pour le théorème 1.3 on raisonne comme suit (voir aussi [Ré], preuve du théorème 2.1 et lemme 6.1 ).

Soient $x_{1}, \ldots, x_{r}$ des éléments de $\Gamma$, dont les projections sur $\Gamma / \Gamma_{\text {tors }}$ engendrent $\Gamma / \Gamma_{\text {tors }}$; tout élément $x \in \Gamma$ s'écrit donc de façon unique ${ }^{14} x=\alpha_{1} x_{1}+\cdots+\alpha_{r} x_{r}+t$, où $t \in \Gamma_{\text {tors }}$, et la hauteur $\hat{h}(x)$ est la valeur en $\boldsymbol{\alpha}=\left(\alpha_{1}, \ldots, \alpha_{r}\right) \in \mathbb{Q}^{r}$ d'une forme quadratique définie positive $q$. Soit $\varepsilon: \Gamma \rightarrow \mathbb{R}^{r}$ définie par $\varepsilon(x)=\boldsymbol{\alpha}$. Ainsi, pour tout nombre réel $a \geq 0$, les images par $\varepsilon$ de l'ensemble des points $x \in \Gamma$ satisfaisant $\hat{h}(x) \leqslant a$ sont contenues dans un ellipsoïde $\mathcal{E}(a)$ de $\mathbb{R}^{r}$. Posons $a_{0}:=f^{\circ d}(\Delta)^{-1}$ et recouvrons $\mathcal{E}(a) \cap \Gamma \cap X^{\circ}(\overline{\mathbb{Q}})$ par des l'ellipsoïdes translatés de

\footnotetext{
$\overline{14}$ Il est sous-entendu que pour chaque entier $n \geq 1$ et chaque indice $i, 1 \leq i \leq r$, un point de $n$-division de $x_{i}$ est fixé une fois pour toutes; ce dernier est noté $x_{i} / n$.
} 
la forme $\left\{x_{i}^{\prime}+\mathcal{E}\left(a_{0}\right)\right\}_{i \in I}$ de telle sorte que les ellipsoïdes $x_{i}^{\prime}+\mathcal{E}\left(\frac{a_{0}}{4}\right)$ soient deux à deux disjoints. On peut choisir $x_{i}^{\prime} \in \mathcal{E}(a) \cap \Gamma \cap X^{\circ}(\overline{\mathbb{Q}})$ pour $i \in I$; le nombre d'ellipsoïdes du recouvrement (i. e. le cardinal de l'ensemble d'indices $I$ ) nécessaires pour cette opération est majoré par le rapport des volumes des ellipsoïdes $\mathcal{E}\left(\frac{a_{0}}{4}\right)$ et $\mathcal{E}\left(\left(\sqrt{a}+\frac{1}{2} \sqrt{a_{0}}\right)^{2}\right)$ qui contient l'union des $x_{i}^{\prime}+\mathcal{E}\left(\frac{a_{0}}{4}\right)$

$$
\operatorname{Vol}\left(\mathcal{E}\left(\left(\sqrt{a}+\frac{1}{2} \sqrt{a_{0}}\right)^{2}\right)\right) / \operatorname{Vol}\left(\mathcal{E}\left(\frac{a_{0}}{4}\right)\right)=\left(2 \sqrt{\frac{a}{a_{0}}}+1\right)^{r} .
$$

Maintenant, dans chacun des ensembles $\varepsilon^{-1}\left(x_{i}^{\prime}+\mathcal{E}\left(a_{0}\right)\right)$, il y a au plus $f^{\circ d}(\Delta)$ points de $\Gamma \cap X^{\circ}(\overline{\mathbb{Q}})$, en vertu du théorème 5.3. En effet, pour tout élément $v$ de $\varepsilon^{-1}\left(x_{i}^{\prime}+\mathcal{E}\left(a_{0}\right)\right) \cap \Gamma \cap X^{\circ}(\overline{\mathbb{Q}})$ on a $v-x_{i}^{\prime} \in \Gamma \cap\left(\tau_{-x_{i}^{\prime}}(X)\right)^{\circ} \cap \varepsilon^{-1}\left(\mathcal{E}\left(a_{0}\right)\right)$. En particulier, $v-x_{i}^{\prime}$ est un élément de $\left(\tau_{-x_{i}^{\prime}}(X)\right)^{\circ}$ de hauteur au plus $1 / f^{\circ d}(\Delta)$ et le théorème 5.3 nous assure qu'il y a au plus $f^{\circ d}(\Delta)$ tels points puisque

$$
\operatorname{deg}\left(\tau_{-x_{i}^{\prime}}(X)\right)=\operatorname{deg}(X) .
$$

Au total, il y a donc au plus

$$
\operatorname{card}(I) f^{\circ d}(\Delta) \leq\left(2 \sqrt{\frac{a}{a_{0}}}+1\right)^{r} f^{\circ d}(\Delta) \leq\left(5 a f^{\circ d}(\Delta)\right)^{r / 2} f^{\circ d}(\Delta)
$$

points dans l'ensemble $\left\{x \in \Gamma \cap X^{\circ}(\overline{\mathbb{Q}}) ; \hat{h}(x) \leq a\right\}$; pour justifier l'inégalité cidessus, il suffit de vérifier que $\left(2 \sqrt{a / a_{0}}+1\right)^{2} \leq 5 a f^{\circ d}(\Delta)$, c'est-à-dire

$$
4 \sqrt{a / a_{0}}+1 \leq a f^{\circ d}(\Delta),
$$

et cette inégalité découle trivialement du fait que $a \geq 1$ et de la relation $f^{\circ d}(\Delta) \geq$ $2^{28}$ car, par définition, $c_{0}(\Theta) \geq 1$ ainsi que $C(A) \geq 1$.

Le théorème 1.3 est donc entièrement établi.

Pour le théorème 1.4 on reporte la minoration de $\mathcal{R}_{\text {inj }}$, obtenue au lemme 6.8 de l'appendice, dans l'expression de $q(X)$ et le théorème 1.1. On a, dans les notations du théorème $1.4, \mathcal{R}_{\text {inj }} \geq g^{-2} . h_{0}(A)^{-1 / 2}$ et donc

$$
\begin{aligned}
q(X) & \leq\left(g^{4(g+1)} \cdot h_{0}(A)^{g+2}\right)^{4\left(4 g^{2}\right)^{\operatorname{dim}(X)-1}} \cdot\left(2^{14 g} \operatorname{deg}(X)\right)^{\left(4 g^{2}\right)^{\operatorname{dim}(X)}} \\
& \leq\left(g^{4(g+1) / g^{2}} \cdot 2^{14 g} \cdot h_{0}(A)^{(g+2) / g^{2}} \cdot \operatorname{deg}(X)\right)^{\left(4 g^{2}\right)^{\operatorname{dim}(X)}} \\
& \leq\left(2^{16 g} \cdot h_{0}(A)^{2 / g} \cdot \operatorname{deg}(X)\right)^{\left(4 g^{2}\right)^{\operatorname{dim}(X)}}
\end{aligned}
$$

puis, lorsque $X$ n'est pas translatée d'une sous-variété abelienne, pour le théorème 1.1

$$
\begin{aligned}
\frac{\hat{h}(X)}{\operatorname{deg}(X)} & \geq g^{-4(g+1)} 2^{-9 g^{3}} \cdot h_{0}(A)^{-b-1} \operatorname{deg}(X)^{-2 k(b+1)} \\
& \geq 2^{-11 g^{3}} \cdot \operatorname{deg}(X)^{-2 k(b+1)} \cdot h_{0}(A)^{-b-1},
\end{aligned}
$$

ce qui conduit bien au théorème 1.4. 


\section{Appendice}

L'objet de cet appendice est de donner une version explicite d'un énoncé maintenant classique : le « lemme matriciel » de D. MASSER (voir [Ma], page 115); on en déduit facilement une estimation du rayon d'injectivité $\mathcal{R}_{\text {inj. }}$. Pour ceci, nous commençons par donner une minoration de la norme archimédienne de l'origine d'une variété abelienne dans le plongement thêta; nous passerons ensuite à une preuve de ce lemme.

Nous rappelons tout d'abord quelques notations, définitions et lemmes classiques de la théorie de la réduction des matrices symétriques, dont nous donnons au passage des versions quantitatives.

Soit $g$ un entier positif, on notera $\mathfrak{S}_{g}$ l'espace de SIEGEL en dimension $g$ i. $e$. l'espace des matrices $g \times g$, symétriques, de partie imaginaire définie positive; on notera $\mathcal{S}_{g}$ l'espace des matrices $g \times g$ symétriques, réelles et enfin $\mathfrak{P}_{g}$ le sousensemble de $\mathcal{S}_{g}$ formé des matrices définies positives. Tous les vecteurs considérés seront notés en colonnes et la transposée sera notée $~{ }^{t} \gg ;$ si $n$ est un entier $\geq 1$, on notera $\mathcal{Z}_{n}$ l'ensemble $\frac{1}{n} \mathbb{Z}^{g} / \mathbb{Z}^{g}$. Sauf précision du contraire, la norme d'une matrice sera la norme du sup.

Rappelons que le groupe symplectique $\operatorname{Sp}_{2 g}(\mathbb{Z})$ de dimension $2 g$ agit sur $\mathfrak{S}_{g}$ de la façon suivante : soit $\sigma$ un élément de $\operatorname{Sp}_{2 g}(\mathbb{Z})$ et $\tau$ un élément de $\mathfrak{S}_{g}$; alors $\sigma \cdot \tau$ est défini par :

$$
\sigma \cdot \tau=(\alpha \tau+\beta)(\gamma \tau+\delta)^{-1}, \quad \text { où } \quad \sigma=\left(\begin{array}{cc}
\alpha & \beta \\
\gamma & \delta
\end{array}\right) .
$$

Définition 6.1. Soit $\tau=x+\mathrm{i} y$ un élément de l'espace de Siegel $\mathfrak{S}_{g}=\mathcal{S}_{g}+\mathrm{i} \mathfrak{P}_{g}$, on dit que $\tau$ est Siegel réduite si :

(i) $\forall i, j, 1 \leq i, j \leq g,\left|x_{i, j}\right| \leq \frac{1}{2}$;

(ii) $\forall \sigma \in \operatorname{Sp}_{2 g}(\mathbb{Z}), \operatorname{det}(\Im m(\sigma \cdot \tau)) \leq \operatorname{det}(\Im m(\tau))$;

(iii) la partie imaginaire $y$ de $\tau$ est Minkowski réduite, $i$ e. :

(1) pour tout élément $\xi=\left(\xi_{1}, \ldots, \xi_{g}\right) \in \mathbb{Z}^{g}$ et pour tout indice $k, 1 \leq k \leq$ $g$, tel que les nombres $\xi_{k}, \ldots, \xi_{g}$ sont non tous nuls, on a ${ }^{t} \xi y \xi \geq y_{k}:=$ $y_{k, k}$

(2) pour tout $k, 1 \leq k \leq g-1$, on a $y_{k, k+1} \geq 0$.

On notera $\mathfrak{F}_{g}$ l'ensemble des matrices Siegel réduites. On rappelle que $\mathfrak{F}_{g}$ est « un domaine fondamental » de $\mathfrak{S}_{g}$ pour l'action de $\mathrm{Sp}_{2 g}(\mathbb{Z})$.

Lemme 6.2. Soit $K$ un corps, $g$ un entier $\geq 1$ et $y$ une matrice $g \times g$, symétrique. Soit enfin $1 \leq g^{\prime} \leq g$ un entier tel que le mineur principal $y^{\prime}$ d'ordre $g^{\prime} \times g^{\prime}$ de $y$ soit inversible. Dans ces conditions, l'équation:

$$
y=\left(\begin{array}{ll}
\operatorname{Id}_{g^{\prime}} & w \\
0 & \operatorname{Id}_{g^{\prime \prime}}
\end{array}\right)\left(\begin{array}{ll}
y^{\prime} & 0 \\
0 & y^{\star}
\end{array}\right)\left(\begin{array}{ll}
\operatorname{Id}_{g^{\prime}} & w \\
0 & \operatorname{Id}_{g^{\prime \prime}}
\end{array}\right),
$$


d'inconnues $y^{\star} \in M_{g^{\prime \prime}}(K), w \in M_{g^{\prime} \times g^{\prime \prime}}(K)$ admet une solution unique (bien entendu, ici, $\left.g^{\prime \prime}=g-g^{\prime}\right)$; de plus, $y^{\star}$ est symétrique.

Démonstration. Voir par exemple [Ig], lemme 12, page 190.

Lemme 6.3. (《théorème d'Hermite ») Soient g un entier $\geq 1$, y un élément de $\mathfrak{P}_{g}$ et $m(y)$ le minimum de la forme quadratique associée sur le réseau $\mathbb{Z}^{g}$ privé de l'origine. On a:

$$
m(y)^{g} \leq\left(\frac{4}{3}\right)^{\frac{1}{2} g(g-1)} \operatorname{det}(y) .
$$

Démonstration. Le lemme est clairement vrai pour $g=1$, supposons-le donc vrai pour $g-1 \geq 1$, et vérifions-le pour $g$. Soit $u_{1} \in \mathbb{Z}^{g}$ tel que $m(y)={ }^{t} u_{1} y u_{1}$. Les coefficients de $u_{1}$ étant premiers entre eux, on peut compléter $u_{1}$ en un élément $u$ de $\mathrm{Gl}_{g}(\mathbb{Z})$ ayant $u_{1}$ comme première colonne. En remplaçant $y$ par ${ }^{t} u y u$, on peut supposer que $y_{1}=y_{1,1}=m(y)$. Appliquons maintenant le lemme 6.2 avec $g^{\prime}=1$. On en déduit que pour tout $\xi=\left(\xi^{\prime}, \xi^{\prime \prime}\right) \in \mathbb{R}^{g}$ (avec $\left.\xi^{\prime} \in \mathbb{R}\right)$, on a :

$$
{ }^{t} \xi y \xi=y_{1}\left(\xi^{\prime}+w \xi^{\prime \prime}\right)^{2}+{ }^{t} \xi^{\prime \prime} y^{\star} \xi^{\prime \prime} .
$$

Choisissons maintenant $\xi^{\prime \prime} \in \mathbb{Z}^{g-1}$ tel que ${ }^{t} \xi^{\prime \prime} y^{\star} \xi^{\prime \prime}=m\left(y^{\star}\right)$, et $\xi^{\prime} \in \mathbb{Z}$ tel que $\xi^{\prime}+w \xi^{\prime \prime}$ soit minimal en valeur absolue, on en tire donc :

$$
y_{1}=m(y) \leq{ }^{t} \xi y \xi \leq \frac{1}{4} y_{1}+m\left(y^{\star}\right),
$$

i. e. $y_{1} \leq \frac{4}{3} m\left(y^{\star}\right)$. Cette inégalité, jointe à l'hypothèse de récurrence donne donc :

$$
\begin{aligned}
m(y)^{g} & \leq\left(\frac{4}{3}\right)^{g-1} y_{1} m\left(y^{\star}\right)^{g-1} \\
& \leq\left(\frac{4}{3}\right)^{(g-1)+\frac{(g-1)(g-2)}{2}} y_{1} \operatorname{det}\left(y^{\star}\right)=\left(\frac{4}{3}\right)^{\frac{g(g-1)}{2}} \operatorname{det}(y) .
\end{aligned}
$$

Le lemme 6.3 est établi.

Lemme 6.4. Soit y une matrice $g \times g$ Minkowski réduite. On a :

(i)

$$
\operatorname{det}(y) \leq y_{1} \ldots y_{g} \quad \text { et } \quad y_{1} \ldots y_{g} \leq c_{5} \operatorname{det}(y)
$$

où l'on peut prendre : $c_{5}:=\left(\frac{2 g(g-1)}{3}\right)^{\frac{1}{2} g(g-1)}$;

(ii) pour tout $\xi=\left(\xi_{1}, \ldots, \xi_{g}\right) \in \mathbb{R}^{g}$, on $a$ :

$$
{ }^{t} \xi y \xi \leq \frac{g+1}{2} \sum_{i=1}^{g} y_{i} \xi_{i}^{2} \quad \text { et } \quad \sum_{i=1}^{g} y_{i} \xi_{i}^{2} \leq c_{6}{ }^{t} \xi y \xi,
$$

où l'on peut prendre $: c_{6}:=\left(\frac{2 g(g-1)}{3}\right)^{\frac{1}{2} g(g-1)} \cdot\left(\frac{g+1}{2}\right)^{g-1}$. 
Démonstration. La première inégalité du point (i) est le « théorème de HADAMARD » (voir par exemple [Ig], page 190). Il suffit donc d'établir la deuxième. Clairement, l'énoncé est vrai pour $g=1$ (avec $c_{5}=1$ ) et pour $g=2$ (avec $c_{5}=\frac{4}{3}$ : voir $[\mathrm{Ig}]$, page 192). Il suffit donc de faire une récurrence. Soit $g \geq 3$ et $y$ une matrice Minkowski réduite de dimension $g$. Supposons que pour tout $k$, $1 \leq k \leq g-1$, on ait : $y_{k+1} \leq \alpha y_{k}$, avec $\alpha=\frac{g(g-1)}{4}$. On a alors par le lemme 6.3 :

$$
y_{1} \ldots y_{g} \leq \alpha^{\frac{g(g-1)}{2}} y_{1}^{g} \leq \alpha^{\frac{g(g-1)}{2}}\left(\frac{4}{3}\right)^{\frac{1}{2} g(g-1)} \operatorname{det}(y)
$$

et le lemme est vrai. Nous pouvons donc supposer qu'il existe un entier $k, 1 \leq k \leq$ $g-1$, tel que $y_{i+1} \leq \alpha y_{i}$ pour tout $i, k+1 \leq i \leq g-1$, et $y_{k+1}>\alpha y_{k}$. Appliquons le lemme 6.2 avec $g^{\prime}=k$. Choisissons $\xi^{\prime \prime} \in \mathbb{Z}^{g-k}$ tel que ${ }^{t} \xi^{\prime \prime} y^{\star} \xi^{\prime \prime}=m\left(y^{\star}\right)$ et $\xi^{\prime} \in \mathbb{Z}^{k}$ tel que toutes les coordonnées de $\xi^{\star}=\xi^{\prime}+w \xi^{\prime \prime}$ soient $\leq \frac{1}{2}$, et posons $\xi=\left(\xi^{\prime}, \xi^{\prime \prime}\right)$. Puisque $y$ est Minkowski réduite, (définition 6.1, propriété (iii), point 1), on a $y_{k+1} \leq{ }^{t} \xi y \xi$ et l'on en tire :

$$
y_{k+1} \leq{ }^{t} \xi y \xi={ }^{t} \xi^{\star} y^{\prime} \xi^{\star}+m\left(y^{\star}\right) \leq \frac{k(k+1)}{8} y_{k}+\left(\frac{4}{3}\right)^{\frac{g-k-1}{2}}\left(\operatorname{det}\left(y^{\star}\right)\right)^{\frac{1}{g-k}} .
$$

On tire de cette inégalité et de l'inégalité $y_{k+1}>\alpha y_{k}$,

$$
y_{k+1} \leq \frac{1}{1-\frac{k(k+1)}{2 g(g-1)}}\left(\frac{4}{3}\right)^{\frac{1}{2}(g-k-1)}\left(\operatorname{det}\left(y^{\star}\right)\right)^{\frac{1}{g-k}}
$$

Comme $\frac{k(k+1)}{2 g(g-1)} \leq \frac{1}{2}$, l'hypothèse de récurrence donne alors :

$$
\begin{aligned}
y_{1} \ldots y_{g} & =\left(y_{1} \ldots y_{k}\right)\left(y_{k+1} \ldots y_{g}\right) \\
& \leq\left(\frac{2 k(k-1)}{3}\right)^{\frac{k(k-1)}{2}} \cdot \operatorname{det}\left(y^{\prime}\right) \alpha^{\frac{(g-k)(g-k-1)}{2}} y_{k+1}^{g-k} \\
& \leq 2^{g-k}\left(\frac{g(g-1)}{4}\right)^{\frac{(g-k)(g-k-1)}{2}}\left(\frac{2 k(k-1)}{3}\right)^{\frac{k(k-1)}{2}} \operatorname{det}(y) \\
& \leq\left(\frac{g(g-1)}{3}\right)^{\frac{(g-k)(g-k-1)+k(k-1)}{2}} 2^{g-k+\frac{k(k-1)}{2}} \operatorname{det}(y) \\
& \leq\left(\frac{2 g(g-1)}{3}\right)^{\frac{1}{2} g(g-1)} \operatorname{det}(y)
\end{aligned}
$$

Le point (i) du lemme 6.4 est donc établi. Passons à (ii).

Soit $d$ la matrice diagonale dont le $i^{\text {ème }}$ coefficient est $d_{i}=\sqrt{y_{i}}$, et $y^{\star}=$ $d^{-1} y d^{-1}$. Comme $\left|y_{i, j}^{\star}\right|=\frac{\left|y_{i, j}\right|}{d_{i} d_{j}} \leq \frac{1}{2}$, pour $i \neq j$ (et $y_{i, i}^{\star}=1$ ), la plus grande valeur propre de $y^{\star}$ est $\leq \frac{g+1}{2}$; par ailleurs, $\operatorname{det}\left(y^{\star}\right)=\frac{\operatorname{det}(y)}{y_{1} \ldots y_{g}}$ et le point (i) nous assure 
que $c_{5}^{-1} \leq \operatorname{det}\left(y^{\star}\right) \leq 1$. On en tire que la plus petite valeur propre de $y^{\star}$ est $\geq\left(\frac{2}{g+1}\right)^{g-1} c_{5}^{-1} \geq c_{6}^{-1}$, ce qui montre bien le point (ii) et donc le lemme 6.4.

Rappelons que pour $p=(a, b) \in \mathbb{R}^{2 g}$ on a noté $\theta_{p}(\tau, z)$ la fonction thêta avec caractéristique classique (confer formule (10)).

Lemme 6.5. Pour tout $\tau \in \mathfrak{S}_{g}$, on a :

$$
\max \left\{\left|\theta_{p}(\tau, 0)\right|, p \in \mathcal{Z}_{2}^{2}\right\} \geq 1 .
$$

Démonstration. (Voir aussi [Ma-Wu2], lemme 6.3.) Soient $\tau \in \mathfrak{S}_{g}$ et $\tau^{\star} \in \mathfrak{F}_{g}$ tels que $\tau$ soit l'image de $\tau^{\star}$ via un élément $\sigma=\left(\begin{array}{ll}\alpha & \beta \\ \gamma & \delta\end{array}\right)$ de $\operatorname{Sp}_{2 g}(\mathbb{Z})$. Les formules de transformations modulaires (voir par exemple [Ig], page 85), nous donnent alors :

$$
\max \left\{\left|\theta_{p}(\tau, 0)\right|, p \in \mathcal{Z}_{2}^{2}\right\}=\left|\operatorname{det}\left(\gamma \tau^{\star}+\delta\right)\right|^{\frac{1}{2}} \max \left\{\left|\theta_{p}\left(\tau^{\star}, 0\right)\right|, p \in \mathcal{Z}_{2}^{2}\right\} .
$$

Or, comme $\tau^{\star} \in \mathfrak{F}_{g}$, on a $\left|\operatorname{det}\left(\gamma \tau^{\star}+\delta\right)\right| \geq 1$ (voir définition 6.1, point (ii)). Par ailleurs, en raisonnant comme dans [Da], $\S$. 3, on voit que :

$$
\lim _{n \longrightarrow \infty} \theta_{(0,0)}\left(2^{n} \tau^{\star}, 0\right)=1 \text {. }
$$

En effet,

$$
\theta_{(0,0)}\left(2^{n} \tau^{\star}, 0\right)=1+\sum_{m \in \mathbb{Z}^{g}, m \neq 0} \exp \left(\mathrm{i} \pi^{t} m 2^{n} \tau^{\star} m\right),
$$

Or, en vertu du lemme 6.4, point (ii), et comme $\tau^{\star}$ est supposée SiEgEL réduite,

$$
\lim _{n \longrightarrow \infty}\left|\sum_{m \in \mathbb{Z}^{g}, m \neq 0} \exp \left(\mathrm{i} \pi^{t} m 2^{n} \tau^{\star} m\right)\right| \leq \lim _{n \longrightarrow \infty} \sum_{m \in \mathbb{Z}^{g}, m \neq 0} \exp \left(-\frac{\pi 2^{n} \sqrt{3}}{2 c_{6}} \sum_{i=1}^{g} m_{i}^{2}\right)=0 .
$$

Les formules de «duplication » ([Ig], théorème 2, page 139) assurent que :

$$
\max \left\{\left|\theta_{p}\left(2^{n} \tau^{\star}, 0\right)\right|, p \in \mathcal{Z}_{2}^{2}\right\} \leq \max \left\{\left|\theta_{p}\left(2^{n-1} \tau^{\star}, 0\right)\right|, p \in \mathcal{Z}_{2}^{2}\right\},
$$

en effet, spécialisées au cas particulier qui nous intéresse, ces dernières s'écrivent :

$$
\theta_{(a, b)}\left(2^{n} \tau^{\star}, 0\right)^{2}=\frac{1}{2^{g}} \sum_{m \in \mathcal{Z}_{2}} \exp \left(-4 \mathrm{i} \pi^{t} a . m\right) \theta_{(2 a, b+m)}\left(2^{n-1} \tau^{\star}, 0\right) \theta_{(0, m)}\left(2^{n-1} \tau^{\star}, 0\right) .
$$

Ces inégalités mises ensemble donnent le lemme 6.5.

Lemme 6.6. Pour tout $\tau \in \mathfrak{F}_{g}$ et tout $b \in \mathcal{Z}_{2}$, on a, avec $e_{g}:={ }^{t}(0, \ldots, 0,1) \in$ $\mathbb{Z}^{g}:$

$$
\left|\theta_{\left(\frac{e g}{2}, b\right)}(\tau, 0)\right| \leq(3 g)^{\frac{g^{2}}{2}+g+\frac{3}{2}}\|\Im m \tau\|^{\frac{g-1}{2}} \mathrm{e}^{-\frac{\pi}{4}\|\Im m \tau\|} \leq(4 g)^{2 g^{2}} \mathrm{e}^{-\frac{\pi}{8}\|\Im m \tau\|} .
$$

De plus, il existe un élément $b \in \mathcal{Z}_{2}$ tel que l'on ait $0 \neq \theta_{\left(\frac{e_{g}}{2}, b\right)}(\tau, 0)$. 
Démonstration. L'existence d'un élément $b \in \mathcal{Z}_{2}$ tel que $0 \neq \theta_{\left(\frac{e_{g}}{2}, b\right)}(\tau, 0)$ découle des formules de duplication (voir [Ig], theorem 2, page 139); en effet, soit $z \in \mathbb{C}^{g}$, on a :

$\theta_{\left(\frac{e g}{2}, 0\right)}(2 \tau, z) \theta_{(0,0)}(2 \tau, z)=\frac{1}{2^{g}} \sum_{m \in \frac{1}{2} \mathbb{Z}^{g} / \mathbb{Z}^{g}} \exp \left(-\mathrm{i} \pi^{t} e_{g} \cdot m\right) \theta_{\left(\frac{e g}{2}, m\right)}(\tau, z) \theta_{\left(\frac{e g}{2}, m\right)}(\tau, 0) ;$

comme le membre de gauche n'est pas identiquement nul lorsque $z$ décrit $\mathbb{C}^{g}$, l'un $\operatorname{des} \theta_{\left(\frac{e g}{2}, m\right)}(\tau, 0)$ est non nul, d'où la deuxième partie du lemme 6.6 (voir aussi le lemme 11, page 168 de [Ig]).

Il suffit donc d'établir la majoration. Soit donc $\tau \in \mathfrak{F}_{g}$, et $b \in \mathcal{Z}_{2}$. On a :

$$
\left|\theta_{\left(\frac{e_{g}}{2}, b\right)}(\tau, 0)\right| \leq \sum_{m \in \mathbb{Z}^{g}} \exp \left(-\pi^{t}\left(m+\frac{e_{g}}{2}\right) \Im m \tau\left(m+\frac{e_{g}}{2}\right)\right)
$$

Pour évaluer la somme du membre de droite, notons $A_{k}$ le nombre :

$$
A_{k}:=\operatorname{card}\left\{m \in \mathbb{Z}^{g},{ }^{t}\left(2 m+e_{g}\right) y\left(2 m+e_{g}\right)<k^{2} y_{g}\right\}
$$

On a donc, avec la propriété (iii)-(1) des matrices SIEGEL-réduites, $A_{1}=0$ et

$$
\left|\theta_{\left(\frac{e g}{2}, b\right)}(\tau, 0)\right| \leq \sum_{k \geq 1} A_{k+1} \mathrm{e}^{-\frac{\pi k^{2} y_{g}}{4}}
$$

Remarquons que si $a$ est un nombre réel $\geq 1$,

$$
\begin{aligned}
\int_{x \geq a} x^{g} \mathrm{e}^{-x^{2}} d x & =\frac{a^{g-1} \mathrm{e}^{-a^{2}}}{2} \int_{0}^{\infty}\left(\frac{v}{a^{2}}+1\right)^{\frac{g-1}{2}} \mathrm{e}^{-v} d v \\
& \leq \frac{a^{g-1} \mathrm{e}^{-a^{2}}}{2} \int_{0}^{\infty}(v+1)^{\frac{g-1}{2}} \mathrm{e}^{-v} d v \\
& \leq \frac{1}{2} a^{g-1} \mathrm{e}^{-a^{2}+1} \Gamma\left(\frac{g+1}{2}\right) \\
& \leq 2\left(\frac{g+1}{2}\right)^{\frac{g}{2}} a^{g-1} \mathrm{e}^{-a^{2}} .
\end{aligned}
$$


Maintenant, en posant $a:=\max \left\{1 ; \sqrt{\frac{2 g}{\pi y_{g}}}\right\} \in[1, \sqrt{g}]$, on dispose de l'inégalité :

$$
\begin{aligned}
\sum_{k \geq 1} k^{g} \mathrm{e}^{-\frac{\pi k^{2} y_{g}}{4}} & \leq a^{g+1} \mathrm{e}^{-\frac{\pi a^{2} y_{g}}{4}}+\int_{x \geq a} x^{g} \mathrm{e}^{-\frac{\pi x^{2} y_{g}}{4}} d x \\
& \leq a^{g+1} \mathrm{e}^{-\frac{\pi a^{2} y_{g}}{4}}+\left(\frac{4}{\pi y_{g}}\right)^{\frac{g+1}{2}} \int_{v \geq \frac{a \sqrt{\pi y_{g}}}{2}} v^{g} \mathrm{e}^{-v^{2}} d v \\
& \leq a^{g+1} \mathrm{e}^{-\frac{\pi a^{2} y_{g}}{4}}\left(1+\left(\frac{g+1}{2}\right)^{\frac{g}{2}} \cdot \frac{4}{g}\right) \\
& \leq 4\left(\frac{g(g+1)}{2}\right)^{\frac{g+1}{2}} \mathrm{e}^{-\frac{\pi y_{g}}{4}}
\end{aligned}
$$

car la fonction $x \longrightarrow x^{g} \exp \left(-\frac{\pi x^{2} y_{g}}{4}\right)$ atteint son maximum en $x=\sqrt{\frac{2 g}{\pi y_{g}}}$ (et modulo une estimation plus précise si $g=1$ ).

On remarque maintenant que $A_{k+1}$ est majoré par le volume de l'ellipsoïde

$$
\mathcal{E}:=\left\{x \in \mathbb{R}^{g} ;{ }^{t} x y x \leq\left(\frac{k+1}{2} \sqrt{y_{g}}+\sqrt{\frac{g(g+1)}{8}} \sqrt{y_{g}}\right)^{2}\right\}
$$

car, d'après le lemme 6.4 , point (ii), une maille fondamentale de $\mathbb{Z}^{g}$ centrée en un point $m+\frac{e_{g}}{2}$ satisfaisant ${ }^{t}\left(2 m+e_{g}\right) y\left(2 m+e_{g}\right) \leq(k+1)^{2} y_{g}$ est contenue dans cet ellipsoïde. Le volume en question est égal à

$$
\frac{\left(\pi y_{g}\right)^{\frac{g}{2}}}{2^{g} \Gamma\left(1+\frac{g}{2}\right) \operatorname{det}(y)^{\frac{1}{2}}} \cdot\left(k+1+\sqrt{\frac{g(g+1)}{2}}\right)^{g},
$$

d'où

$$
A_{k+1} \leq k^{g} \cdot(\mathrm{e} \pi(g+3))^{g / 2} \cdot y_{g}^{g / 2} \operatorname{det}(y)^{-1 / 2},
$$

car

$$
k+1+\sqrt{\frac{g(g+1)}{2}} \leq 2 k\left(1+\sqrt{\frac{g(g+1)}{2}}\right) \quad \text { et } \quad \frac{\left(1+\sqrt{\frac{g(g+1)}{2}}\right)^{g}}{\Gamma\left(1+\frac{g}{2}\right)} \leq(\mathrm{e}(g+3))^{\frac{g}{2}} .
$$

En tenant compte de l'inégalité précédente, on obtient :

$$
\left|\theta_{\left(\frac{e g}{2}, b\right)}(\tau, 0)\right| \leq 4\left(\frac{\mathrm{e} \pi}{2} g(g+1)(g+3)\right)^{\frac{g+1}{2}} y_{g}^{\frac{g}{2}} \operatorname{det}(y)^{-\frac{1}{2}} \exp \left(-\frac{\pi y_{g}}{4}\right) .
$$

En tenant compte du lemme 6.4, point (i), pour obtenir l'inégalité (là encore, il convient d'être plus précis si $g=1$ )

$$
\operatorname{det}(y) \geq\left(\frac{3}{2 g(g-1)}\right)^{\frac{g(g-1)}{2}}\left(\frac{\sqrt{3}}{2}\right)^{g-1} y_{g},
$$


ceci nous donne, vu que $y_{g}^{\frac{g-1}{2}} \leq g^{g} \exp \left(\frac{\pi y_{g}}{8}\right)$ :

$$
\begin{aligned}
\left|\theta_{\left(\frac{e g}{2}, b\right)}(\tau, 0)\right| & \leq 4\left(\frac{2 g(g-1)}{3}\right)^{\frac{g(g-1)}{4}}\left(\frac{\mathrm{e} \pi}{\sqrt{3}} g(g+1)(g+3)\right)^{\frac{g+1}{2}} y_{g^{\frac{g-1}{2}}} \exp \left(-\frac{\pi y_{g}}{4}\right) \\
& \leq(3 g)^{\frac{g^{2}}{2}+g+\frac{3}{2}} y_{g}^{\frac{g-1}{2}} \exp \left(-\frac{\pi y_{g}}{4}\right) \\
& \leq(4 g)^{2 g^{2}} \exp \left(-\frac{\pi y_{g}}{8}\right) .
\end{aligned}
$$

Le lemme 6.6 est donc établi ${ }^{15}$

Nous pouvons maintenant passer au lemme «matriciel » de MAssER. Pour tout élément $\tau$ de $\mathfrak{S}_{g}$, nous noterons $\boldsymbol{k}_{\tau}$ le corps $\mathbb{Q}\left[\theta_{p}(\tau, 0)\right]_{p \in \mathcal{Z}_{2}^{2}}$.

Lemme 6.7. Soit $\tau$ un élément de $\mathfrak{S}_{g}$ tel que $\boldsymbol{k}_{\tau}$ soit contenu dans un corps de nombres $\boldsymbol{k}$, de degré d sur $\mathbb{Q}$. Soit $\sigma$ un plongement complexe de $\boldsymbol{k}$ et $\tau(\sigma)$ un élément de $\mathfrak{S}_{g}$ satisfaisant $A_{\tau}^{\sigma}=A_{\tau(\sigma)}$. Soit enfin $\tau^{\prime}(\sigma)$ un représentant de $\tau(\sigma)$ dans $\mathfrak{F}_{g}$ et $y^{\prime}(\sigma)$ la partie imaginaire de $\tau^{\prime}(\sigma)$. On a alors :

$$
\frac{1}{d} \sum_{\sigma}\left\|y^{\prime}(\sigma)\right\| \leq \frac{8}{\pi} \cdot\left(\max \{1 ; h(A)\}+2 g^{2} \log (4 g)\right) .
$$

Démonstration. (Voir aussi [Ma-Wu2], lemme 8-6.) Quitte à faire une extension de $\boldsymbol{k}$, on peut supposer que $\tau^{\prime}(\sigma)=\tau(\sigma)$; c'est ce que nous ferons ; soit donc $\sigma$ un plongement complexe de $\boldsymbol{k}$. Choisissons un élément $p(\sigma) \in \mathcal{Z}_{2}^{2}$ tel que $\left|\theta_{p(\sigma)}(\tau(\sigma), 0)\right|$ soit maximal. De même, en tenant compte du lemme 6.6, choisissons un élément $b \in \mathcal{Z}_{2}$ tel que $0 \neq\left|\theta_{\left(\frac{e_{g}}{2}, b\right)}(\tau(\sigma), 0)\right| \leq(4 g)^{g^{2}} \exp \left(-\frac{\pi}{8}\left\|y^{\prime}(\sigma)\right\|\right)$. La définition de la hauteur de WEIL nous assure alors que :

$$
\begin{aligned}
\frac{1}{d} \sum_{\sigma} \log \left|\frac{\theta_{\left(\frac{e_{g}}{2}, b\right)}(\tau(\sigma), 0)}{\theta_{p(\sigma)}(\tau(\sigma), 0)}\right| & \geq-\frac{1}{d} \sum_{\sigma} \log \max _{p \in \mathcal{Z}_{2}^{2}}\left\{\left|\frac{\theta_{p}(\tau(\sigma), 0)}{\theta_{\left(\frac{e_{g}}{2}, b\right)}(\tau(\sigma), 0)}\right|\right\} \\
& \geq-h_{W, \mathcal{L} \otimes 16}\left(A_{\tau}\right) \\
& \geq-h_{\mathcal{L} \otimes 16}\left(A_{\tau}\right) .
\end{aligned}
$$

Par ailleurs, en tenant compte des lemmes 6.5 et 6.6 :

$$
\frac{1}{d} \sum_{\sigma} \log \left|\frac{\theta_{\left(\frac{e_{g}}{2}, b\right)}(\tau(\sigma), 0)}{\theta_{p(\sigma)}(\tau(\sigma), 0)}\right| \leq-\frac{\pi}{8 d} \sum_{\sigma}\left\|y^{\prime}(\sigma)\right\|+g^{2} \log (4 g),
$$

et le lemme 6.7 est établi.

\footnotetext{
15 On peut remarquer que cette majoration est un peu brutale en $y_{g}$; la valeur exacte du terme asymptotique dominant est bien entendu $\exp \left(-\frac{\pi y_{g}}{4}\right)$.
} 
Concluons cet appendice par une estimation du rayon d'injectivité $\mathcal{R}_{\text {inj }}$ apparaissant dans les estimations précédentes.

Lemme 6.8. Avec les notations introduites précédemment on a:

$$
\mathcal{R}_{\text {inj }} \geqslant \sqrt{\frac{32 \pi}{(g+1) \max _{v}\left\{\left\|\operatorname{Im} \tau_{v}\right\|\right\}}}
$$

où le maximum porte sur toutes les places archimédiennes d'un corps de définition de la variété abelienne A.

En particulier,

$$
\mathcal{R}_{\text {inj }} \geq \frac{1}{g^{2}} \cdot(d \cdot \max \{1 ; h(A)\})^{-1 / 2},
$$

où d est le degré sur $\mathbb{Q}$ d'un corps de définition $\boldsymbol{k}$ de $A$.

Démonstration. Fixons une place archimédienne $v$ d'un corps de définition $\boldsymbol{k}$ de $A$, et posons $\tau_{v}=\tau=x+\mathrm{i} y$ et $\lambda=a+(x+\mathrm{i} y) b$, où $a, b \in \mathbb{Z}^{g}$ et $x, y \in \mathrm{M}_{g}(\mathbb{R})$. Avec ces notations,

$$
\begin{aligned}
\min _{\lambda \in \Lambda \backslash\{0\}}\{H(\lambda, \lambda)\} & \geq 16 \min _{(a, b) \in \mathbb{Z}^{2 g} \backslash\{0\}}\left\{{ }^{t}(a+x b) y^{-1}(a+x b)+{ }^{t} b y b\right\} \\
& \geq 16 \min \left\{\min _{b \in \mathbb{Z}^{g} \backslash\{0\}}\left\{{ }^{t} b y b\right\} ; \min _{a \in \mathbb{Z}^{g} \backslash\{0\}}\left\{{ }^{t} a y^{-1} a\right\}\right\} \\
& \geq 16 \min \left\{\frac{\sqrt{3}}{2} ; \frac{1}{\lambda_{\max }}\right\}
\end{aligned}
$$

où $\lambda_{\max }$ est la plus grande valeur propre de $y$.

Mais, par le lemme 6.4, point (ii), si $\xi$ est un vecteur propre de $y$ associé à $\lambda_{\max }$, on a :

$$
\frac{g+1}{2} y_{g}\|\xi\|^{2} \geq \frac{g+1}{2} \sum_{i=1}^{g} y_{i} \xi_{i}^{2} \geq{ }^{t} \xi y \xi=\lambda_{\max }\|\xi\|^{2}
$$

D'où,

$$
\frac{1}{\lambda_{\max }} \geq \frac{2}{(g+1)\|y\|}
$$

en reportant ces inégalités dans la définition de $\mathcal{R}_{\text {inj }}$, on en déduit bien la première partie du lemme 6.8. Pour le supplément, il suffit de combiner la première inégalité avec le lemme 6.7. Le lemme 6.8 est donc entièrement établi.

On notera que si l'on tient compte du théorème 1.1 de [Bo-Da], on déduit immédiatement du lemme 6.7 le corollaire suivant :

Corollaire 6.9. Soit $A$ une variété abelienne de dimension g, principalement polarisée, définie sur un corps de nombres $\boldsymbol{k}$ sur lequel elle admet une réduction 
semi-stable, et soit $h_{F}(A)$ sa hauteur de Faltings. Alors :

$$
\left|h(A)-\frac{1}{2} h_{F}(A)\right| \leq \frac{1}{4} g \log (\max \{1 ; h(A)\})+\frac{1}{4}(g+1)^{4} .
$$

\section{Références}

[Bi-La] Ch. Birkenhake et H. Lange, Complex abelian varieties, Grundlehren Math. Wiss. t. 302, Springer-Verlag, Berlin-Heidelberg-New-York, 1992.

[Bo] J.-B. Bost, Périodes et isogénies des variétés abéliennes sur les corps de nombres (d'après D. Masser et G. Wüstholz), In Séminaire Bourbaki 94/95, Astérisque 237 (1996), 115-161.

[Bo-Da] J.-B. Bost et S. David, Notes on the comparison of heights of abelian varieties, Manuscrit, 1999.

[Ca-Ha-Ma] L. Caporaso, J. Harris et B. Mazur, Uniformity of rational points, J. Amer. Math. Soc. 10 (1997), 1-35.

[Da] S. David, Fonctions thêta et points de torsion des variétés abéliennes, Compositio Math. 78 (1991), 121-160.

[Da-Phi1] S. David et P. Philippon, Minorations des hauteurs normalisées des sous-variétés de variétés abeliennes, In Number Theory, Tiruchirapalli, India, 3-6 Janvier 1996, V. K. Murty et M. Waldschmidt, éditeurs, Contemp. Math. 210 (1998), 333-364.

[Da-Phi2] S. David et P. Philippon, Minorations des hauteurs normalisées des sous-variétés des tores, Ann. Scuola Norm. Sup. Pisa Cl. Sci. (4) 28 (1999), 489-543.

[Ev] J.-H. Evertse, Points on subvarieties of tori, Rep. Math. Inst. Univ. Leiden, 2000.

[Gr-Ha] P. Griffiths et J. Harris, Principles of algebraic geometry, Wiley-Interscience, 1978.

[Ig] J. I. Igusa, Theta functions, Grundlehren Math. Wiss. 194, Springer-Verlag, BerlinHeidelberg-New-York, 1972.

[La-Ru] H. Lange et W. Ruppert, Complete systems of addition laws on abelian varieties, Invent. Math. 79 (1985), 603-610.

[Ma-Za] Y. Manin et Y. Zarhin, Heights on families of abelian varieties, Mat. Sb. 89 (1972), 171-181; traduit du texte russe Math. USSR Sb. 18 (1972), 169-179.

[Ma] D. W. Masser, Small values of heights on families of abelian varieties, In Diophantine approximation and transcendence theory (Bonn, 1985), G. Wüstholz, éditeur, Lecture Notes in Math. 1290 (1987), 109-148.

[Ma-Wu2] D. W. Masser et G. Wüstholz, Periods and minimal abelian subvarieties, Ann. of Math. 137 (1993), 407-458.

[Mu] D. Mumford, On the equations defining abelian varieties I, Invent. Math. 1 (1966), $287-354$.

[Ph] P. Philippon, Sur des hauteurs alternatives I ; II ; III, Math. Ann. 289 (1991), 255283 ; Ann. Inst. Fourier (Grenoble) 44 (4) (1994), 1043-1065; J. Math. Pures Appl. 74 (4) (1995), 345-365.

[Ré] G. Rémond, Décompte dans une conjecture de Lang, Invent. Math. 142 (3) (2000), $513-545$.

[Schm] W. M. Schmidt, Heights of points on subvarieties of $\mathbb{G}_{m}^{n}$, Number Theory 93-94, S. David éditeur, London Math. Soc. Ser. 235, Cambridge University Press, 1996. 
[Sz-Ul-Zh] L. Szpiro, E. Ullmo et S. Zhang, Équirépartition des petits points, Invent. Math. 127 (1997), 337-347.

[Ul] E. Ullmo, Positivité et discrétion des points algébriques des courbes, Ann. of Math. 147 (1996), 81-95.

[Wa] M. Waldschmidt, Transcendance et exponentielles en plusieurs variables, Invent. Math. 63 (1981), 97-127.

[Zh1] S. Zhang, Small points and adelic metrics, J. Algebraic Geom. 4 (1995), 281-300.

[Zh2] S. Zhang, Equidistribution of small points on abelian varieties, Ann. of Math. 147 (1996), 159-165.

[Zi] H. Zimmer, On the difference of the Weil height and the Néron-Tate height, Math. Z. 147 (1976), 35-51.

Sinnou David et Patrice Philippon

Universités de Paris VI et VII

Institut de Mathématiques de Jussieu

UMR 7586 du C. N. R. S. - UFR 921

4, Place Jussieu

75252 Paris cedex 05

France

e-mail: david@math.jussieu.fr

e-mail: pph@math.jussieu.fr

(Received: February 2, 2001)

(10) To access this journal online:

(4) http://www.birkhauser.ch 